$\mathrm{IPPP} / 04 / 70$

$\mathrm{DCPT} / 04 / 140$

$\mathrm{SINP} / \mathrm{TNP} / 04-18$

TIFR/TH/04-29

hep-ph/0411018

\title{
Next-to-Leading Order QCD Corrections to the Drell-Yan Cross Section in Models of TeV-Scale Gravity
}

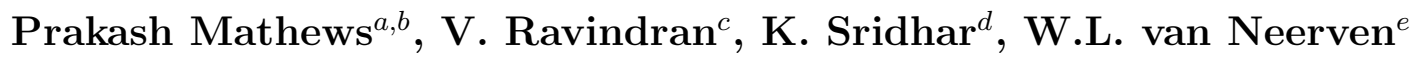

a) Institute for Particle Physics Phenomenology, University of Durham, Durham DH1 3LE, UK

b) Theory Group, Saha Institute of Nuclear Physics, 1/AF Bidhan Nagar, Kolkata 700 064, India

c) Harish-Chandra Research Institute, Chhatnag Road, Jhunsi, Allahabad, India.

d) Department of Theoretical Physics, Tata Institute of Fundamental Research, Homi Bhabha Road, Mumbai 400 005, India.

e)Instituut-Lorentz, University of Leiden, PO Box 9506, 2300 RA Leiden, The Netherlands.

\begin{abstract}
The first results on next-to-leading order QCD corrections to graviton-induced processes in hadron collisions in models of $\mathrm{TeV}$-scale gravity are presented focusing on the case of dilepton pair production in $\bar{p} p$ and $p p$ collisions. Distributions in the invariant mass $Q$, the longitudinal fraction $x_{F}$, the rapidity $Y$ and the forward-backward asymmetry of the lepton pair are studied. The quantitative impact of the QCD corrections for searches of extra dimensions at hadron colliders is investigated. It turns out that at the LHC $(\sqrt{S}=14 \mathrm{TeV})$ the $K$-factor is rather large $(K=1.6)$ for large invariant mass $Q$ of the lepton pair, indicating the importance of accounting for these QCD corrections in the experimental search for $\mathrm{TeV}$-scale gravity. At the Tevatron, the K-factor does not substantially deviate from the Standard Model value. However, its inclusion is necessitated to make the cross-section stable with respect to scale variations.
\end{abstract}

PACS numbers: 11.10.Kk, 12.10.-g, 12.38.-t, 13.60.Hb. 


\section{Introduction}

In the recent past, models of extra dimensions have been studied as serious contenders for physics beyond the Standard Model. These extra-dimensional models are attractive because they help address the hierarchy between the electroweak and the Planck scales. In the model of Arkani-Hamed, Dimopoulos and Dvali (the ADD model) [1] the Planck scale is reduced to a $\mathrm{TeV}$ and the hierarchy problem thereby avoided by invoking a large magnitude of $d$ extra dimensions.

In the ADD model, the Standard Model (SM) fields are constrained to a 3-brane, while gravitons propagate in the $4+d$ dimensions. Then the size of the extra dimensions is only constrained by the length scales to which the gravitational inverse square law has been experimentally tested, which are currently probing the sub-millimetre range. For $d$ between 2 and 6 , the size of the extra dimensions varies from a millimetre to a Fermi. The relation between the 4-dimensional Planck scale $M_{P}$ and the scale $M_{S}$ in $(4+d)$-dimension is

$$
M_{P} \approx M_{S}^{(d+2)} R^{d}
$$

where $R$ is the compactification radius. In the ADD model, because $R$ is large it is possible to lower $M_{S}$ down to a $\mathrm{TeV}$ and avoid the hierarchy problem. An important consequence of the lowering of the Planck scale is that quantum gravity effects could be tested at energies of $\mathcal{O}(\mathrm{TeV})$. So it opens up a new area of studies on the effects of gravitons at present and future colliders.

The $4+d$ dimensional graviton manifests in 4-dimensions as a tower of massive Kaluza-Klein $(\mathrm{KK})$ modes. These KK modes interact with the SM particles confined to the 3-brane via the energy-momentum tensor. Each KK mode couples to the SM particles with a coupling of the order of $1 / M_{P}$, (we will use $\kappa$ in the rest of the paper). As can be seen the coupling of each KK mode to the SM particles are highly suppressed. But the effective coupling is obtained after summing over all the KK modes and due to the high multiplicity of the KK modes the effective interaction has a strength of $1 / M_{S}$ $[2,3]$. This enhanced coupling provides viable signatures of the graviton KK modes at colliders. Both real graviton production and the effects of virtual gravitons in various processes have been studied in the literature [4] and have yielded bounds on $M_{S}$ which are in the ball-park of a $\mathrm{TeV}$.

These collider processes involving gravitons have, however, been studied at the leading order (LO) in QCD ${ }^{1}$. At hadron colliders such as present Tevatron and upcoming Large Hadron Collider (LHC), the theoretical uncertainties coming from the QCD effects due to initial state partons are quite sizable. The sources of these uncertainties are two fold: renormalisation and factorisation scales and the parton distribution functions. The scale uncertainties can come from the strong coupling constant as well as parton distribution functions. Experience with next to leading order (NLO)

\footnotetext{
${ }^{1}$ Recently NLO-QCD corrections to $e^{+} e^{-} \rightarrow$ hadrons have been studied in [5]
} 
contributions to SM processes strongly suggests that the LO corrections at hadronic colliders are quiet unreliable [6, 7]. The standard Drell-Yan (DY) processes and Higgs production at hadron colliders, for example, not only get large corrections but the theoretical uncertainties get reduced significantly from NLO corrections.

It is with this motivation that the present paper presents the results of the computation of NLO-QCD corrections to the DY process, $P_{1}+P_{2} \rightarrow \mu^{+} \mu^{-}+X$, where $P_{1}$, $P_{2}$ are initial hadrons and $X$ is an arbitrary hadronic final state. This process takes place via the exchange of $\gamma, Z$ and graviton. These NLO results are used to study the impact of the QCD correction for this process at the present Tevatron collider and the future LHC which is planned to operate at centre-of-mass energies around $14 \mathrm{TeV}$. This process had been considered earlier to LO $[8,9]$ in the ADD model.

The results we present here are for the ADD model, but since the QCD corrections, presented here, are model-independent they may equally be used for studying the Randall-Sundrum model of warped compactification [10].

This paper is organised as follows. In Sec. 2 we describe additional graviton vertices needed to perform the NLO QCD corrections. We study the distributions in the invariant mass $Q$ (Sec. 3), the longitudinal fraction $x_{F}$ (Sec. 4), the rapidity $Y$ (Sec. 5) and the forward-backward asymmetry (Sec. 6) of lepton pair. Finally in Sec. 7 we present the discussion and summarise the results. In Appendix A and B we present the detailed expressions needed for $x_{F}$ and $Y$ distributions in Sec. 4 and Sec. 5 respectively. 


\section{The Model}

We work with the following action

$$
\mathcal{S}=\mathcal{S}_{S M}-\frac{\kappa}{2} \int d^{4} x \Theta_{\mu \nu}^{Q C D}(x) h^{\mu \nu}(x)
$$

where $S_{S M}$ is the Standard Model action, $h^{\mu \nu}$ is the graviton field and $\kappa$ is the strength of the interaction. The energy momentum tensor in QCD is given by

$$
\begin{aligned}
\Theta_{\mu \nu}^{Q C D}= & -g_{\mu \nu} \mathcal{L}_{Q C D}-F_{\mu \rho}^{a} F_{\nu}^{a \rho}-\frac{1}{\xi} g_{\mu \nu} \partial^{\rho}\left(A_{\rho}^{a} \partial^{\sigma} A_{\sigma}^{a}\right) \\
& +\frac{1}{\xi}\left(A_{\nu}^{a} \partial_{\mu}\left(\partial^{\sigma} A_{\sigma}^{a}\right)+A_{\mu}^{a} \partial_{\nu}\left(\partial^{\sigma} A_{\sigma}^{a}\right)\right)+\frac{i}{4}\left[\bar{\psi} \gamma_{\mu}\left(\vec{\partial}_{\nu}-i g T^{a} A_{\nu}^{a}\right) \psi\right. \\
& -\bar{\psi}\left(\overleftarrow{\partial}_{\nu}+i g T^{a} A_{\nu}^{a}\right) \gamma_{\mu} \psi+\bar{\psi} \gamma_{\nu}\left(\vec{\partial}_{\mu}-i g T^{a} A_{\mu}^{a}\right) \psi \\
& \left.-\bar{\psi}\left(\overleftarrow{\partial}_{\mu}+i g T^{a} A_{\mu}^{a}\right) \gamma_{\nu} \psi\right]+\partial_{\mu} \bar{\omega}^{a}\left(\partial_{\nu} \omega^{a}-g f^{a b c} A_{\nu}^{c} \omega^{b}\right) \\
& +\partial_{\nu} \bar{\omega}^{a}\left(\partial_{\mu} \omega^{a}-g f^{a b c} A_{\mu}^{c} \omega^{b}\right) .
\end{aligned}
$$

In the above equation, $\xi$ is the gauge fixing parameter. We work in the Feynman gauge in which the gauge parameter $\xi=1$. We have displayed explicitly the ghost terms with the ghost fields $\omega^{a}(x)$ since they contribute to our one-loop virtual corrections to the process under study. The presence of the ghost fields introduces two new vertices (see Fig. 1).

1) graviton-ghost-ghost vertex
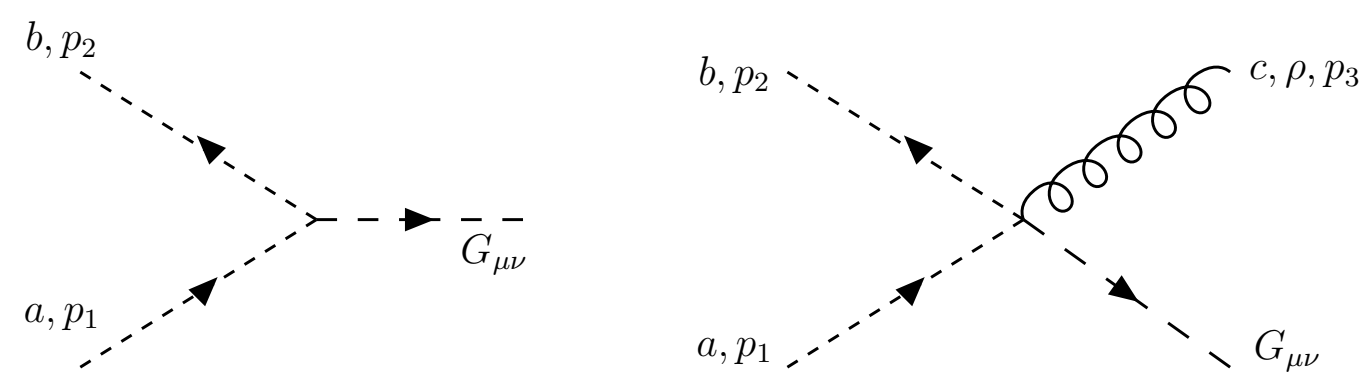

Figure 1: Ghost vertices

$$
\Gamma_{\mu \nu}\left(p_{1}, p_{2}\right)=-i \frac{\kappa}{2} \delta^{a b} C_{\mu \nu, \rho \sigma} p_{1}^{\rho} p_{2}^{\sigma}
$$


2) graviton-ghost-ghost-gluon vertex

$$
\Gamma_{\mu \nu, \rho}\left(p_{1}, p_{2}\right)=\frac{\kappa}{2} g f^{a b c} C_{\mu \nu, \rho \sigma} p_{2}^{\sigma}
$$

where

$$
C_{\mu \nu, \rho \sigma}=g_{\mu \rho} g_{\nu \sigma}+g_{\mu \sigma} g_{\nu \rho}-g_{\mu \nu} g_{\rho \sigma}
$$

The $\mu, \nu$ indices refer to the graviton. The momenta $p_{1}, p_{2}$ and the colour indices $a, b$ correspond to the incoming and outgoing ghosts respectively. Finally $c, \rho, p_{3}$ indicate the colour index, Lorentz index and momentum of the gluon. The other Feynman rules are given in Ref. [3]. ${ }^{2}$

\footnotetext{
${ }^{2}$ The only exceptions are the Feynman rules for the fermion-anti-fermion-gauge boson-graviton vertex and the three gauge boson-graviton vertex which differ from those of Ref. [3] by an overall sign.
} 


\section{Invariant lepton pair mass distribution $d \sigma / d Q^{2}$}

We start by considering $P_{1}, P_{2}$ scattering to leptonic final states, say $\mu^{+}, \mu^{-}$

$$
P_{1}\left(p_{1}\right)+P_{2}\left(p_{2}\right) \rightarrow \mu^{+}\left(l_{1}\right)+\mu^{-}\left(l_{2}\right)+X\left(P_{X}\right),
$$

where $p_{1}, p_{2}$ are the momenta of incoming hadrons $P_{1}$ and $P_{2}$ respectively and $\mu^{-}, \mu^{+}$ are the outgoing leptons which have the momenta $l_{1}, l_{2}$. The final inclusive hadronic state is denoted by $X$ and carries the momentum $P_{X}$. In the QCD improved parton model, the hadronic cross section can be expressed in terms of partonic cross sections convoluted with appropriate parton distribution functions as follows

$$
\begin{aligned}
2 S \frac{d \sigma^{P_{1} P_{2}}}{d Q^{2}}\left(\tau, Q^{2}\right)= & \sum_{a b=q, \bar{q}, g} \int_{0}^{1} d x_{1} \int_{0}^{1} d x_{2} f_{a}^{P_{1}}\left(x_{1}\right) f_{b}^{P_{2}}\left(x_{2}\right) \\
& \times \int_{0}^{1} d z 2 \hat{s} \frac{d \hat{\sigma}^{a b}}{d Q^{2}}\left(z, Q^{2}\right) \delta\left(\tau-z x_{1} x_{2}\right) .
\end{aligned}
$$

The scaling variables are defined by $k_{1}=x_{1} p_{1}, k_{2}=x_{2} p_{2}$ where $k_{1}, k_{2}$ are the momenta of incoming partons.

$$
\begin{aligned}
&\left(p_{1}+p_{2}\right)^{2} \equiv S, \quad\left(k_{1}+k_{2}\right)^{2} \equiv \hat{s}, \quad\left(l_{1}+l_{2}\right)^{2}=q \cdot q \equiv Q^{2}, \\
& \tau=\frac{Q^{2}}{S}, \quad z=\frac{Q^{2}}{\hat{s}}, \quad \tau=x_{1} x_{2} z .
\end{aligned}
$$

The partonic cross section for the process $a\left(k_{1}\right)+b\left(k_{2}\right) \rightarrow j(-q)+\sum_{i}^{m} X_{i}\left(-p_{i}\right)$ is given by

$$
2 \hat{s} \frac{d \hat{\sigma}^{a b}}{d Q^{2}}=\frac{1}{2 \pi} \sum_{j j^{\prime}=\gamma, Z, G} \int d P S_{m+1}\left|M^{a b \rightarrow j j^{\prime}}\right|^{2} \cdot P_{j}(q) \cdot P_{j^{\prime}}^{*}(q) \cdot \mathcal{L}^{j j^{\prime} \rightarrow l^{+} l^{-}}(q) .
$$

In the above equation, the sum over Lorentz indices between matrix element squared and the propagators is implicit through a symbol "dot product". The $m+1$ body phase space is defined as

$$
\begin{aligned}
\int d P S_{m+1}= & \int \prod_{i}^{m}\left(\frac{d^{n} p_{i}}{(2 \pi)^{n}} 2 \pi \delta^{+}\left(p_{i}^{2}\right)\right) \frac{d^{n} q}{(2 \pi)^{n}} 2 \pi \delta^{+}\left(q^{2}-Q^{2}\right) \\
& \times(2 \pi)^{n} \delta^{(n)}\left(k_{1}+k_{2}+q+\sum_{i}^{m} p_{i}\right),
\end{aligned}
$$


where $n$ is the space-time dimension. The propagators are

$$
\begin{gathered}
P_{\gamma}(q)=-\frac{i}{Q^{2}} g_{\mu \nu} \equiv g_{\mu \nu} \tilde{P}_{\gamma}\left(Q^{2}\right) \\
P_{Z}(q)=-\frac{i}{\left(Q^{2}-M_{Z}^{2}-i M_{Z} \Gamma_{Z}\right)} g_{\mu \nu} \equiv g_{\mu \nu} \tilde{P}_{Z}\left(Q^{2}\right) \\
P_{G}(q)=\mathcal{D}\left(Q^{2}\right) B_{\mu \nu \lambda \rho}(q) \equiv B_{\mu \nu \lambda \rho}(q) \tilde{P}_{G}\left(Q^{2}\right)
\end{gathered}
$$

where

$$
\begin{aligned}
B_{\mu \nu \rho \sigma}(q) & =\eta_{\mu \rho} \eta_{\nu \sigma}+\eta_{\mu \sigma} \eta_{\nu \rho}-\frac{2}{n-1} \eta_{\mu \nu} \eta_{\rho \sigma} \\
\eta_{\mu \nu}(q) & =-g_{\mu \nu}+\frac{q_{\mu} q_{\nu}}{Q^{2}} .
\end{aligned}
$$

The summation of the virtual KK modes in the time-like propagators [3] leads to

$$
\mathcal{D}\left(Q^{2}\right)=\frac{Q^{d-2} R^{d}}{\Gamma(d / 2)(4 \pi)^{d / 2}} 2 I\left(\frac{M_{S}}{Q}\right),
$$

where the integral $I$ is regulated by an ultraviolet cutoff, presumably of the order of $M_{S}[2,3]$, which sets the limit on the applicability of the effective theory. For the DY case under consideration this consistency would imply $Q<M_{S}$. Further relating the gravitational coupling, the volume of extra dimension and the cutoff scale [3]

$$
\kappa^{2} R^{d}=8 \pi(4 \pi)^{d / 2} \Gamma(d / 2) M_{S}^{-(d+2)},
$$

we express the function $\mathcal{D}\left(Q^{2}\right)$ as

$$
\mathcal{D}\left(Q^{2}\right)=16 \pi\left(\frac{Q^{d-2}}{\kappa^{2} M_{S}^{d+2}}\right) I\left(\frac{M_{S}}{Q}\right) .
$$

The summation over the non-resonant KK modes yields

$$
\begin{array}{ll}
I(\omega)=-\sum_{k=1}^{d / 2-1} \frac{1}{2 k} \omega^{2 k}-\frac{1}{2} \log \left(\omega^{2}-1\right), & d=\text { even }, \\
I(\omega)=-\sum_{k=1}^{(d-1) / 2} \frac{1}{2 k-1} \omega^{2 k-1}+\frac{1}{2} \log \left(\frac{\omega+1}{\omega-1}\right), & d=\text { odd },
\end{array}
$$


where $\omega=M_{S} / Q$. The leptonic tensor involves the computation of square of the matrix element for the process $\gamma / Z / G \rightarrow \mu^{+}+\mu^{-}$and the 2-body phase space integral

$$
\mathcal{L}^{j j^{\prime} \rightarrow \mu^{+} \mu^{-}}(q)=\int \prod_{i=1}^{2}\left(\frac{d^{n} l_{i}}{(2 \pi)^{n}} 2 \pi \delta^{+}\left(l_{i}^{2}\right)\right)(2 \pi)^{n} \delta^{(n)}\left(q-l_{1}-l_{2}\right)\left|M^{j j^{\prime} \rightarrow \mu^{+} \mu^{-}}\right|^{2} .
$$

The leptonic part is easy to compute. It is equal to

$$
\begin{aligned}
\mathcal{L}^{j j^{\prime} \rightarrow \mu^{+} \mu^{-}}(q) & =\eta_{\mu \nu}(q) L_{j j^{\prime}}\left(Q^{2}\right), \quad j j^{\prime}=\{\gamma \gamma, Z Z, \gamma Z\}, \\
\mathcal{L}^{G G \rightarrow \mu^{+} \mu^{-}}(q) & =B_{\mu \nu \rho \sigma}(q) L_{G}\left(Q^{2}\right),
\end{aligned}
$$

with

$$
\begin{array}{rlrl}
L_{\gamma \gamma}\left(Q^{2}\right) & =Q^{2} \frac{2 \alpha}{3}, & L_{\gamma Z}\left(Q^{2}\right)=-Q^{2} \frac{2 \alpha g_{e}^{V}}{3 c_{w} s_{w}} \\
L_{Z Z}\left(Q^{2}\right)=Q^{2} \frac{2 \alpha}{3 c_{w}^{2} s_{w}^{2}}\left(\left(g_{e}^{V}\right)^{2}+\left(g_{e}^{A}\right)^{2}\right), & L_{G G}\left(Q^{2}\right)=Q^{4} \frac{\kappa^{2}}{640 \pi},
\end{array}
$$

where $\alpha$ is the fine structure constant

$$
\begin{array}{lll}
c_{w}=\cos \theta_{W}, & s_{w}=\sin \theta_{W}, \\
g_{a}^{V}=\frac{1}{2} T_{a}^{3}-s_{w}^{2} Q_{a}, & g_{a}^{A}=-\frac{1}{2} T_{a}^{3},
\end{array}
$$

and $Q_{a}$ is the electric charge of quarks and leptons. Hence, we obtain

$$
2 S \frac{d \sigma^{P_{1} P_{2}}}{d Q^{2}}\left(\tau, Q^{2}\right)=\frac{1}{2 \pi} \sum_{j j^{\prime}=\gamma, Z, G} \tilde{P}_{j}\left(Q^{2}\right) \tilde{P}_{j^{\prime}}^{*}\left(Q^{2}\right) L_{j j^{\prime}}\left(Q^{2}\right) W_{j j^{\prime}}^{P_{1} P_{2}}\left(\tau, Q^{2}\right) .
$$

The hadronic structure function is equal to

$$
\begin{aligned}
W_{j j^{\prime}}^{P_{1} P_{2}}\left(\tau, Q^{2}\right)= & \sum_{a b, j j^{\prime}} \int d x_{1} \int d x_{2} f_{a}^{P_{1}}\left(x_{1}\right) f_{b}^{P_{2}}\left(x_{2}\right) \\
& \times \int d z \delta\left(\tau-z x_{1} x_{2}\right) \int d P S_{m+1}\left|M^{a b \rightarrow j j^{\prime}}\right|^{2} T_{j j^{\prime}}(q)
\end{aligned}
$$

with

$$
\begin{aligned}
T_{j j^{\prime}} & =\eta_{\mu \nu}(q), \quad j j^{\prime}=\gamma \gamma, \gamma Z, Z Z, \\
T_{G G} & =B_{\mu \nu \rho \sigma}(q) .
\end{aligned}
$$



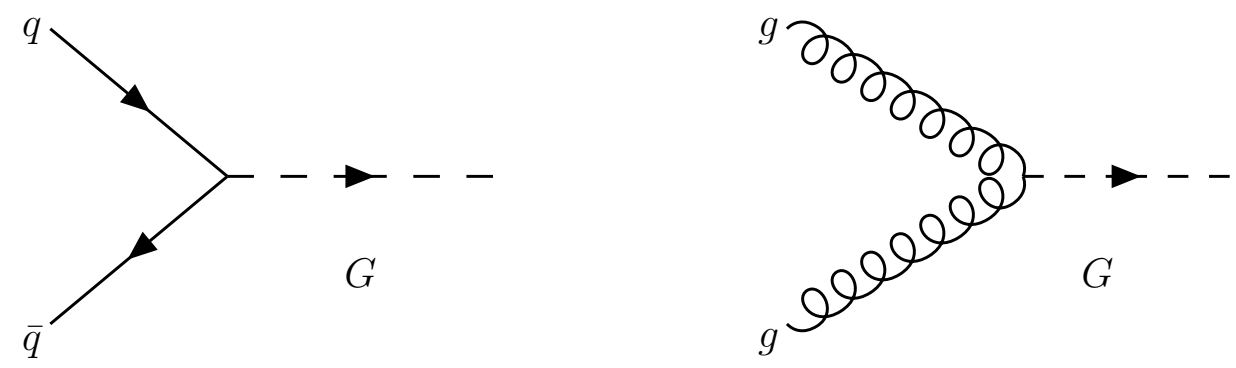

Figure 2: Born contributions:

The tensors $\eta_{\mu \nu}(q)$ and $B_{\mu \nu \rho \sigma}(q)$ are defined in Eq. (3.8). To compute the $Q^{2}$ distribution of the di-lepton pairs, the matrix element squared $\left|M^{a b \rightarrow j j^{\prime}}\right|^{2} T_{j j^{\prime}}(q)$ has to be substituted in Eq.(3.19) provided the integrations over $d P S_{m+1}$ and $d z$ are performed in a suitable frame. We define the bare partonic coefficient function $\bar{\Delta}_{a b}^{j j^{\prime}}\left(z, Q^{2}\right)$ as

$$
\bar{\Delta}_{a b}^{j j^{\prime}}\left(z, Q^{2}\right)=C_{j j^{\prime}} \int d P S_{m+1}\left|M^{a b \rightarrow j j^{\prime}}\right|^{2} T_{j j^{\prime}}(q)
$$

where

$$
\begin{array}{rlrl}
C_{j j^{\prime}} & =\frac{1}{e^{2}} & & j j^{\prime}=\gamma \gamma, Z Z, \gamma Z, \\
& =\frac{1}{Q^{2} \kappa^{2}} & j j^{\prime}=G G .
\end{array}
$$

There are two classes of processes that contribute to the partonic cross section. The first one has only a virtual photon or a $\mathrm{Z}$ boson whereas the second one only contains a graviton in the intermediate state. Interestingly, the interference between first and second class diagrams identically vanish when the phase space integration is performed. In the case of the photon exchange process, it is easy to understand if we realise that there is no third rank tensor, say $S^{\mu \nu \rho}$ which can be constructed out of $g_{\mu \nu}$ and $q_{\rho}$ $(q . q \neq 0)$ (which are the only tensor and vector available in our problem) that satisfies $q_{\mu} S^{\mu \nu \rho}=0$ (as it should be for the theory where we have gravity coupled to a conserved energy momentum tensor). We found a similar argument for the Levi-Civita tensor which shows up in the case of the electro-weak vertices. Therefore also here there are no interference terms. We could also verify this by explicit computation. At LO the following process contribute when a photon or Z-boson appears in the intermediate state

$$
q+\bar{q} \rightarrow \gamma^{*} / Z^{*}
$$

In the case the Graviton appears in the intermediate state, we have two processes at LO (see Fig. 2)

$$
q+\bar{q} \rightarrow G^{*}, \quad g+g \rightarrow G^{*}
$$


In NLO for the photon or Z-boson we have the following $2 \rightarrow 2$ reactions $[6,7,11]$

$$
\begin{array}{ll}
q+\bar{q} \rightarrow \gamma^{*} / Z^{*}+q, & q+\bar{q} \rightarrow \gamma^{*} / Z^{*}+\text { one loop }, \\
q+g \rightarrow \gamma^{*} / Z^{*}+q, & \bar{q}+g \rightarrow \gamma^{*} / Z^{*}+\bar{q},
\end{array}
$$

and for the graviton we have (see Figs. 3-9)

$$
\begin{array}{ll}
q+\bar{q} \rightarrow G+q, & q+\bar{q} \rightarrow G+\text { one loop }, \\
q+g \rightarrow G+q, & \bar{q}+g \rightarrow G+\bar{q}, \\
g+g \rightarrow G+g, & g+g \rightarrow G+\text { one loop } .
\end{array}
$$

The cross sections beyond leading order involve the computation of one loop virtual gluon corrections and real gluon bremsstrahlung contributions to leading order processes. We also include processes with a gluon in the initial state. Since we are dealing with energy momentum tensor coupled to gravity expressed in terms of renormalised fields and masses, there is no overall ultraviolet renormalisation required. In other words, the operator renormalisation constant for the energy momentum operator is identical to unity to all orders in perturbation theory. But we encounter soft and collinear divergences in our computation. We have used $n$-dimensional regularisation to regulate both these divergences. To that order, we have defined $n=4+\varepsilon$ where $n$ is the space-time dimension. With this procedure all divergences appear as $1 / \varepsilon^{\alpha}$ where $\alpha=1,2$. The soft divergences coming from virtual gluons and bremsstrahlung contributions cancel exactly according to the Bloch-Nordsieck theorem. The remaining collinear divergences are removed by mass factorisation which in our paper is performed in $\overline{M S}$ scheme. The Drell-Yan coefficient function after mass factorisation, denoted by $\Delta_{a b}^{i}\left(z, Q^{2}, \mu_{F}^{2}\right)$, is computed by

$$
\bar{\Delta}_{a b}^{i}\left(z, Q^{2}, 1 / \varepsilon\right)=\sum_{c, d} \Gamma_{c a}\left(z, \mu_{F}^{2}, 1 / \varepsilon\right) \otimes \Gamma_{d b}\left(z, \mu_{F}^{2}, 1 / \varepsilon\right) \otimes \Delta_{c d}^{i}\left(z, Q^{2}, \mu_{F}^{2}\right),
$$

where $\bar{\Delta}_{a b}^{i}\left(z, Q^{2}, 1 / \varepsilon\right)$ is the bare partonic coefficient function before mass factorisation is carried out. Further we have dropped the double index $j j^{\prime}$ because of the vanishing

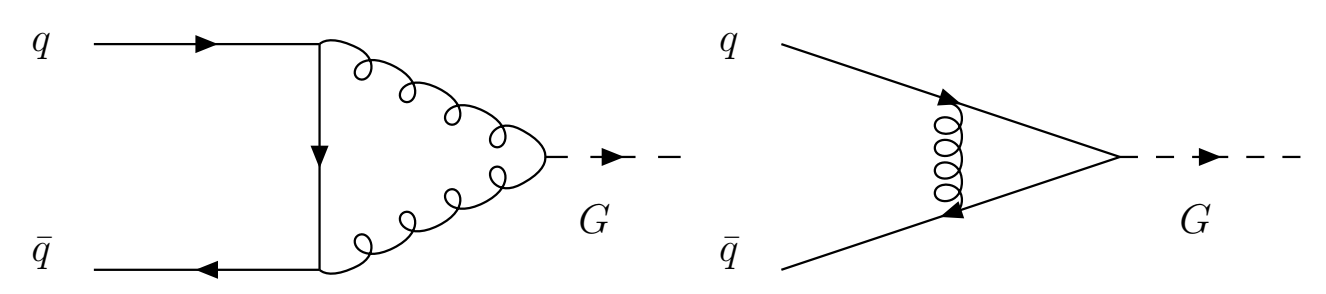

Figure 3: Virtual corrections 

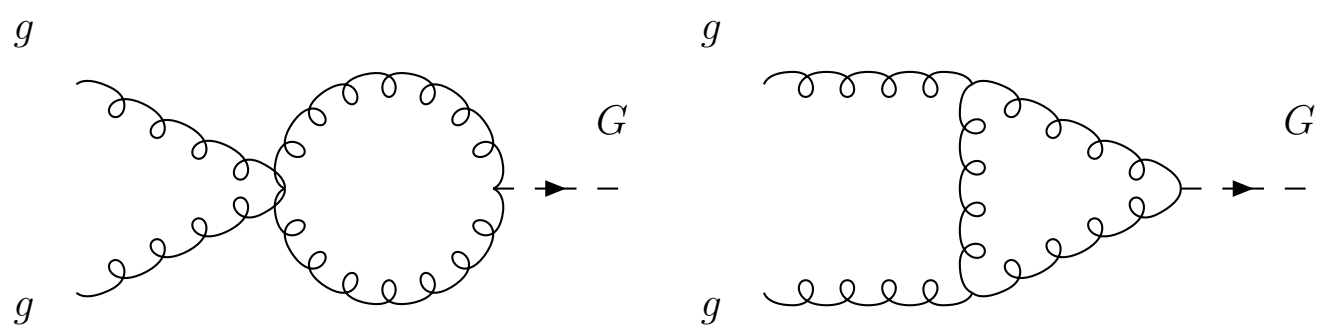

Figure 4: Virtual corrections, gluon loops.

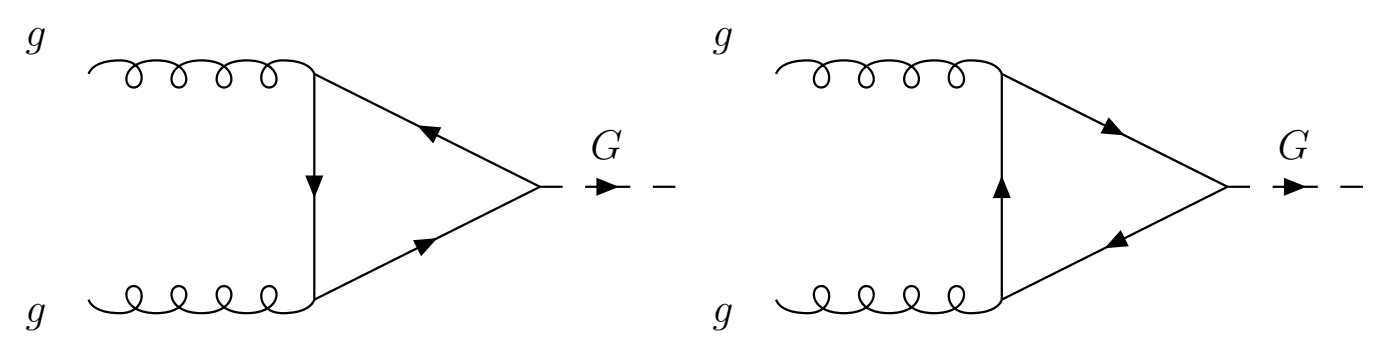

Figure 5: Virtual corrections, quark loops.

interference terms and replace it by the single index $i$ instead. The factorisation scale is given by $\mu_{F}$ and $\otimes$ is the convolution symbol defined as

$$
f(z) \otimes g(z)=\int_{z}^{1} \frac{d y}{y} f(y) g\left(\frac{z}{y}\right),
$$

and the kernel $\Gamma_{c d}(z)$ in the $\overline{M S}$ scheme is given by

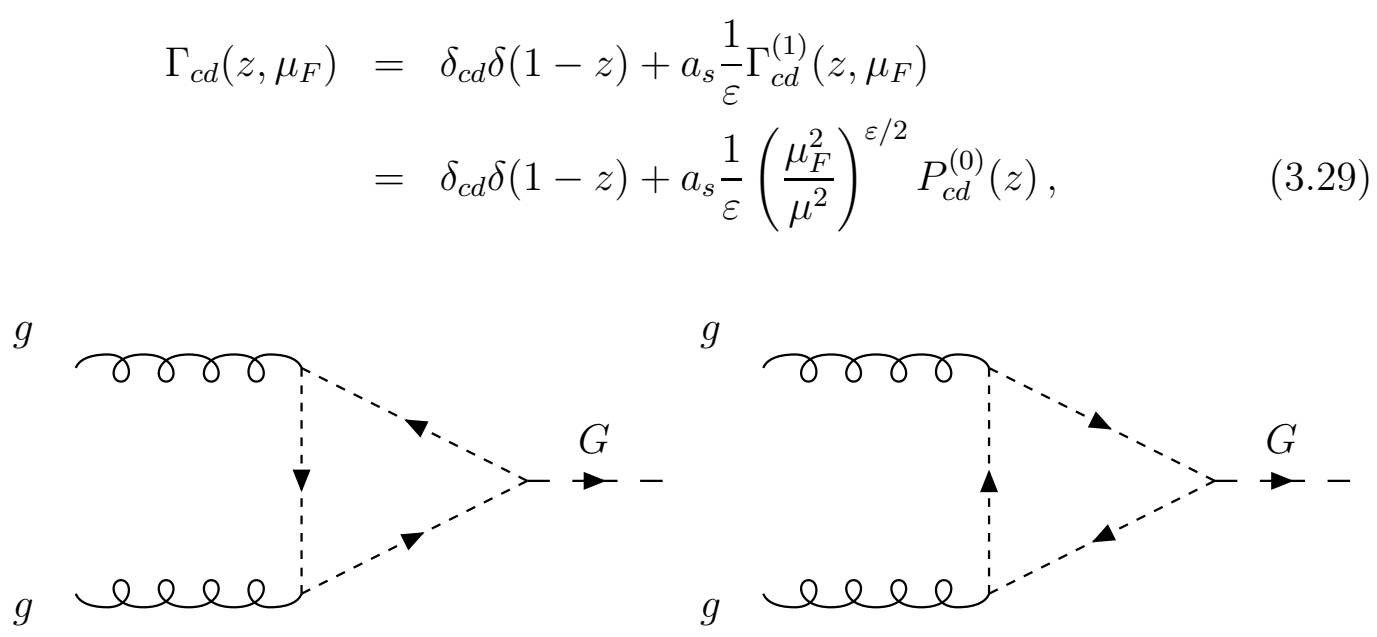

Figure 6: Virtual corrections, ghost loops. 

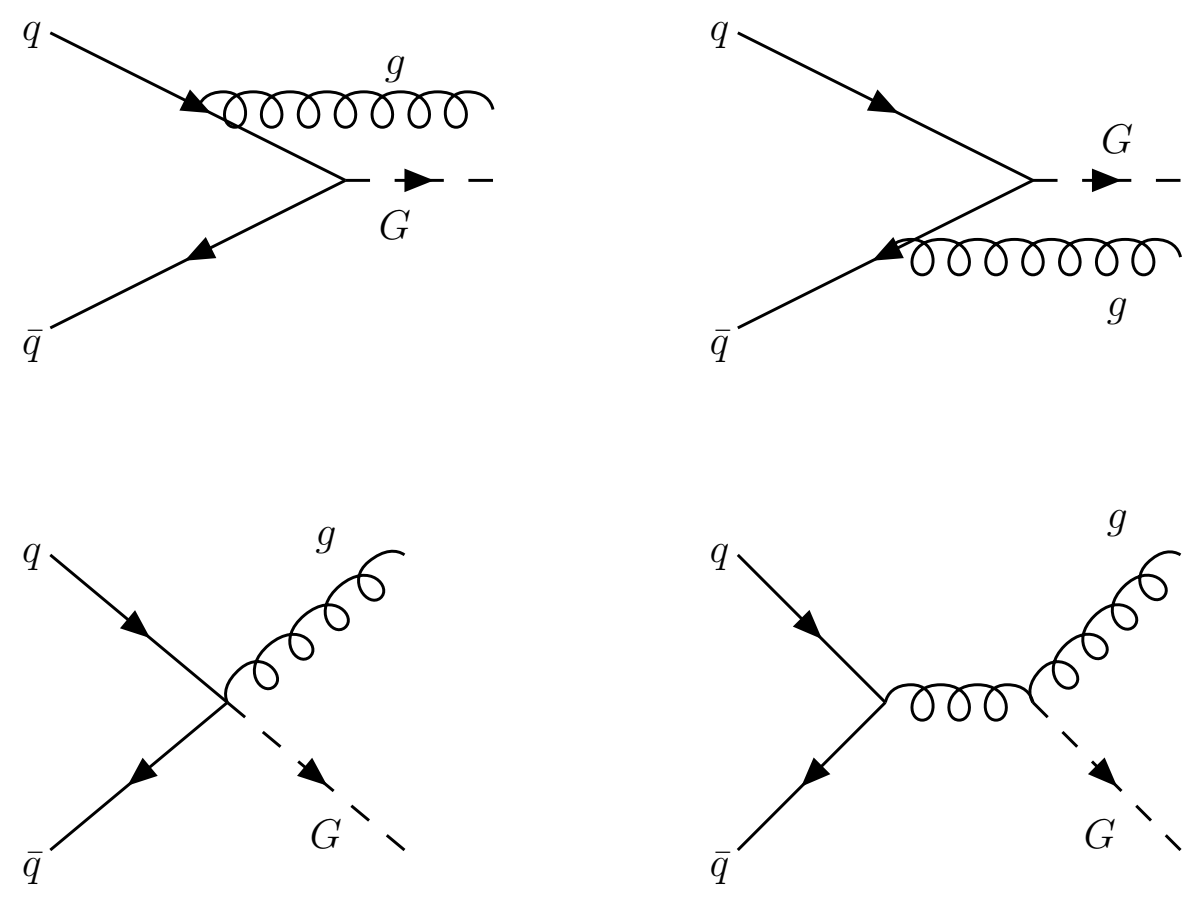

Figure 7: Real emission, $q \bar{q} \rightarrow g G$.

where $P_{c d}^{(0)}(z)$ are the leading order Altarelli-Parisi splitting functions [12],

$$
\Delta_{a b}^{i}=\Delta_{a b}^{(0), i}+a_{s} \Delta_{a b}^{(1), i} .
$$

For convenience we define $a_{s}$ as

$$
a_{s}=\frac{\alpha_{s}\left(\mu_{R}^{2}\right)}{4 \pi} .
$$

Expanding Eq. (3.27) up to order $a_{s}$, we find

$$
\begin{aligned}
\bar{\Delta}_{q \bar{q}}^{\gamma / Z} & =\Delta_{q \bar{q}}^{(0) \gamma / Z}+a_{s} \frac{2}{\varepsilon} \Gamma_{q q}^{(1)} \otimes \Delta_{q \bar{q}}^{(0) \gamma / Z}+a_{s} \Delta_{q \bar{q}}^{(1) \gamma / Z}, \\
\bar{\Delta}_{q g}^{\gamma / Z} & =a_{s} \frac{1}{\varepsilon} \Gamma_{q g}^{(1)} \otimes \Delta_{q \bar{q}}^{(0) \gamma / Z}+a_{s} \Delta_{q g}^{(1) \gamma / Z}, \\
\bar{\Delta}_{q \bar{q}}^{G} & =\Delta_{q \bar{q}}^{(0) G}+a_{s} \frac{2}{\varepsilon} \Gamma_{q q}^{(1)} \otimes \Delta_{q \bar{q}}^{(0) G}+a_{s} \Delta_{q \bar{q}}^{(1) G}, \\
\bar{\Delta}_{q g}^{G} & =a_{s} \frac{1}{\varepsilon}\left(\Gamma_{q g}^{(1)} \otimes \Delta_{q \bar{q}}^{(0) G}+\Gamma_{g q}^{(1)} \otimes \Delta_{g g}^{(0) G}\right)+a_{s} \Delta_{q g}^{(1) G}, \\
\bar{\Delta}_{g g}^{G} & =\Delta_{g g}^{(0) G}+a_{s} \frac{2}{\varepsilon} \Gamma_{g g}^{(1)} \otimes \Delta_{g g}^{(0) G}+a_{s} \Delta_{g g}^{(1) G} .
\end{aligned}
$$



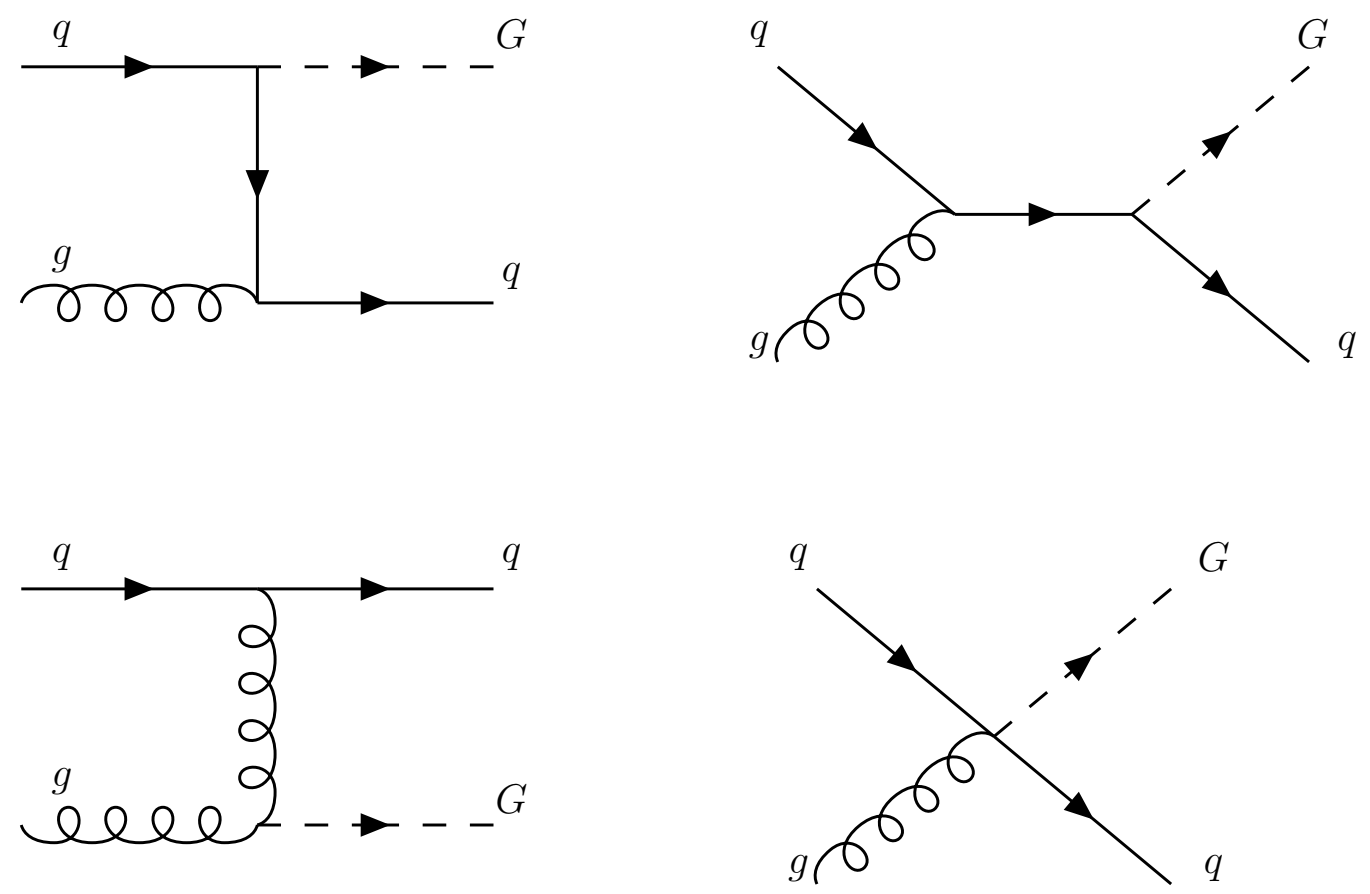

Figure 8: Real emission, $q g \rightarrow q G$.

From the above expressions one can compute the coefficient function $\Delta_{a b}^{i}\left(z, Q^{2}, \mu_{F}\right)$ from the bare $\bar{\Delta}_{a b}^{i}\left(z, Q^{2}, \mu_{F}, 1 / \varepsilon\right)$ and the known Altarelli-Parisi kernels $P_{a b}^{(0)}$. Finally we have to fold these finite $\Delta_{a b}^{i}\left(z, Q^{2}, \mu_{F}\right)$ with the appropriate partonic distribution functions to arrive at the $Q^{2}$ distribution for the DY pair. For completeness we present the results below

$$
\begin{aligned}
2 S \frac{d \sigma^{P_{1} P_{2}}}{d Q^{2}}\left(\tau, Q^{2}\right)= & \sum_{q} \mathcal{F}_{S M, q} \int_{0}^{1} d x_{1} \int_{0}^{1} d x_{2} \int_{0}^{1} d z \delta\left(\tau-z x_{1} x_{2}\right) \\
& \times\left[H_{q \bar{q}}\left(x_{1}, x_{2}, \mu_{F}^{2}\right)\left(\Delta_{q \bar{q}}^{(0), \gamma / Z}\left(z, Q^{2}, \mu_{F}^{2}\right)+a_{s} \Delta_{q \bar{q}}^{(1), \gamma / Z}\left(z, Q^{2}, \mu_{F}^{2}\right)\right)\right. \\
& \left.+\left(H_{q g}\left(x_{1}, x_{2}, \mu_{F}^{2}\right)+H_{g q}\left(x_{1}, x_{2}, \mu_{F}^{2}\right)\right) a_{s} \Delta_{q g}^{(1), \gamma / Z}\left(z, \mu_{F}^{2}\right)\right] \\
& +\sum_{q} \mathcal{F}_{G} \int_{0}^{1} d x_{1} \int_{0}^{1} d x_{2} \int_{0}^{1} d z \delta\left(\tau-z x_{1} x_{2}\right) \\
& \times\left[H_{q \bar{q}}\left(x_{1}, x_{2}, \mu_{F}^{2}\right)\left(\Delta_{q \bar{q}}^{(0), G}\left(z, Q^{2}, \mu_{F}^{2}\right)+a_{s} \Delta_{q \bar{q}}^{(1), G}\left(z, Q^{2}, \mu_{F}^{2}\right)\right)\right.
\end{aligned}
$$



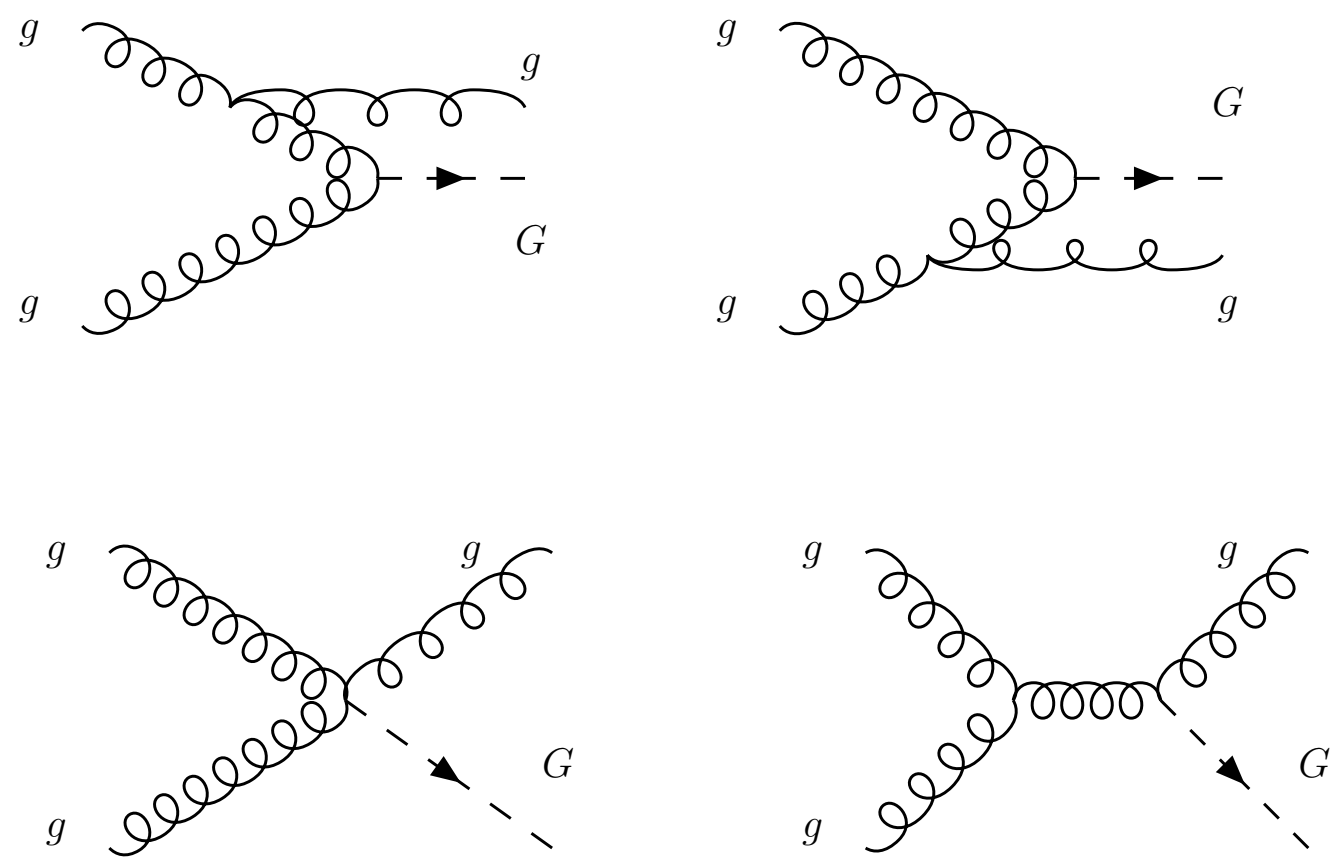

Figure 9: Real emission, $g g \rightarrow g G$.

$$
\begin{aligned}
& +\left(H_{q g}\left(x_{1}, x_{2}, \mu_{F}^{2}\right)+H_{g q}\left(x_{1}, x_{2}, \mu_{F}^{2}\right)\right) a_{s} \Delta_{q g}^{(1), G}\left(z, Q^{2}, \mu_{F}^{2}\right) \\
& \left.+H_{g g}\left(x_{1}, x_{2}, \mu_{F}^{2}\right)\left(\Delta_{g g}^{(0), G}\left(z, Q^{2}, \mu_{F}^{2}\right)+a_{s} \Delta_{g g}^{(1), G}\left(z, Q^{2}, \mu_{F}^{2}\right)\right)\right] .
\end{aligned}
$$

The constants $\mathcal{F}_{S M, q}, \mathcal{F}_{G}$ are given by

$$
\begin{aligned}
\mathcal{F}_{S M, q}= & \frac{4 \alpha^{2}}{3 Q^{2}}\left[Q_{q}^{2}-\frac{2 Q^{2}\left(Q^{2}-M_{Z}^{2}\right)}{\left(\left(Q^{2}-M_{Z}^{2}\right)^{2}+M_{Z}^{2} \Gamma_{Z}^{2}\right) c_{w}^{2} s_{w}^{2}} Q_{q} g_{e}^{V} g_{q}^{V}\right. \\
& \left.+\frac{Q^{4}}{\left(\left(Q^{2}-M_{Z}^{2}\right)^{2}+M_{Z}^{2} \Gamma_{Z}^{2}\right) c_{w}^{4} s_{w}^{4}}\left(\left(g_{e}^{V}\right)^{2}+\left(g_{e}^{A}\right)^{2}\right)\left(\left(g_{q}^{V}\right)^{2}+\left(g_{q}^{A}\right)^{2}\right)\right] \\
\mathcal{F}_{G}= & \frac{\kappa^{4} Q^{6}}{320 \pi^{2}}\left|\mathcal{D}\left(Q^{2}\right)\right|^{2} .
\end{aligned}
$$

The renormalised partonic distributions are

$$
H_{q \bar{q}}\left(x_{1}, x_{2}, \mu_{F}^{2}\right)=f_{q}^{P_{1}}\left(x_{1}, \mu_{F}^{2}\right) f_{\bar{q}}^{P_{2}}\left(x_{2}, \mu_{F}^{2}\right)+f_{\bar{q}}^{P_{1}}\left(x_{1}, \mu_{F}^{2}\right) f_{q}^{P_{2}}\left(x_{2}, \mu_{F}^{2}\right),
$$




$$
\begin{aligned}
& H_{g q}\left(x_{1}, x_{2}, \mu_{F}^{2}\right)=f_{g}^{P_{1}}\left(x_{1}, \mu_{F}^{2}\right)\left(f_{q}^{P_{2}}\left(x_{2}, \mu_{F}^{2}\right)+f_{\bar{q}}^{P_{2}}\left(x_{2}, \mu_{F}^{2}\right)\right), \\
& H_{q g}\left(x_{1}, x_{2}, \mu_{F}^{2}\right)=H_{g q}\left(x_{2}, x_{1}, \mu_{F}^{2}\right), \\
& H_{g g}\left(x_{1}, x_{2}, \mu_{F}^{2}\right)=f_{g}^{P_{1}}\left(x_{1}, \mu_{F}^{2}\right) f_{g}^{P_{2}}\left(x_{2}, \mu_{F}^{2}\right) .
\end{aligned}
$$

The LO coefficient functions are

$$
\begin{aligned}
\Delta_{q \bar{q}}^{(0), \gamma / Z} & =\frac{2 \pi}{N} \delta(1-z), \\
\Delta_{q \bar{q}}^{(0), G} & =\frac{\pi}{8 N} \delta(1-z), \\
\Delta_{g g}^{(0), G} & =\frac{\pi}{2\left(N^{2}-1\right)} \delta(1-z),
\end{aligned}
$$

and the NLO results are given by

$$
\begin{aligned}
\Delta_{q \bar{q}}^{(1) \gamma / Z}= & \left(\frac{2 \pi}{N}\right) 4 C_{F}\left[(-4+2 \zeta(2)) \delta(1-z)+2 \frac{1}{(1-z)_{+}} \ln \left(\frac{Q^{2}}{\mu_{F}^{2}}\right)\right. \\
& +4\left(\frac{\ln (1-z)}{1-z}\right)_{+}+\frac{3}{2} \delta(1-z) \ln \left(\frac{Q^{2}}{\mu_{F}^{2}}\right)-(1+z) \ln \left(\frac{Q^{2}(1-z)^{2}}{\mu_{F}^{2} z}\right) \\
& \left.-2 \frac{\ln (z)}{1-z}\right], \\
\Delta_{q(\bar{q}) g}^{(1) \gamma / Z}= & \left(\frac{2 \pi}{N}\right) T_{F}\left[2\left(1-2 z+2 z^{2}\right) \ln \left(\frac{Q^{2}(1-z)^{2}}{\mu_{F}^{2} z}\right)+1+6 z-7 z^{2}\right], \\
\Delta_{q \bar{q}}^{(1) G}= & \left(\frac{\pi}{8 N}\right) 4 C_{F}\left[(-5+2 \zeta(2)) \delta(1-z)+2 \frac{1}{(1-z)_{+}} \ln \left(\frac{Q^{2}}{\mu_{F}^{2}}\right)\right. \\
& +4\left(\frac{\ln (1-z)}{1-z}\right)_{+}+\frac{3}{2} \delta(1-z) \ln \left(\frac{Q^{2}}{\mu_{F}^{2}}\right)-(1+z) \ln \left(\frac{Q^{2}(1-z)^{2}}{\mu_{F}^{2} z}\right) \\
& \left.-2 \frac{\ln (z)}{1-z}+\frac{4}{3 z}-\frac{4 z^{2}}{3}\right], \\
\Delta_{q(\bar{q}) g}^{(1) G}= & \left(\frac{\pi}{8 N}\right) T_{F}\left[2\left(-7+\frac{8}{z}+2 z+2 z^{2}\right) \ln \left(\frac{Q^{2}(1-z)^{2}}{\mu_{F}^{2} z}\right)\right.
\end{aligned}
$$




$$
\begin{aligned}
& \left.+9-\frac{12}{z}+18 z-7 z^{2}\right] \\
\Delta_{g g}^{(1) G}= & \left(\frac{\pi}{2\left(N^{2}-1\right)}\right) C_{A}\left[\left(-\frac{203}{9}+8 \zeta(2)\right) \delta(1-z)+8 \frac{1}{(1-z)_{+}} \ln \left(\frac{Q^{2}}{\mu_{F}^{2}}\right)\right. \\
& +16\left(\frac{\ln (1-z)}{1-z}\right)_{+}+\frac{22}{3} \delta(1-z) \ln \left(\frac{Q^{2}}{\mu_{F}^{2}}\right)+8\left(-2+\frac{1}{z}+z-z^{2}\right) \\
& \left.\times \ln \left(\frac{Q^{2}(1-z)^{2}}{\mu_{F}^{2} z}\right)-8 \frac{\ln (z)}{(1-z)}-2-\frac{22}{3 z}+2 z+\frac{22 z^{2}}{3}\right] \\
& +\left(\frac{\pi}{2\left(N^{2}-1\right)}\right) n_{f} T_{F}\left[\left(\frac{70}{9}-\frac{8}{3} \ln \left(\frac{Q^{2}}{\mu_{F}^{2}}\right)\right) \delta(1-z)\right]
\end{aligned}
$$

For $S U(N)$ the colour factors in the above equations are

$$
C_{F}=\frac{N^{2}-1}{2 N}, \quad C_{A}=N, \quad T_{F}=1 / 2,
$$

and $n_{f}$ is the number of flavours. The "plus" functions appearing in the above results are the distributions which satisfy the following equation

$$
\begin{aligned}
f_{+}(z) & =\left(\frac{\ln ^{i}(1-z)}{1-z}\right)_{+}, \quad f(z)=\left(\frac{\ln ^{i}(1-z)}{1-z}\right), \\
\int_{0}^{1} d z f_{+}(z) g(z) & =\int_{0}^{1} d z f(z)(g(z)-g(1)),
\end{aligned}
$$

where $g(z)$ is any well behaved function in the region $0 \leq z \leq 1$. 


\section{Differential cross section with respect to $x_{F}$}

In this section we compute the $x_{F}$-distribution of the di-muon pair up to NLO in QCD

with the $\gamma, Z$ and the graviton in the intermediate state. The NLO corrections to processes containing an intermediate photon were already calculated in [6], [13]-[16]. The variable $x_{F}$ is defined as

$$
x_{F}=\frac{2\left(p_{1}-p_{2}\right) \cdot q}{S}
$$

where $p_{1}, p_{2}$ are the momenta of incoming hadrons and $q$ is the sum of final state muon momenta, $q=l_{1}+l_{2}$. In the CM frame of the hadrons $(\mathrm{CMH})$

$$
x_{F}=\frac{2 q_{C M H}^{3}}{\sqrt{S}},
$$

where $q_{C M H}^{3}$ is the third component of $q$ in the CM frame of Hadrons. The hadronic $x_{F}$ distribution can be obtained by introducing the identity

$$
\int d x_{F} \delta\left(x_{F}-\frac{2\left(p_{1}-p_{2}\right) \cdot q}{S}\right)=1
$$

in Eq.(3.2) and bringing the measure $d x_{F}$ to the left. Hence we find

$$
2 S \frac{d \sigma^{P_{1} P_{2}}}{d Q^{2} d x_{F}}\left(\tau, x_{F}, Q^{2}\right)=\frac{1}{2 \pi} \sum_{j j^{\prime}=\gamma, Z, G} \tilde{P}_{j}\left(Q^{2}\right) \tilde{P}_{j^{\prime}}^{*}\left(Q^{2}\right) L_{j j^{\prime}}\left(Q^{2}\right) \frac{d W_{j j^{\prime}}^{P_{1} P_{2}}}{d x_{F}}\left(\tau, x_{F}, Q^{2}\right) .
$$

The hadronic structure function is defined as

$$
\begin{aligned}
\frac{d W_{j j^{\prime}}^{P_{1} P_{2}}}{d x_{F}}\left(\tau, x_{F}, Q^{2}\right)= & \sum_{a b, j j^{\prime}} \int d x_{1} \int d x_{2} f_{a}^{P_{1}}\left(x_{1}\right) f_{b}^{P_{2}}\left(x_{2}\right) \\
& \times \int d z \int d P S_{m+1}\left|M^{a b \rightarrow j j^{\prime}}\right|^{2} T_{j j^{\prime}}(q) \delta\left(\tau-z x_{1} x_{2}\right) \\
& \times \delta\left(x_{F}-\frac{2\left(p_{1}-p_{2}\right) \cdot q}{S}\right) .
\end{aligned}
$$

We start by computing the Born contribution. This involves the computation of the matrix element squared for process $a\left(k_{1}\right)+b\left(k_{2}\right) \rightarrow j j^{\prime}(-q)$ and the $(0+1)$-body phase space integration as well as the $d z$ integration. The $(0+1)$-body phase space is computed using Eq. (3.5) and one finds

$$
\int d P S_{0+1}=\frac{2 \pi}{Q^{2}} \delta(z-1) .
$$


To compute the Born process $a\left(k_{1}\right)+b\left(k_{2}\right) \rightarrow j j^{\prime}(-q)$, we choose the following parameterisation for the momenta

$$
k_{1}=\frac{\sqrt{\hat{s}}}{2}(1,0, \cdots, 0,1), \quad k_{2}=\frac{\sqrt{\hat{s}}}{2}(1,0, \cdots, 0,-1), \quad-q=\sqrt{\hat{s}}(1,0, \cdots, 0,0) .
$$

Subsequently we express the partonic momenta in terms of the hadronic momenta using the scaling variables $x_{1}, x_{2}$ as $k_{1}=x_{1} p_{1}, k_{2}=x_{2} p_{2}$

$$
\begin{aligned}
\int d P S_{0+1} \int d z \delta\left(x_{F}-\frac{2\left(p_{1}-p_{2}\right) \cdot q}{S}\right) \delta\left(\tau-z x_{1} x_{2}\right)= & \frac{2 \pi}{Q^{2}} \int d z \delta\left(x_{F}-x_{1}+x_{2}\right) \\
& \times \delta(z-1) \delta\left(\tau-z x_{1} x_{2}\right) .
\end{aligned}
$$

We choose to work with the variables $x_{1}^{0}, x_{2}^{0}$ which are defined through

$$
x_{F} \equiv x_{1}^{0}-x_{2}^{0}, \quad \tau \equiv x_{1}^{0} x_{2}^{0} .
$$

Solving the above equations, we find

$$
x_{1}^{0}=\frac{1}{2}\left(x_{F}+\sqrt{x_{F}^{2}+4 \tau}\right), \quad x_{2}^{0}=\frac{1}{2}\left(-x_{F}+\sqrt{x_{F}^{2}+4 \tau}\right) .
$$

After rewriting the arguments of the delta functions in terms of the variables $x_{1}^{0}, x_{2}^{0}$, and performing the $z$ integration using $\delta\left(\tau-z x_{1} x_{2}\right)$, we arrive at a simple looking expression for Eq. (4.8) as

$$
\begin{gathered}
\frac{2 \pi}{Q^{2}} \int d z \delta(z-1) \delta\left(x_{F}-x_{1}+x_{2}\right) \delta\left(\tau-z x_{1} x_{2}\right)\left|M^{a b \rightarrow j j^{\prime}}\right|^{2} \cdot T_{j j^{\prime}}= \\
\left.\frac{2 \pi}{Q^{2}} \frac{\delta\left(x_{1}-x_{1}^{0}\right) \delta\left(x_{2}-x_{2}^{0}\right)}{x_{1}^{0}+x_{2}^{0}}\left|M^{a b \rightarrow j j^{\prime}}\right|^{2} \cdot T_{j j^{\prime}}\right|_{z=1} .
\end{gathered}
$$

Finally, the Born matrix element squared

$$
\left|M^{a b \rightarrow j j^{\prime}}\right|^{2} \cdot T_{j j^{\prime}}
$$

is computed using the parameterisations given in Eq. (4.7). It is then substituted in Eq. (4.5) to arrive at the leading order $x_{F}$ distribution. Our next task is to compute the next to leading order contributions to the Born $x_{F}$ distribution. This involves the computation of the matrix element squared for the processes $a\left(k_{1}\right)+b\left(k_{2}\right) \rightarrow$ $j j^{\prime}(-q)+c(-k)$ and $1+1$ phase space integration. Since the integral on the right hand 
side of Eq. (4.4) is Lorentz invariant, we can work in the CM frame of the incoming partons. In this frame the momenta of the particles are parametrised as

$$
\begin{aligned}
k_{1} & =\frac{\sqrt{\hat{s}}}{2}(1,0, \cdots, 0,1), \quad k_{2}=\frac{\sqrt{\hat{s}}}{2}(1,0, \cdots, 0,-1), \\
-q & =\frac{\sqrt{\hat{s}}}{2}(1+z, 0, \cdots,-(1-z) \sin \theta,-(1-z) \cos \theta), \\
-k & =\frac{\sqrt{\hat{s}}}{2}(1-z, 0, \cdots,(1-z) \sin \theta,(1-z) \cos \theta) .
\end{aligned}
$$

In this frame, the $1+1$ phase space becomes

$$
\int d P S_{1+1}=\frac{1}{8 \pi}\left(\frac{Q^{2}}{4 \pi}\right)^{\varepsilon / 2} \frac{1}{\Gamma(1+\varepsilon / 2)} z^{-\varepsilon / 2}(1-z)^{1+\varepsilon} \int_{0}^{1} d y(y(1-y))^{\varepsilon / 2}
$$

where $y$ is related to $\cos \theta$ by $\cos \theta=2 y-1$. The $x_{F}$ delta function becomes

$$
\delta\left(x_{F}-\frac{2\left(p_{1}-p_{2}\right) \cdot q}{S}\right)=\delta\left(x_{F}-x_{1}+z x_{2}+y(1-z)\left(x_{1}+x_{2}\right)\right) .
$$

We can perform $y$ as well as $z$ integrations by writing the delta functions in terms of the variables $x_{1}^{0}, x_{2}^{0}$ as

$$
\begin{aligned}
\delta\left(x_{F}-x_{1}+z x_{2}+y(1-z)\left(x_{1}+x_{2}\right)\right) & =\frac{x_{1} x_{2}}{\left(x_{1}+x_{2}\right)\left(x_{1} x_{2}-x_{1}^{0} x_{2}^{0}\right)} \delta\left(y-y^{*}\right) \\
\delta\left(\tau-z x_{1} x_{2}\right) & =\frac{1}{x_{1} x_{2}} \delta\left(z-z^{*}\right)
\end{aligned}
$$

where

$$
y^{*}=\frac{x_{2}\left(x_{1}-x_{1}^{0}\right)\left(x_{1}+x_{2}^{0}\right)}{\left(x_{1} x_{2}-x_{1}^{0} x_{2}^{0}\right)\left(x_{1}+x_{2}\right)}, \quad z^{*}=\frac{x_{1}^{0} x_{2}^{0}}{x_{1} x_{2}} .
$$

In other words

$$
\begin{aligned}
& \int d P S_{1+1} \int d z \delta\left(\tau-z x_{1} x_{2}\right) \delta\left(x_{F}-\frac{2\left(p_{1}-p_{2}\right) \cdot q}{S}\right)\left|M^{a b \rightarrow j j^{\prime}}\right|^{2} \cdot T_{j j^{\prime}}= \\
& \frac{1}{8 \pi}\left(\frac{Q^{2}}{4 \pi}\right)^{\varepsilon / 2} \frac{1}{\Gamma(1+\varepsilon / 2)} \frac{\left(x_{1}^{0} x_{2}^{0}\right)^{-\frac{\varepsilon}{2}}\left(x_{1}+x_{2}\right)^{-\varepsilon}}{x_{1} x_{2}\left(x_{1}+x_{2}\right)} \\
& \times\left.\left(\left(x_{1}-x_{1}^{0}\right)\left(x_{1}+x_{2}^{0}\right)\left(x_{2}-x_{2}^{0}\right)\left(x_{2}+x_{1}^{0}\right)\right)^{\varepsilon / 2}\left|M^{a b \rightarrow j j^{\prime}}\right|^{2} \cdot T_{j j^{\prime}}\right|_{y=y^{*}, z=z^{*}}
\end{aligned}
$$


Notice that the matrix elements $\left|M^{a b \rightarrow j j^{\prime}}\right|^{2} \cdot T_{j j^{\prime}}$ are evaluated for $y$ and $z$ given by the delta function constraints given in Eq. (4.16) and Eq. (4.17). To this order, one has to include the virtual corrections to the Born processes as well. We use the same formula given in Eq. (4.6) with the one loop corrected matrix elements. The sum of real emission contributions and the one loop corrections to the Born processes is free of the soft singularities as expected. Therefore the result contains only the collinear divergences which can be removed by the standard mass factorisation. This is done by redefining the parton densities using the Altarelli-Parisi kernels as follows

$$
f_{a}^{P}(z)=\sum_{b} \Gamma_{a b}^{-1} \otimes f_{b}^{P}\left(z, \mu_{F}^{2}\right),
$$

which implies

$$
\begin{aligned}
f_{q}^{P}(z)= & f_{q}^{P}\left(z, \mu_{F}^{2}\right)-a_{s} \frac{1}{\varepsilon} \Gamma_{q q}^{(1)} \otimes f_{q}^{P}\left(z, \mu_{F}^{2}\right)-a_{s} \frac{1}{\varepsilon} \Gamma_{q g}^{(1)} \otimes f_{g}^{P}\left(z, \mu_{F}^{2}\right), \\
f_{\bar{q}}^{P}(z)= & f_{\bar{q}}^{P}\left(z, \mu_{F}^{2}\right)-a_{s} \frac{1}{\varepsilon} \Gamma_{\bar{q} \bar{q}}^{(1)} \otimes f_{\bar{q}}^{P}\left(z, \mu_{F}^{2}\right)-a_{s} \frac{1}{\varepsilon} \Gamma_{\bar{q} g}^{(1)} \otimes f_{g}^{P}\left(z, \mu_{F}^{2}\right), \\
f_{g}^{P}(z)= & f_{g}^{P}\left(z, \mu_{F}^{2}\right)-a_{s} \frac{1}{\varepsilon} n_{f}\left(\Gamma_{g q}^{(1)} \otimes f_{q}^{P}\left(z, \mu_{F}^{2}\right)+\Gamma_{g \bar{q}}^{(1)} \otimes f_{\bar{q}}^{P}\left(z, \mu_{F}^{2}\right)\right) \\
& -a_{s} \frac{1}{\varepsilon} \Gamma_{g g}^{(1)} \otimes f_{g}^{P}\left(z, \mu_{F}^{2}\right) .
\end{aligned}
$$

Finally we arrive at

$$
\begin{aligned}
& 2 S \frac{d \sigma^{P_{1} P_{2}}}{d Q^{2} d x_{F}}\left(\tau, x_{F}, Q^{2}\right)= \\
& \quad \sum_{i=q} \mathcal{F}_{S M, q}\left(C_{q \bar{q}}^{S M}\left(x_{1}^{0}, x_{2}^{0}, \mu_{F}^{2}\right)+C_{q g}^{S M}\left(x_{1}^{0}, x_{2}^{0}, \mu_{F}^{2}\right)+C_{g q}^{S M}\left(x_{1}^{0}, x_{2}^{0}, \mu_{F}^{2}\right)\right) \\
& \quad+\sum_{i=q} \mathcal{F}_{G}\left(C_{q \bar{q}}^{G}\left(x_{1}^{0}, x_{2}^{0}, \mu_{F}^{2}\right)+C_{q g}^{G}\left(x_{1}^{0}, x_{2}^{0}, \mu_{F}^{2}\right)+C_{g q}^{G}\left(x_{1}^{0}, x_{2}^{0}, \mu_{F}^{2}\right)\right) \\
& \quad+\mathcal{F}_{G} C_{g g}^{G}\left(x_{1}^{0}, x_{2}^{0}, \mu_{F}^{2}\right),
\end{aligned}
$$

where

$$
\begin{aligned}
C_{a b}^{S M}\left(x_{1}^{0}, x_{2}^{0}, \mu_{F}^{2}\right) & =C_{a b}^{S M,(0)}\left(x_{1}^{0}, x_{2}^{0}, \mu_{F}^{2}\right)+a_{s} C_{a b}^{S M,(1)}\left(x_{1}^{0}, x_{2}^{0}, \mu_{F}^{2}\right), \\
C_{a b}^{G}\left(x_{1}^{0}, x_{2}^{0}, \mu_{F}^{2}\right) & =C_{a b}^{G,(0)}\left(x_{1}^{0}, x_{2}^{0}, \mu_{F}^{2}\right)+a_{s} C_{a b}^{G,(1)}\left(x_{1}^{0}, x_{2}^{0}, \mu_{F}^{2}\right) .
\end{aligned}
$$

We have presented $C_{a b}^{S M,(0)}, C_{a b}^{S M,(1)}, C_{a b}^{G,(0)}$ and $C_{a b}^{G,(1)}$ in appendix A. 


\section{Differential cross section with respect to $Y$}

In this section we compute the rapidity distribution of di-lepton pair up to NLO in QCD with $\gamma, Z$ and graviton in the intermediate state. The NLO corrections to processes containing an intermediate photon were already calculated in [6], [13]-[16]. We define $Y$ as

$$
Y=\frac{1}{2} \log \left(\frac{p_{2} \cdot q}{p_{1} \cdot q}\right)
$$

In the CM frame of the hadron $(C M H)$, this is nothing but

$$
Y=\frac{1}{2} \log \left(\frac{q_{C M H}^{0}-q_{C M H}^{3}}{q_{C M H}^{0}+q_{C M H}^{3}}\right) .
$$

With this definition the distribution in $Y$ can be computed by introducing the identity

$$
\int d Y \delta\left(Y-\frac{1}{2} \log \left(\frac{p_{2} \cdot q}{p_{1} \cdot q}\right)\right)=1
$$

in Eq. (3.2) and bringing the measure $d Y$ to the left. Hence we find

$$
2 S \frac{d \sigma^{P_{1} P_{2}}}{d Q^{2} d Y}\left(\tau, Y, Q^{2}\right)=\frac{1}{2 \pi} \sum_{j j^{\prime}=\gamma, Z, G} \tilde{P}_{j}\left(Q^{2}\right) \tilde{P}_{j^{\prime}}^{*}\left(Q^{2}\right) L_{j j^{\prime}}\left(Q^{2}\right) \frac{d W_{j j^{\prime}}^{P_{1} P_{2}}}{d Y}\left(\tau, Y, Q^{2}\right) .
$$

The hadronic structure function equals

$$
\begin{aligned}
\frac{d W_{j j^{\prime}}^{P_{1} P_{2}}}{d Y}\left(\tau, Y, Q^{2}\right)= & \sum_{a b, j j^{\prime}} \int d x_{1} \int d x_{2} f_{a}^{P_{1}}\left(x_{1}\right) f_{b}^{P_{2}}\left(x_{2}\right) \\
& \times \int d z \int d P S_{m+1}\left|M^{a b \rightarrow j j^{\prime}}\right|^{2} T_{j j^{\prime}}(q) \delta\left(\tau-z x_{1} x_{2}\right) \\
& \times \delta\left(Y-\frac{1}{2} \log \left(\frac{p_{2} \cdot q}{p_{1} \cdot q}\right)\right) .
\end{aligned}
$$

We first compute the matrix element squared for $a+b \rightarrow j j^{\prime}$ and then substitute this in Eq. (5.4). Then we perform the $(0+1)$-body phase space integration as well as $z$ integration. We follow the same steps as for the $x_{F}$ distribution. Using Eq. (4.6) for the $(0+1)$-body phase space and performing $z$ integration, we obtain

$$
\begin{aligned}
& \int d P S_{0+1} \int d z \delta\left(Y-\frac{1}{2} \log \left(\frac{p_{2} \cdot q}{p_{1} \cdot q}\right)\right) \delta\left(\tau-z x_{1} x_{2}\right)=\frac{2 \pi}{Q^{2}} \int d z \delta\left(Y-\frac{1}{2} \log \left(\frac{x_{1}}{x_{2}}\right)\right) \\
& \times \delta(z-1) \delta\left(\tau-z x_{1} x_{2}\right) .
\end{aligned}
$$


Instead of working with the variables $Y, \tau$, we choose to work with $x_{1}^{0}, x_{2}^{0}$ which are defined as

$$
Y=\frac{1}{2} \log \left(\frac{x_{1}^{0}}{x_{2}^{0}}\right), \quad \tau=x_{1}^{0} x_{2}^{0} .
$$

Solving the above equations, we obtain

$$
x_{1}^{0}=\sqrt{\tau} e^{Y}, \quad x_{2}^{0}=\sqrt{\tau} e^{-Y} .
$$

We can perform the $z$ integration in Eq. (5.6) using $\delta\left(\tau-z x_{1} x_{2}\right)$ after rewriting the remaining delta functions in terms of the variables $x_{1}^{0}, x_{2}^{0}$. We then get

$$
\begin{gathered}
\frac{2 \pi}{Q^{2}} \int d z \delta(z-1) \delta\left(Y-\frac{1}{2} \log \left(\frac{x_{1}}{x_{2}}\right)\right) \delta\left(\tau-z x_{1} x_{2}\right)\left|M^{a b \rightarrow j j^{\prime}}\right|^{2} T_{j j^{\prime}}= \\
\left.\frac{2 \pi}{Q^{2}} \delta\left(x_{1}-x_{1}^{0}\right) \delta\left(x_{2}-x_{2}^{0}\right)\left|M^{a b \rightarrow j j^{\prime}}\right|^{2} T_{j j^{\prime}}\right|_{z=1} \cdot
\end{gathered}
$$

Next we evaluate the NLO correction to the Born processes. This involves the computation of $1+1$ phase space for the process $a\left(k_{1}\right)+b\left(k_{2}\right) \rightarrow j j^{\prime}(-q)+c(-k)$. Since the integral on the right hand side of Eq. (5.5) is Lorentz invariant, we can work in the $\mathrm{CM}$ frame of the incoming partons. We follow again the derivation of $x_{F}$ distribution. The $Y$ delta function in this frame becomes

$$
\delta\left(Y-\frac{1}{2} \log \left(\frac{p_{2} \cdot q}{p_{1} \cdot q}\right)\right)=\delta\left(Y-\frac{1}{2} \log \left(\frac{x_{1}(1-y(1-z))}{x_{2}(z+y(1-z))}\right)\right) .
$$

The two delta functions reduce to

$$
\begin{aligned}
\delta\left(Y-\frac{1}{2} \log \left(\frac{x_{1}(1-y(1-z))}{x_{2}(z+y(1-z))}\right)\right) & =\frac{2 x_{1} x_{2} x_{1}^{0} x_{2}^{0}\left(x_{1} x_{2}+x_{1}^{0} x_{2}^{0}\right)}{\left(x_{1} x_{2}-x_{1}^{0} x_{2}^{0}\right)\left(x_{1} x_{2}^{0}+x_{1}^{0} x_{2}\right)^{2}} \delta\left(y-y^{*}\right) \\
\delta\left(\tau-z x_{1} x_{2}\right) & =\frac{1}{x_{1} x_{2}} \delta\left(z-z^{*}\right)
\end{aligned}
$$

where

$$
y^{*}=\frac{x_{2} x_{2}^{0}\left(x_{1}+x_{1}^{0}\right)\left(x_{1}-x_{1}^{0}\right)}{\left(x_{1} x_{2}^{0}+x_{2} x_{1}^{0}\right)\left(x_{1} x_{2}-x_{1}^{0} x_{2}^{0}\right)}, \quad z^{*}=\frac{x_{1}^{0} x_{2}^{0}}{x_{1} x_{2}} .
$$

Using Eqs. (5.11) and (5.12) we arrive at

$$
\begin{gathered}
\int d P S_{1+1} \int d z \delta\left(\tau-z x_{1} x_{2}\right) \delta\left(Y-\frac{1}{2} \log \left(\frac{p_{2} \cdot q}{p_{1} \cdot q}\right)\right)\left|M^{a b \rightarrow j j^{\prime}}\right|^{2} T_{j j^{\prime}}= \\
\frac{1}{8 \pi}\left(\frac{Q^{2}}{4 \pi}\right)^{\varepsilon / 2} \frac{1}{\Gamma(1+\varepsilon / 2)} \frac{2 x_{1}^{0} x_{2}^{0}\left(x_{1} x_{2}+x_{1}^{0} x_{2}^{0}\right)}{x_{1} x_{2}\left(x_{1} x_{2}^{0}+x_{2} x_{1}^{0}\right)^{2}}\left(x_{1} x_{2}^{0}+x_{1}^{0} x_{2}\right)^{-\varepsilon} \\
\left.\left(\left(x_{1}-x_{1}^{0}\right)\left(x_{1}+x_{1}^{0}\right)\left(x_{2}-x_{2}^{0}\right)\left(x_{2}+x_{2}^{0}\right)\right)^{\varepsilon / 2}\left|M^{a b \rightarrow j j^{\prime}}\right|^{2} T_{j j^{\prime}}\right|_{y=y^{*}, z=z^{*}}
\end{gathered}
$$


We substitute Eq. (5.13) in Eq. (5.5) to obtain the real emission contributions to the $Y$ distribution. The virtual corrections to the Born processes can be obtained using Eq. (4.6) with the one loop corrected matrix elements. After adding the real emission contributions and virtual corrections to the Born processes, all soft singularities cancel. The remaining collinear divergences are removed by mass factorisation using Eq. (4.20). Finally we arrive at the finite results for the $Y$ distribution

$$
\begin{aligned}
2 S \frac{d \sigma^{P_{1} P_{2}}}{d Q^{2} d Y}\left(\tau, Y, Q^{2}\right)= & \sum_{i=q} \mathcal{F}_{S M, q}\left(D_{q \bar{q}}^{S M}\left(x_{1}^{0}, x_{2}^{0}, \mu_{F}^{2}\right)+D_{q g}^{S M}\left(x_{1}^{0}, x_{2}^{0}, \mu_{F}^{2}\right)\right. \\
& \left.+D_{g q}^{S M}\left(x_{1}^{0}, x_{2}^{0}, \mu_{F}^{2}\right)\right)+\sum_{i=q} \mathcal{F}_{G}\left(D_{q \bar{q}}^{G}\left(x_{1}^{0}, x_{2}^{0}, \mu_{F}^{2}\right)\right. \\
& \left.+D_{q g}^{G}\left(x_{1}^{0}, x_{2}^{0}, \mu_{F}^{2}\right)+D_{g q}^{G}\left(x_{1}^{0}, x_{2}^{0}, \mu_{F}^{2}\right)\right) \\
& +\mathcal{F}_{G} D_{g g}^{G}\left(x_{1}^{0}, x_{2}^{0}, \mu_{F}^{2}\right)
\end{aligned}
$$

where

$$
\begin{aligned}
D_{a b}^{S M}\left(x_{1}^{0}, x_{2}^{0}, \mu_{F}^{2}\right) & =D_{a b}^{S M,(0)}\left(x_{1}^{0}, x_{2}^{0}, \mu_{F}^{2}\right)+a_{s} D_{a b}^{S M,(1)}\left(x_{1}^{0}, x_{2}^{0}, \mu_{F}^{2}\right) \\
D_{a b}^{G}\left(x_{1}^{0}, x_{2}^{0}, \mu_{F}^{2}\right) & =D_{a b}^{G,(0)}\left(x_{1}^{0}, x_{2}^{0}, \mu_{F}^{2}\right)+a_{s} D_{a b}^{G,(1)}\left(x_{1}^{0}, x_{2}^{0}, \mu_{F}^{2}\right)
\end{aligned}
$$

We have presented $D_{a b}^{S M,(0)}, D_{a b}^{S M,(1)} D_{a b}^{G,(0)}$ and $D_{a b}^{G,(1)}$ in appendix B. 


\section{Forward Backward asymmetry $A_{F B}$}

In this section, we compute the forward backward asymmetry of the di-lepton pair. The NLO correction to $A_{F B}$ in the SM were calculated in [19]. The forward backward asymmetry is computed using the distribution define by

$$
2 S \frac{d \delta \sigma^{P_{1} P_{2}}}{d Q^{2}}\left(\tau, Q^{2}\right)=\left(\int_{0}^{1}-\int_{-1}^{0}\right) d \cos \theta^{*} 2 S \frac{d \sigma^{P_{1} P_{2}}}{d Q^{2} d \cos \theta^{*}}\left(\tau, Q^{2}, \cos \theta^{*}\right)
$$

where $\cos \theta^{*}$ is the angle between the final state lepton (say $l_{1}$ ) momentum and the

initial state hadron (say $p_{1}$ ) momentum in the CM frame of the lepton pair. In general it can be written as

$$
\cos \theta^{*}=\frac{p_{1} \cdot\left(l_{1}-l_{2}\right)}{p_{1} \cdot\left(l_{1}+l_{2}\right)}
$$

The computation of the above distribution is quite similar to that of $d \sigma / d Q^{2}$. In the QCD improved parton model, we find

$$
\begin{aligned}
2 S \frac{d \sigma^{P_{1} P_{2}}}{d Q^{2} d \cos \theta^{*}}\left(\tau, Q^{2}\right)= & \sum_{a b=q, \bar{q}, g} \int_{0}^{1} d x_{1} \int_{0}^{1} d x_{2} f_{a}^{P_{1}}\left(x_{1}\right) f_{b}^{P_{2}}\left(x_{2}\right) \\
& \times \int_{0}^{1} d z 2 \hat{s} \frac{d \hat{\sigma}^{a b}}{d Q^{2} d \cos \theta^{*}}\left(z, Q^{2}\right) \delta\left(\tau-z x_{1} x_{2}\right) .
\end{aligned}
$$

We compute the partonic cross section using

$$
\begin{aligned}
2 \hat{s} \frac{d \hat{\sigma}^{a b}}{d Q^{2} d \cos \theta^{*}}= & \frac{1}{2 \pi} \sum_{j j^{\prime}=\gamma, Z, G} \int d P S_{m+1}\left|M^{a b \rightarrow j j^{\prime}}\right|^{2} \cdot P_{j}(q) \cdot P_{j^{\prime}}^{*}(q) \cdot \\
& \mathcal{L}^{j j^{\prime} \rightarrow l^{+} l^{-}}\left(q, \cos \theta^{*}\right)
\end{aligned}
$$

where $\mathcal{L}^{j j^{\prime} \rightarrow l^{+} l^{-}}\left(q, \cos \theta^{*}\right)$ can be computed using

$$
\begin{aligned}
\mathcal{L}^{j j^{\prime} \rightarrow \mu^{+} \mu^{-}}\left(q, \cos \theta^{*}\right)= & \int \prod_{i=1}^{2}\left(\frac{d^{n} l_{i}}{(2 \pi)^{n}} 2 \pi \delta^{+}\left(l_{i}^{2}\right)\right)(2 \pi)^{n} \delta^{(n)}\left(q-l_{1}-l_{2}\right) \\
& \times \delta\left(\cos \theta^{*}-\frac{p_{1} \cdot\left(l_{1}-l_{2}\right)}{p_{1} \cdot\left(l_{1}+l_{2}\right)}\right)\left|M^{j j^{\prime} \rightarrow \mu^{+} \mu^{-}}\right|^{2}
\end{aligned}
$$

The computation of the leptonic part using the above formula is straight forward. The hadronic part again involves the computation of various processes that contribute to $Q$ distribution. Since we are looking at the angular distributions which are "odd" in 
$\cos \theta^{*}$, the contributions come mainly from interferences. The non-vanishing contribution in the standard model sector comes from the interference of photon mediated processes with $Z$-boson mediated processes. We also find that non-vanishing contributions come from the interference of standard model diagrams with the graviton exchange diagrams. These inference diagrams are absent in the computation of $Q, X_{F}$ and rapidity distributions because they are odd in $\cos \theta^{*}$. We have regularised all the divergences using dimensional regularisation. The remaining mass singularities are removed by the mass factorisation. To the end, we find

$$
\begin{aligned}
2 S \frac{d \delta \sigma^{P_{1} P_{2}}}{d Q^{2}}\left(\tau, Q^{2}\right)= & \sum_{q} \delta \mathcal{F}_{S M, q} \int_{0}^{1} d x_{1} \int_{0}^{1} d x_{2} \int_{0}^{1} d z \delta\left(\tau-z x_{1} x_{2}\right) \\
& \times\left[\delta H_{q \bar{q}}\left(x_{1}, x_{2}, \mu_{F}^{2}\right)\left(\delta \Delta_{q \bar{q}}^{(0), \gamma Z}\left(z, Q^{2}, \mu_{F}^{2}\right)+a_{s} \delta \Delta_{q \bar{q}}^{(1), \gamma Z}\left(z, Q^{2}, \mu_{F}^{2}\right)\right)\right. \\
& +\delta H_{q g}\left(x_{1}, x_{2}, \mu_{F}^{2}\right)\left(a_{s} \delta \Delta_{q g}^{(1), \gamma Z}\left(z, \mu_{F}^{2}\right)\right) \\
& \left.+\delta H_{g q}\left(x_{1}, x_{2}, \mu_{F}^{2}\right)\left(a_{s} \delta \Delta_{g q}^{(1), \gamma Z}\left(z, \mu_{F}^{2}\right)\right)\right] \\
& +\sum_{q} \delta \mathcal{F}_{G} \int_{0}^{1} d x_{1} \int_{0}^{1} d x_{2} \int_{0}^{1} d z \delta\left(\tau-z x_{1} x_{2}\right) \\
& \times\left[\delta H_{q \bar{q}}\left(x_{1}, x_{2}, \mu_{F}^{2}\right)\left(\delta \Delta_{q \bar{q}}^{(0), G}\left(z, Q^{2}, \mu_{F}^{2}\right)+a_{s} \delta \Delta_{q \bar{q}}^{(1), G}\left(z, Q^{2}, \mu_{F}^{2}\right)\right)\right. \\
& +\delta H_{q g}\left(x_{1}, x_{2}, \mu_{F}^{2}\right)\left(a_{s} \delta \Delta_{q g}^{(1), G}\left(z, \mu_{F}^{2}\right)\right) \\
& \left.\left.+\delta H_{g q}\left(x_{1}, x_{2}, \mu_{F}^{2}\right)\right)\left(a_{s} \delta \Delta_{g q}^{(1), G}\left(z, \mu_{F}^{2}\right)\right)\right] .
\end{aligned}
$$

The constants $\delta \mathcal{F}_{S M, q}, \delta \mathcal{F}_{G}$ are given by

$$
\begin{aligned}
\delta \mathcal{F}_{S M, q}= & 2 \alpha^{2}\left[\frac{\left(Q^{2}-M_{Z}^{2}\right)}{\left(\left(Q^{2}-M_{Z}^{2}\right)^{2}+M_{Z}^{2} \Gamma_{Z}^{2}\right) c_{w}^{2} s_{w}^{2}} Q_{q} Q_{e} g_{q}^{A} g_{e}^{A}\right. \\
& \left.+\frac{2 Q^{2}}{\left(\left(Q^{2}-M_{Z}^{2}\right)^{2}+M_{Z}^{2} \Gamma_{Z}^{2}\right) c_{w}^{4} s_{w}^{4}} g_{q}^{V} g_{e}^{V} g_{q}^{A} g_{e}^{A}\right], \\
\delta \mathcal{F}_{G}= & \frac{\alpha \kappa^{2} Q^{2}}{4 \pi}\left|\mathcal{D}\left(Q^{2}\right)\right|\left[Q_{q} Q_{e}+\frac{Q^{2}\left(Q^{2}-M_{Z}^{2}\right)}{\left(\left(Q^{2}-M_{Z}^{2}\right)^{2}+M_{Z}^{2} \Gamma_{Z}^{2}\right) c_{w}^{2} s_{w}^{2}} g_{q}^{V} g_{e}^{V}\right] .
\end{aligned}
$$


The renormalised incoming partonic fluxes are defined by

$$
\begin{aligned}
& \delta H_{q \bar{q}}\left(x_{1}, x_{2}, \mu_{F}^{2}\right)=f_{q}^{P_{1}}\left(x_{1}, \mu_{F}^{2}\right) f_{\bar{q}}^{P_{2}}\left(x_{2}, \mu_{F}^{2}\right)-f_{\bar{q}}^{P_{1}}\left(x_{1}, \mu_{F}^{2}\right) f_{q}^{P_{2}}\left(x_{2}, \mu_{F}^{2}\right), \\
& \delta H_{g q}\left(x_{1}, x_{2}, \mu_{F}^{2}\right)=f_{g}^{P_{1}}\left(x_{1}, \mu_{F}^{2}\right)\left(f_{q}^{P_{2}}\left(x_{2}, \mu_{F}^{2}\right)-f_{\bar{q}}^{P_{2}}\left(x_{2}, \mu_{F}^{2}\right)\right), \\
& \delta H_{q g}\left(x_{1}, x_{2}, \mu_{F}^{2}\right)=\delta H_{g q}\left(x_{2}, x_{1}, \mu_{F}^{2}\right) .
\end{aligned}
$$

The LO coefficient functions corresponding to Eq. (6.6) are

$$
\begin{aligned}
\delta \Delta_{q \bar{q}}^{(0), \gamma Z} & =\frac{2 \pi}{N} \delta(1-z), \\
\delta \Delta_{q \bar{q}}^{(0), G} & =\frac{\pi}{8 N} \delta(1-z) .
\end{aligned}
$$

The NLO contributions are given by

$$
\begin{aligned}
\delta \Delta_{q \bar{q}}^{(1) \gamma Z} & =\Delta_{q \bar{q}}^{(1) \gamma / Z}+\frac{2 \pi}{N} C_{F}[4(1+z) \ln (z)+4(1-z)], \\
\delta \Delta_{q g}^{(1) \gamma Z} & =\Delta_{q g}^{(1) \gamma / Z}+\frac{2 \pi}{N} T_{f}[4(1-z) \ln (z)], \\
\delta \Delta_{g q}^{(1) \gamma Z} & =-\Delta_{g q}^{(1) \gamma / Z}+\frac{2 \pi}{N} T_{f}\left[8 z^{2} \ln (z)+2\left(1+2 z-3 z^{2}\right)\right], \\
\delta \Delta_{q \bar{q}}^{(1) G} & =\frac{1}{16} \Delta_{q \bar{q}}^{(1) G}+\frac{\pi}{8 N} C_{F}[-4(1+z) \ln (z)-2 \delta(1-z)] \\
\delta \Delta_{q g}^{(1) G} & =\frac{1}{16} \Delta_{q g}^{(1) G}+\frac{\pi}{8 N} T_{f}[-4(1-2 z) \ln (z)+4(1-z)] \\
\delta \Delta_{g q}^{(1) G} & =-\frac{1}{16} \Delta_{g q}^{(1) G}+\frac{\pi}{8 N} T_{f}\left[8\left(2-z+z^{2}\right) \ln (z)+2\left(1+2 z-3 z^{2}\right)\right] .
\end{aligned}
$$

The coefficient functions $\Delta_{a b}^{(1), i}$ with $i=\gamma, Z, G$ correspond to the ones found in the cross section for the invariant lepton pair distribution Eq. (3.33). They can be found in Eq. (3.38). The forward backward asymmetry is defined by

$$
A_{F B}=\left[2 S \frac{d \sigma^{P_{1} P_{2}}}{d Q^{2}}\left(\tau, Q^{2}\right)\right]^{-1} 2 S \frac{d \delta \sigma^{P_{1} P_{2}}}{d Q^{2}}\left(\tau, Q^{2}\right) .
$$

The impact of the NLO correction is discussed in the next section. 


\section{Discussions}

In this section, the effect of the NLO QCD corrections on various distributions such as the invariant mass $Q$, the longitudinal moment fraction $x_{F}$ and the rapidity $Y$ of the lepton pair are presented. We have chosen to present these distributions for the LHC $(\sqrt{S}=14 \mathrm{TeV})$ and for the Run II of Tevatron $(\sqrt{S}=1.96 \mathrm{TeV})$. We want to emphasise that we are not analysing the existing Tevatron data to extract bounds on $M_{S}$, which will require doing a full hadron-level simulation, but are only interested in gauging the impact of the QCD corrections on the bound.

The standard model parameters which enter our analysis are $\alpha=1 / 137.03604$, $M_{Z}=91.1876 \mathrm{GeV}, \Gamma_{Z}=2.4952 \mathrm{GeV}$ and $\sin ^{2} \theta_{W}=0.227$. For the ADD model parameters, we choose $M_{S}=1.5(2) \mathrm{TeV}$ and $d=2(3)$ for the Tevatron (LHC) respectively. For the parton density sets we adopt in leading order MRST 2001 LO $(\Lambda=0.1670 \mathrm{GeV})$ and in next-to-leading order the MRST $2001 \mathrm{NLO}(\Lambda=0.2390$ $\mathrm{GeV})[17]$. For LHC and the Tevatron we choose the range $150 \mathrm{GeV}<Q<1100 \mathrm{GeV}$. Further we have for the LHC the ranges $-0.7<x_{F}<0.7$ and $-2.7<Y<2.7$ at $Q=700 \mathrm{GeV}$. A similar analysis is performed for the Tevatron. Here we choose for the $x_{F}$ and $Y$ distributions the regions $-0.7<x_{F}<0.7$ and $-0.9<Y<0.9$ at $Q=400 \mathrm{GeV}$ respectively. The renormalisation scale $\mu_{R}$ and factorisation scale $\mu_{F}$ are taken to be equal. Finally the factorisation scale $\mu_{F}$ is chosen to be $\mu_{F}=Q$ unless mentioned otherwise.

Let us first study the invariant lepton pair mass distributions

$$
\frac{d \sigma^{I}(Q)}{d Q}
$$

for $I=S M$ (standard model) and $I=G R$ (gravity) and the sum of both mechanisms $S M+G R$.

Starting our discussion with the LHC we have plotted the distribution in Eq. (7.1) for $150 \mathrm{GeV}<Q<1100 \mathrm{GeV}$ in Fig. 10a. The standard model dominates the cross section for $Q<600 \mathrm{GeV}$ but for $Q>600 \mathrm{GeV}$ the graviton mediated process takes over and overwhelms the standard model result. The dominance of the graviton becomes very clear in the longitudinal momentum fraction cross section Fig. 10b

$$
\left.\frac{d \sigma^{I}\left(Q, x_{F}\right)}{d Q d x_{F}}\right|_{Q=700 \mathrm{GeV}},
$$

and the rapidity distribution in Fig. 10c.

$$
\left.\frac{d \sigma^{I}(Q, Y)}{d Q d Y}\right|_{Q=700 \mathrm{GeV}} .
$$

In a large invariant mass bin like $Q=700 \mathrm{GeV}$, the effect of the graviton is clearly discernible in both the $x_{F}$ and $Y$ distributions especially for the central $x_{F}$ and $Y$ 
regions. At large absolute values for both variables the cross section for the graviton mediated processes is again smaller than the standard model result.

In Fig. 11a we have studied the K-factor for the invariant lepton pair mass distribution defined by

$$
K^{I}=\left[\frac{d \sigma_{L O}^{I}(Q)}{d Q}\right]^{-1}\left[\frac{d \sigma_{N L O}^{I}(Q)}{d Q}\right],
$$

where NLO stands for the next-to-leading order corrected cross section. A similar definitions exist for the longitudinal momentum fraction in Fig. 11b

$$
K^{I}=\left.\left[\frac{d \sigma_{L O}^{I}\left(Q, x_{F}\right)}{d Q d x_{F}}\right]^{-1}\left[\frac{d \sigma_{N L O}^{I}\left(Q, x_{F}\right)}{d Q d x_{F}}\right]\right|_{Q=700 \mathrm{GeV}},
$$

and the rapidity in Fig. 11c

$$
K^{I}=\left.\left[\frac{d \sigma_{L O}^{I}(Q, Y)}{d Q d Y}\right]^{-1}\left[\frac{d \sigma_{N L O}^{I}(Q, Y)}{d Q d Y}\right]\right|_{Q=700 \mathrm{GeV}} .
$$

Such a definition is useful for $Q, x_{F}$ and $Y$ distributions because there is no interference between the standard model and the graviton mediated processes. We find that $K^{S M}$ is moderate for all values of $Q$ in Fig. 11a. On the other hand $K^{G R}$ is much larger than the standard model value for $K^{S M}$. In particular for $Q>700 \mathrm{GeV}, K^{G R}$ overwhelms the standard model result $K^{S M}$ completely so that the whole $K^{S M+G R}$ can be attributed to $K^{G R}$. This is because the graviton mediated processes show up already at the Born level (see Eq. (3.35)) and at the LHC the gluon flux is quite large which lead in NLO to big effects mainly due to small $x$ terms in the coefficient function $\Delta_{g g}^{(1) G}$ (Eq. (3.38)). These effects are also visible in Fig. 11b for the $x_{F}$ and in Fig. 11c for the $Y$-distributions. If the choice of parameters $M_{S}=2 \mathrm{TeV}$ and $d=3$ is reasonable then for $Q>700 \mathrm{GeV}$ the effect of the graviton will be observed in view of the cross section which amounts to about $10^{-3}$ pico barn.

In Fig. 12a, Fig. 12b, and Fig. 12c, we have plotted the scale variations of the various distributions for both LO and NLO cross sections. For this we define $R^{I}$ for the invariant lepton mass distribution in Fig. 12a as

$$
\begin{aligned}
R_{L O}^{I} & =\left.\left[\frac{d \sigma_{L O}^{I}\left(Q, \mu=\mu_{0}\right)}{d Q}\right]^{-1}\left[\frac{d \sigma_{L O}^{I}(Q, \mu)}{d Q}\right]\right|_{Q=700 \mathrm{GeV}}, \\
R_{N L O}^{I} & =\left.\left[\frac{d \sigma_{N L O}^{I}\left(Q, \mu=\mu_{0}\right)}{d Q}\right]^{-1}\left[\frac{d \sigma_{N L O}^{I}(Q, \mu)}{d Q}\right]\right|_{Q=700 \mathrm{GeV}},
\end{aligned}
$$


where $\mu_{0}$ is a fixed scale which is chosen to be $\mu_{0}=Q$. For the longitudinal fraction of the lepton pair we have in Fig. 12b

$$
\begin{aligned}
R_{L O}^{I} & =\left.\left[\frac{d \sigma_{L O}^{I}\left(Q, x_{F}, \mu=\mu_{0}\right)}{d Q d x_{F}}\right]^{-1}\left[\frac{d \sigma_{L O}^{I}\left(Q, x_{F}, \mu\right)}{d Q d x_{F}}\right]\right|_{Q=700 \mathrm{GeV}, x_{F}=0}, \\
R_{N L O}^{I} & =\left.\left[\frac{d \sigma_{N L O}^{I}\left(Q, x_{F}, \mu=\mu_{0}\right)}{d Q d x_{F}}\right]^{-1}\left[\frac{d \sigma_{N L O}^{I}\left(Q, x_{F}, \mu\right)}{d Q d x_{F}}\right]\right|_{Q=700 \mathrm{GeV}, x_{F}=0} .
\end{aligned}
$$

Finally we have plotted the scale variation for the rapidity in Fig. 12c

$$
\begin{aligned}
R_{L O}^{I} & =\left.\left[\frac{d \sigma_{L O}^{I}\left(Q, Y, \mu=\mu_{0}\right)}{d Q d Y}\right]^{-1}\left[\frac{d \sigma_{L O}^{I}(Q, \mu)}{d Q d Y}\right]\right|_{Q=700 \mathrm{GeV}, Y=0}, \\
R_{N L O}^{I} & =\left.\left[\frac{d \sigma_{N L O}^{I}\left(Q, Y, \mu=\mu_{0}\right)}{d Q d Y}\right]^{-1}\left[\frac{d \sigma_{N L O}^{I}(Q, \mu)}{d Q d Y}\right]\right|_{Q=700 \mathrm{GeV}, Y=0}
\end{aligned}
$$

In Fig. 12a we observe that for the invariant lepton mass distribution the scale variation is appreciably reduced for $\mu<\mu_{0}$ while going from LO to NLO. For $\mu>\mu_{0}$ the same is happening but here the effect is not very big. However the inclusion of the standard model into gravity makes the situation better in particular for $\mu<\mu_{0}$. This holds for LO as well for NLO. Apparently the gluon initiated processes in the case of the gravity show a larger scale variation than occurs in the case of the standard model. If we look at Eq. $(7.9,7.10)$ plotted in Fig. $12 \mathrm{~b}$ the features are the same. There is an improvement in scale variation while going form LO to NLO. The same holds for Eq. (7.11, 7.12) plotted in Fig. 12c.

Now we turn our attention to the Tevatron. In Fig. 13a we show the result for the invariant mass distribution of the lepton pair. Here the graviton mediated process becomes larger than the standard model result at $Q>700 \mathrm{GeV}$. In the $x_{F}$-distributions (Fig. 13b) and the $Y$-distributions (Fig. 13c) the gravity effects are small. The picture for the $K$-factor differs from the one observed at the LHC. In Fig. 14a the $K$-factor for the graviton is larger than the standard model result at $Q<600 \mathrm{GeV}$. However the value for the cross section is smaller than that of the standard model. Therefore $K^{S M}$ is close to $K^{S M+G R}$. At larger values for $Q$ the cross section for the graviton is larger than the standard model result but $K^{G R}<K^{S M}$ so that also here $K^{S M}$ is close to $K^{S M+G R}$. The last feature is different from that observed for the LHC in Fig. 11a where $K^{S M+G R}>>K^{S M}$. This is because the gluon flux does not dominate the processes at the Tevatron quite contrary to what is observed for the LHC. For the $x_{F}$ in Fig. 14b and $Y$-distributions in Fig. 14c which are taken at $Q=400 \mathrm{GeV}$, the $K^{S M}$ is close to $K^{S M+G R}$. Finally we look at the scale variation in Fig. 15a. For $\mu<\mu_{0}$ there is a better improvement when we go from LO to NLO than that observed 
for the LHC. On the other hand the difference between the graviton mediated process and the graviton plus the standard model becomes less. Also this is an indication that the gluon flux is less important for the Tevatron than for the LHC. The same feature is observed at $Q=400 \mathrm{GeV}$ for the $x_{F}$ distribution in Fig. 15b and the $Y$-distribution in Fig. 15c. Because the full $K$-factor does not differ very much from $K^{S M}$, it may appear that it is sufficient to use the SM K-factor in extracting bounds on $M_{S}$ at the Tevatron. Indeed, existing bounds on $M_{S}$ from the dilepton production process at the Tevatron [18] are obtained using a constant $K$-factor of 1.3. These bounds range from 1.4 to $1 \mathrm{TeV}$ for $\mathrm{d}=2$ to $\mathrm{d}=6$. While the inclusion of the NLO corrections computed here may not change the Tevatron bounds significantly, it will certainly stabilise the cross-section with respect to scale variations. It is for this reason that the inclusion of the NLO corrections becomes important even for the Tevatron.

Finally we consider the forward backward asymmetry defined by

$$
A_{F B}=\left[2 S \frac{d \sigma^{P_{1} P_{2}}}{d Q^{2}}\left(\tau, Q^{2}\right)\right]^{-1} 2 S \frac{d \delta \sigma^{P_{1} P_{2}}}{d Q^{2}}\left(\tau, Q^{2}\right) .
$$

This asymmetry is a result of the interferences of the parity violating structure of $Z$ exchange with the photon [19] and with the graviton initiated process. The dominant contribution to $A_{F B}$ comes from the $q \bar{q}$ subprocess and hence is important at the Tevatron. At LHC for the definition of scattering angle (Eq. 6.2) the $A_{F B}$ will be negligible [19] since the LO contribution vanishes. For the Tevatron the plots are made in Fig. 16 for the parameters $d=2$ and $M_{S}=1.5 \mathrm{TeV}$ in the region $20 \mathrm{GeV}<Q<800$ $\mathrm{GeV}$. Here we have plotted $A_{F B}$ for the SM and the SM+GR at NLO. The effects of extra dimensions show up at $Q>300 \mathrm{GeV}$. In this region the graviton contribution is slightly larger than the SM where the latter shows a constant behaviour.

In summary, we have computed the cross sections $d \sigma / d Q, d^{2} \sigma / d Q d x_{F}, d^{2} \sigma / d Q d Y$ and the forward backward asymmetry $A_{F B}$ up to next to leading order. The standard model result was already given 25 years ago but now we also included all subprocesses due to the graviton in the context of $\mathrm{TeV}$-scale gravity models. Our main conclusion is that the NLO QCD corrections are very significant at the LHC because of the large incident gluon flux at the LHC. At the Tevatron where the gluon flux is small, the NLO effects are also quite small. But, significantly, at both colliders inclusion of the NLO QCD corrections help stabilise the cross-section with respect to scale variations. The extraction of bounds from both colliders will, therefore, require the inclusion of these NLO corrections.

Acknowledgements: VR would like to thank R. K. Ellis for useful discussion. The work of PM and KS is partially supported by the Indo-French Centre for the Promotion of Advanced Research, New Delhi, India (IFCPAR Project No. 2904-2). PM's work is also supported in part by the Commonwealth Scholarship Commission, UK (INCF-2004-92) and Board of Research in Nuclear Sciences, India (BRNS Project No. 2002/37/23). 


\section{Appendix A}

Here we present the $x_{F}$-distributions which were too long to be published in section 4 . The Born contributions (LO) to the coefficient functions are

$$
\begin{aligned}
C_{q \bar{q}}^{S M,(0)}\left(x_{1}^{0}, x_{2}^{0}\right) & =\frac{2 \pi}{N} \frac{H_{q \bar{q}}\left(x_{1}^{0}, x_{2}^{0}, \mu_{F}^{2}\right)}{x_{1}^{0}+x_{2}^{0}}, \\
C_{q \bar{q}}^{G,(0)}\left(x_{1}^{0}, x_{2}^{0}\right) & =\frac{\pi}{8 N} \frac{H_{q \bar{q}}\left(x_{1}^{0}, x_{2}^{0}, \mu_{F}^{2}\right)}{x_{1}^{0}+x_{2}^{0}}, \\
C_{g g}^{G,(0)}\left(x_{1}^{0}, x_{2}^{0}\right) & =\frac{\pi}{2\left(N^{2}-1\right)} \frac{H_{g g}\left(x_{1}^{0}, x_{2}^{0}, \mu_{F}^{2}\right)}{x_{1}^{0}+x_{2}^{0}} .
\end{aligned}
$$

For the NLO corrections we start with processes with a photon or Z-boson in the intermediate state. The corrections are given by [6], [13]-[16]

$$
\begin{aligned}
C_{q \bar{q}}^{S M,(1)}\left(x_{1}^{0}, x_{2}^{0}\right)= & \frac{2 \pi}{N} C_{F}\left\{\int d x _ { 1 } \frac { H _ { q \overline { q } } ( x _ { 1 } , x _ { 2 } ^ { 0 } , \mu _ { F } ^ { 2 } ) } { ( x _ { 1 } ^ { 0 } + x _ { 2 } ^ { 0 } ) } \left[-2 \frac{x_{1}^{0}}{x_{1}^{2}}+\frac{2}{x_{1}}+\left(-2 \frac{x_{1}^{0}}{x_{1}^{2}}-\frac{2}{x_{1}}\right) \mathcal{L}_{a_{1}}\right.\right. \\
& \left.+\frac{4}{\left(x_{1}-x_{1}^{0}\right)} \mathcal{L}_{c_{1}}\right]+\int d x_{1} \frac{H_{q \bar{q}, 1}\left(x_{1}, x_{2}^{0}, \mu_{F}^{2}\right)}{\left(x_{1}-x_{1}^{0}\right)\left(x_{1}^{0}+x_{2}^{0}\right)}\left[4 \mathcal{L}_{b_{1}}\right] \\
& +\int d x_{1} \int d x_{2} \frac{H_{q \bar{q}, 1}\left(x_{1}, x_{2}, \mu_{F}^{2}\right)}{\left(x_{1}-x_{1}^{0}\right)\left(x_{1}^{0}+x_{2}^{0}\right)}\left[-2 \frac{x_{2}^{0}}{x_{2}^{2}}-\frac{2}{x_{2}}\right] \\
& +\int d x_{1} \int d x_{2} \frac{2 H_{q \bar{q}, 12}\left(x_{1}, x_{2}, \mu_{F}^{2}\right)}{\left(x_{1}-x_{1}^{0}\right)\left(x_{2}-x_{2}^{0}\right)\left(x_{1}^{0}+x_{2}^{0}\right)} \\
& +\frac{H_{q \bar{q}}\left(x_{1}^{0}, x_{2}^{0}, \mu_{F}^{2}\right)}{\left(x_{1}^{0}+x_{2}^{0}\right)}\left[\left(3+2 \mathcal{L}\left(x_{1}^{0}, x_{2}^{0}\right)\right) \log \left(\frac{Q^{2}}{\mu_{F}^{2}}\right)+\left(\mathcal{L}\left(x_{1}^{0}, x_{2}^{0}\right)\right)^{2}\right. \\
& +6 \zeta(2)-8] \\
& +\int d x_{1} \int d x_{2} \frac{H_{q \bar{q}}\left(x_{1}, x_{2}, \mu_{F}^{2}\right)}{\left(x_{1}+x_{2}\right)\left(x_{1}+x_{2}^{0}\right)\left(x_{2}+x_{1}^{0}\right)\left(x_{1}^{0}+x_{2}^{0}\right)} \\
& +\left[\frac{2 x_{1}^{0} x_{2}^{0}}{x_{1}^{2}}\left(x_{2}+x_{1}^{0}+x_{2}^{0}\right)+\frac{2}{x_{1}}\left(x_{1}^{0}+x_{1}^{0} x_{2}^{0}-x_{2}^{0}+x_{2}\left(x_{1}^{0}+x_{2}^{0}\right)\right)\right] \\
& +1\}
\end{aligned}
$$




$$
\begin{aligned}
& C_{q g}^{S M,(1)}\left(x_{1}^{0}, x_{2}^{0}\right)= \frac{2 \pi}{N} T_{f}\left\{\int d x_{2} \frac{H_{q g}\left(x_{1}^{0}, x_{2}, \mu_{F}^{2}\right)}{\left(x_{1}^{0}+x_{2}^{0}\right)}\left[4 \frac{x_{2}^{0}}{x_{2}^{2}}-4 \frac{x_{2}^{0^{2}}}{x_{2}^{3}}+\left(\frac{2}{x_{2}}-4 \frac{x_{2}^{0}}{x_{2}^{2}}+4 \frac{x_{2}^{0^{2}}}{x_{2}^{3}}\right) \mathcal{L}_{a_{2}}\right]\right. \\
&+\int d x_{1} \int d x_{2} \frac{H_{q g, 1}\left(x_{1}, x_{2}, \mu_{F}^{2}\right)}{\left(x_{1}-x_{1}^{0}\right)\left(x_{1}^{0}+x_{2}^{0}\right)}\left[\frac{2}{x_{2}}-4 \frac{x_{2}^{0}}{x_{2}^{2}}+4 \frac{x_{2}^{0^{2}}}{x_{2}^{3}}\right] \\
&+\int d x_{1} \int d x_{2} \frac{H_{q g}\left(x_{1}, x_{2}, \mu_{F}^{2}\right)}{\left(x_{1}+x_{2}\right)^{2}\left(x_{1}+x_{2}^{0}\right)\left(x_{1}^{0}+x_{2}^{0}\right)}\left[-\frac{2 x_{1}^{0} x_{2}^{0^{2}}}{x_{1}^{2} x_{2}}\left(x_{1}^{0}+x_{2}^{0}\right)\right. \\
&+\frac{2 x_{2}^{0}}{x_{2}^{2} x_{1}}\left(-2 x_{1}^{0^{2}} x_{2}^{0}+x_{2}^{0} x_{2}\left(2 x_{2}-x_{2}^{0}\right)-x_{1}^{0}\left(-2 x_{2}^{2}+x_{2} x_{2}^{0}+2 x_{2}^{0^{2}}\right)\right) \\
&+\frac{2 x_{1}^{0}}{x_{2}^{3}}\left(x_{2}^{0}\left(5 x_{2}^{2}-2 x_{2} x_{2}^{0}-2 x_{2}^{0^{2}}\right)-x_{1}^{0}\left(x_{2}^{2}-2 x_{2} x_{2}^{0}+2 x_{2}^{0^{2}}\right)\right) \\
&-8 \frac{x_{2}^{0^{3}}}{x_{2}^{2}}+8 \frac{x_{2}^{0^{2}}}{x_{2}}+4 x_{2}^{0}-2 x_{2}+\frac{2 x_{1}}{x_{2}^{3}}\left(x_{1}^{0}\left(x_{2}^{2}+2 x_{2} x_{2}^{0}-2 x_{2}^{0^{2}}\right)\right. \\
&+\left.\left.\left.2 x_{1}^{2}\left(-2 \frac{x_{2}^{0^{2}}}{x_{2}^{3}}+2 \frac{x_{2}^{0}}{x_{2}^{2}}-\frac{1}{x_{2}}\right)-\left(2 x_{2}^{3}-5 x_{2}^{2} x_{2}^{0}+2 x_{2} x_{2}^{0^{2}}+2 x_{2}^{0^{3}}\right)\right)\right]\right\}, \quad(\mathrm{A} .4) \\
&\left.C_{g q}^{S M,(1)}\left(x_{1}^{0}, x_{2}^{0}\right)=\left.C_{q g}^{S M,(1)}\left(x_{1}^{0}, x_{2}^{0}\right)\right|_{1 \leftrightarrow 2} \cdot 3\right)
\end{aligned}
$$

$$
\begin{aligned}
\mathcal{L}_{a_{1}} & =\ln \left(\frac{Q^{2}\left(x_{1}^{0}+x_{2}^{0}\right)\left(1-x_{2}^{0}\right)\left(x_{1}-x_{1}^{0}\right)}{\mu_{F}^{2}\left(x_{1}+x_{2}^{0}\right) x_{1}^{0} x_{2}^{0}}\right), \quad \mathcal{L}_{b_{1}}=\ln \left(\frac{Q^{2}\left(1-x_{2}^{0}\right)\left(x_{1}-x_{1}^{0}\right)}{\mu_{F}^{2} x_{1}^{0} x_{2}^{0}}\right), \\
\mathcal{L}_{c_{1}} & =\ln \left(\frac{x_{1}^{0}+x_{2}^{0}}{x_{1}+x_{2}^{0}}\right), \quad \mathcal{L}\left(x_{1}^{0}, x_{2}^{0}\right)=\ln \left(\frac{\left(1-x_{1}^{0}\right)\left(1-x_{2}^{0}\right)}{x_{1}^{0} x_{2}^{0}}\right) .
\end{aligned}
$$


The $\mathcal{L}_{a_{2}}, \mathcal{L}_{b_{2}}$ and $\mathcal{L}_{c_{2}}$ can be obtained from $\mathcal{L}_{a_{1}}, \mathcal{L}_{b_{1}}$ and $\mathcal{L}_{c_{1}}$ by using $1 \leftrightarrow 2$ symmetry. The NLO corrections with a graviton in the intermediate state are given by

$$
\begin{aligned}
& C_{q \bar{q}}^{G,(1)}\left(x_{1}^{0}, x_{2}^{0}\right)=\frac{\pi}{8 N} C_{F}\left\{\int d x _ { 1 } \frac { H _ { q \overline { q } } ( x _ { 1 } , x _ { 2 } ^ { 0 } , \mu _ { F } ^ { 2 } ) } { ( x _ { 1 } ^ { 0 } + x _ { 2 } ^ { 0 } ) } \left[-2 \frac{x_{1}^{0}}{x_{1}^{2}}+\frac{2}{x_{1}}+\left(-2 \frac{x_{1}^{0}}{x_{1}^{2}}-\frac{2}{x_{1}}\right) \mathcal{L}_{a_{1}}\right.\right. \\
& \left.+\frac{4}{\left(x_{1}-x_{1}^{0}\right)} \mathcal{L}_{c_{1}}\right]+\int d x_{1} \frac{H_{q \bar{q}, 1}\left(x_{1}, x_{2}^{0}, \mu_{F}^{2}\right)}{\left(x_{1}-x_{1}^{0}\right)\left(x_{1}^{0}+x_{2}^{0}\right)}\left[4 \mathcal{L}_{b_{1}}\right] \\
& +\int d x_{1} \int d x_{2} \frac{H_{q \bar{q}, 1}\left(x_{1}, x_{2}, \mu_{F}^{2}\right)}{\left(x_{1}-x_{1}^{0}\right)\left(x_{1}^{0}+x_{2}^{0}\right)}\left[-2 \frac{x_{2}^{0}}{x_{2}^{2}}-\frac{2}{x_{2}}\right] \\
& +\int d x_{1} \int d x_{2} \frac{2 H_{q \bar{q}, 12}\left(x_{1}, x_{2}, \mu_{F}^{2}\right)}{\left(x_{1}-x_{1}^{0}\right)\left(x_{2}-x_{2}^{0}\right)\left(x_{1}^{0}+x_{2}^{0}\right)} \\
& +\frac{H_{q \bar{q}}\left(x_{1}^{0}, x_{2}^{0}, \mu_{F}^{2}\right)}{\left(x_{1}^{0}+x_{2}^{0}\right)}\left[\left(3+2 \mathcal{L}\left(x_{1}^{0}, x_{2}^{0}\right)\right) \log \left(\frac{Q^{2}}{\mu_{F}^{2}}\right)+\left(\mathcal{L}\left(x_{1}^{0}, x_{2}^{0}\right)\right)^{2}\right. \\
& +6 \zeta(2)-10] \\
& +\int d x_{1} \int d x_{2} \frac{H_{q \bar{q}}\left(x_{1}, x_{2}, \mu_{F}^{2}\right)}{\left(x_{1}+x_{2}\right)^{3}\left(x_{1}+x_{2}^{0}\right)\left(x_{2}+x_{1}^{0}\right)\left(x_{1}^{0}+x_{2}^{0}\right)} \\
& \times\left[\frac{1}{x_{1}^{2}}\left(2 x_{2}^{3} x_{1}^{0} x_{2}^{0}+\left(x_{1}^{0}+x_{2}^{0}\right)\left(2 x_{2}^{2} x_{1}^{0} x_{2}^{0}+8 x_{2} x_{1}^{0} x_{2}^{02}+8 x_{1}^{0^{2}} x_{2}^{02}\right)\right)\right. \\
& +\frac{1}{x_{1}}\left(2 x_{2}^{3}\left(x_{1}^{0}+x_{2}^{0}\right)+2 x_{2}^{2}\left(x_{1}^{0^{2}}+11 x_{1}^{0} x_{2}^{0}+7 x_{2}^{0^{2}}\right)\right. \\
& \left.+4 x_{2} x_{2}^{0}\left(11 x_{1}^{0^{2}}+7 x_{1}^{0} x_{2}^{0}-4 x_{2}^{0^{2}}\right)+8 x_{1}^{0} x_{2}^{0}\left(3 x_{1}^{0^{2}}+x_{1}^{0} x_{2}^{0}-2 x_{2}^{0^{2}}\right)\right) \\
& +\frac{8 x_{1}^{3}}{x_{2}^{0}}\left(x_{1}^{0}+x_{2}^{0}\right)+x_{1}^{2}\left(28 x_{1}^{0}-16 \frac{x_{1}^{0^{2}}}{x_{2}^{0}}+44 x_{2}^{0}+x_{2}\left(8-16 \frac{x_{1}^{0}}{x_{2}^{0}}\right.\right. \\
& \left.\left.+24 \frac{x_{2}^{0}}{x_{1}^{0}}\right)\right)+x_{1}\left(-50 x_{1}^{0^{2}}+8 x_{2}^{3}\left(\frac{1}{x_{1}^{0}}+\frac{1}{x_{2}^{0}}\right)+16 \frac{x_{1}^{0^{3}}}{x_{2}^{0}}+8 x_{1}^{0} x_{2}^{0}\right. \\
& \left.+66 x_{2}^{0^{2}}+2 x_{2}\left(-7 x_{1}^{0}+8 \frac{x_{1}^{0^{2}}}{x_{2}^{0}}-7 x_{2}^{0}+8 \frac{x_{2}^{0^{2}}}{x_{1}^{0}}\right)\right)+2\left(8 x_{1}^{0^{3}}-7 x_{1}^{0^{2}} x_{2}^{0}\right.
\end{aligned}
$$




$$
\begin{aligned}
& \left.\left.\left.-7 x_{1}^{0} x_{2}^{0^{2}}+8 x_{2}^{0^{3}}\right)\right]+1 \leftrightarrow 2\right\} \\
& C_{g g}^{G,(1)}\left(x_{1}^{0}, x_{2}^{0}\right)=\frac{\pi}{2\left(N^{2}-1\right)} C_{A}\left\{\int d x _ { 1 } \frac { H _ { g g } ( x _ { 1 } , x _ { 2 } ^ { 0 } , \mu _ { F } ^ { 2 } ) } { ( x _ { 1 } ^ { 0 } + x _ { 2 } ^ { 0 } ) } \left[\left(-4 \frac{x_{1}^{0^{2}}}{x_{1}^{3}}+4 \frac{x_{1}^{0}}{x_{1}^{2}}-\frac{8}{x_{1}}+\frac{4}{x_{1}^{0}}\right) \mathcal{L}_{a_{1}}\right.\right. \\
& \left.+\frac{4}{\left(x_{1}-x_{1}^{0}\right)} \mathcal{L}_{c_{1}}\right]+\int d x_{1} \frac{H_{g g, 1}\left(x_{1}, x_{2}^{0}, \mu_{F}^{2}\right)}{\left(x_{1}-x_{1}^{0}\right)\left(x_{1}^{0}+x_{2}^{0}\right)}\left[4 \mathcal{L}_{b_{1}}\right] \\
& +\int d x_{1} \int d x_{2} \frac{H_{g g, 1}\left(x_{1}, x_{2}, \mu_{F}^{2}\right)}{\left(x_{1}-x_{1}^{0}\right)\left(x_{1}^{0}+x_{2}^{0}\right)}\left[\left(-4 \frac{x_{2}^{0^{2}}}{x_{2}^{3}}+4 \frac{x_{2}^{0}}{x_{2}^{2}}-\frac{8}{x_{2}}+\frac{4}{x_{2}^{0}}\right)\right] \\
& +\int d x_{1} \int d x_{2} \frac{2 H_{g g, 12}\left(x_{1}, x_{2}, \mu_{F}^{2}\right)}{\left(x_{1}-x_{1}^{0}\right)\left(x_{2}-x_{2}^{0}\right)\left(x_{1}^{0}+x_{2}^{0}\right)} \\
& +\frac{H_{g g}\left(x_{1}^{0}, x_{2}^{0}, \mu_{F}^{2}\right)}{\left(x_{1}^{0}+x_{2}^{0}\right)}\left[\left(\frac{11}{3}+2 \mathcal{L}\left(x_{1}^{0}, x_{2}^{0}\right)\right) \log \left(\frac{Q^{2}}{\mu_{F}^{2}}\right)+\left(\mathcal{L}\left(x_{1}^{0}, x_{2}^{0}\right)\right)^{2}\right. \\
& \left.\left.+6 \zeta(2)-\frac{203}{18}\right]\right\} \\
& +\frac{n_{f} T_{f} H_{g g}\left(x_{1}^{0}, x_{2}^{0}, \mu_{F}^{2}\right)}{\left(x_{1}^{0}+x_{2}^{0}\right)}\left[-\frac{4}{3} \log \left(\frac{Q^{2}}{\mu_{F}^{2}}\right)+\frac{35}{9}\right] \\
& +C_{A}\left\{\int d x_{1} \int d x_{2} \frac{H_{g g}\left(x_{1}, x_{2}, \mu_{F}^{2}\right)}{\left(x_{1}+x_{2}\right)^{3}\left(x_{1}+x_{2}^{0}\right)\left(x_{2}+x_{1}^{0}\right)\left(x_{1}^{0}+x_{2}^{0}\right)}\right. \\
& \times\left[\frac{1}{x_{1}^{3}}\left(4 x_{2}^{3} x_{1}^{0^{2}} x_{2}^{0}+4 x_{2}^{2}\left(x_{1}^{0^{2}} x_{2}^{0^{2}}+x_{1}^{0^{3}} x_{2}^{0}\right)+4 x_{2}\left(x_{1}^{0^{2}} x_{2}^{0^{3}}+x_{1}^{0^{3}} x_{2}^{0^{2}}\right)\right)\right. \\
& +\frac{1}{x_{1}^{2}}\left(4 x_{2}^{3}\left(x_{1}^{0^{2}}-x_{1}^{0} x_{2}^{0}\right)+x_{2}^{2}\left(4 x_{1}^{0^{3}}+12 x_{1}^{0^{2}} x_{2}^{0}-4 x_{1}^{0} x_{2}^{0^{2}}\right)\right. \\
& \left.+x_{2}\left(-4 x_{1}^{0} x_{2}^{0^{3}}+16 x_{1}^{0^{3}} x_{2}^{0}+12 x_{1}^{0^{2}} x_{2}^{0^{2}}\right)+8 x_{1}^{0^{2}} x_{2}^{0^{3}}+8 x_{1}^{0^{3}} x_{2}^{0^{2}}\right) \\
& +\frac{1}{x_{1}}\left(x_{2}^{3}\left(-4 x_{1}^{0}+8 x_{2}^{0}\right)+x_{2}\left(-32 x_{1}^{0} x_{2}^{0^{2}}-16 x_{1}^{0^{2}} x_{2}^{0}+12 x_{1}^{0^{3}}+8 x_{2}^{0^{3}}\right)\right.
\end{aligned}
$$




$$
\begin{aligned}
& +x_{2}^{2}\left(-32 x_{1}^{0} x_{2}^{0}+8 x_{1}^{0^{2}}-16 x_{2}^{0^{2}}\right)+\frac{1}{2 x_{2}}\left(12 x_{1}^{0^{2}} x_{2}^{0^{3}}+12 x_{1}^{0^{3}} x_{2}^{0^{2}}\right) \\
& \left.+8 x_{1}^{0} x_{2}^{0^{3}}+20 x_{1}^{0^{2}} x_{2}^{0^{2}}+12 x_{1}^{0^{3}} x_{2}^{0}\right)+\frac{4 x_{1}^{4}}{x_{2}^{0}}+x_{1}^{3}\left(12-8 \frac{x_{1}^{0}}{x_{2}^{0}}\right) \\
& +x_{1}^{2}\left(4 \frac{x_{1}^{0^{2}}}{x_{2}^{0}}-28 x_{2}^{0}\right)+x_{1}\left(-64 x_{1}^{0} x_{2}^{0}-12 x_{1}^{0^{2}}-\frac{4 x_{1}^{0^{3}}}{x_{2}^{0}}-24 x_{2}^{0^{2}}\right. \\
& +x_{2}\left(-4 \frac{x_{2}^{0^{2}}}{x_{1}^{0}}-4 x_{1}^{0}-4 \frac{x_{1}^{0^{2}}}{x_{2}^{0}}-4 x_{2}^{0}\right)+x_{2}^{2}\left(-8 \frac{x_{2}^{0}}{x_{1}^{0}}-12 \frac{x_{1}^{0}}{x_{2}^{0}}+40\right) \\
& \left.\left.\left.+4 x_{2}^{3}\left(\frac{1}{x_{1}^{0}}-\frac{1}{x_{2}^{0}}\right)\right)-14 x_{1}^{0} x_{2}^{0^{2}}-14 x_{1}^{0^{2}} x_{2}^{0}+2 x_{1}^{0^{3}}+2 x_{2}^{0^{3}}\right]+1 \leftrightarrow 2\right\}, \\
& C_{q g}^{G,(1)}\left(x_{1}^{0}, x_{2}^{0}\right)=\frac{\pi}{8 N} T_{f}\left\{\int d x_{2} \frac{H_{q g}\left(x_{1}^{0}, x_{2}, \mu_{F}^{2}\right)}{\left(x_{1}^{0}+x_{2}^{0}\right)}\left[4 \frac{x_{2}^{0}}{x_{2}^{2}}-4 \frac{x_{2}^{0^{2}}}{x_{2}^{3}}+\left(\frac{2}{x_{2}}-4 \frac{x_{2}^{0}}{x_{2}^{2}}+4 \frac{x_{2}^{0^{2}}}{x_{2}^{3}}\right) \mathcal{L}_{a_{2}}\right]\right. \\
& +\int d x_{1} \int d x_{2} \frac{H_{q g, 1}\left(x_{1}, x_{2}, \mu_{F}^{2}\right)}{\left(x_{1}-x_{1}^{0}\right)\left(x_{1}^{0}+x_{2}^{0}\right)}\left[\frac{2}{x_{2}}-4 \frac{x_{2}^{0}}{x_{2}^{2}}+4 \frac{x_{2}^{0^{2}}}{x_{2}^{3}}\right] \\
& +\int d x_{1} \frac{H_{q g}\left(x_{1}, x_{2}^{0}, \mu_{F}^{2}\right)}{\left(x_{1}^{0}+x_{2}^{0}\right)}\left[8 \frac{x_{1}^{0}}{x_{1}^{2}}+\left(8 \frac{x_{1}^{0}}{x_{1}^{2}}-\frac{16}{x_{1}}+\frac{16}{x_{1}^{0}}\right) \mathcal{L}_{a_{1}}\right] \\
& +\int d x_{1} \int d x_{2} \frac{H_{q g, 2}\left(x_{1}, x_{2}, \mu_{F}^{2}\right)}{\left(x_{2}-x_{2}^{0}\right)\left(x_{1}^{0}+x_{2}^{0}\right)}\left[8 \frac{x_{1}^{0}}{x_{1}^{2}}-\frac{16}{x_{1}}+\frac{16}{x_{1}^{0}}\right] \\
& +\int d x_{1} \int d x_{2} \frac{H_{q g}\left(x_{1}, x_{2}, \mu_{F}^{2}\right)}{\left(x_{1}+x_{2}\right)^{2}\left(x_{1}+x_{2}^{0}\right)\left(x_{2}+x_{1}^{0}\right)\left(x_{1}^{0}+x_{2}^{0}\right)} \\
& \times\left[\frac { 1 } { x _ { 1 } ^ { 2 } } \left(-2 x_{1}^{0} x_{2}^{0^{3}}-2 x_{1}^{0^{2}} x_{2}^{0^{2}}+x_{2}^{2}\left(-8 x_{1}^{0} x_{2}^{0}\right)\right.\right. \\
& \left.+x_{2}\left(-8 x_{1}^{0^{2}} x_{2}^{0}-8 x_{1}^{0} x_{2}^{0^{2}}\right)+\frac{1}{x_{2}}\left(-2 x_{1}^{0^{3}} x_{2}^{0^{2}}-2 x_{1}^{0^{2}} x_{2}^{0^{3}}\right)\right) \\
& +\frac{1}{x_{1}}\left(-2 x_{2}^{0^{3}}-12 x_{1}^{0^{2}} x_{2}^{0}-14 x_{1}^{0} x_{2}^{0^{2}}+x_{2}^{2}\left(16 x_{2}^{0}-8 x_{1}^{0}\right)\right.
\end{aligned}
$$




$$
\begin{aligned}
& +x_{2}\left(4 x_{2}^{0^{2}}-8 x_{1}^{0^{2}}-20 x_{1}^{0} x_{2}^{0}\right)+\frac{1}{x_{2}}\left(-6 x_{1}^{0^{2}} x_{2}^{0^{2}}-6 x_{1}^{0} x_{2}^{0^{3}}\right) \\
& \left.+\frac{1}{x_{2}^{2}}\left(-4 x_{1}^{0^{3}} x_{2}^{0^{2}}-4 x_{1}^{0^{2}} x_{2}^{0^{3}}\right)\right)+\frac{16 x_{1}^{3}}{x_{2}^{0}}+x_{1}^{2}\left(14-16 \frac{x_{1}^{0}}{x_{2}^{0}}\right. \\
& +x_{2}\left(\frac{16}{x_{2}^{0}}-\frac{16}{x_{1}^{0}}\right)+\frac{1}{x_{2}}\left(4 x_{2}^{0}-2 x_{1}^{0}\right)+\frac{1}{x_{2}^{2}}\left(-4 x_{2}^{0^{2}}+4 x_{1}^{0} x_{2}^{0}\right) \\
& \left.+\frac{1}{x_{2}^{3}}\left(-4 x_{1}^{0} x_{2}^{0^{2}}\right)\right)+x_{1}\left(-6 x_{2}^{0}+8 \frac{x_{1}^{0^{2}}}{x_{2}^{0}}-34 x_{1}^{0}+x_{2}^{2}\left(\frac{8}{x_{2}^{0}}-\frac{8}{x_{1}^{0}}\right)\right. \\
& +x_{2}\left(36-24 \frac{x_{2}^{0}}{x_{1}^{0}}\right)+\frac{1}{x_{2}}\left(-4 x_{2}^{0^{2}}-14 x_{1}^{0^{2}}-2 x_{1}^{0} x_{2}^{0}\right) \\
& \left.+\frac{1}{x_{2}^{2}}\left(-4 x_{2}^{0^{3}}+4 x_{1}^{0^{2}} x_{2}^{0}-8 x_{1}^{0} x_{2}^{0^{2}}\right)+\frac{1}{x_{2}^{3}}\left(-4 x_{1}^{0^{2}} x_{2}^{0^{2}}-4 x_{1}^{0} x_{2}^{0^{3}}\right)\right) \\
& +x_{2}^{2}\left(22-8 \frac{x_{2}^{0}}{x_{1}^{0}}\right)+x_{2}\left(28 x_{2}^{0}-18 x_{1}^{0}-8 \frac{x_{2}^{0^{2}}}{x_{1}^{0}}\right)+\frac{1}{x_{2}}\left(-8 x_{2}^{0^{3}}\right. \\
& \left.\left.+\frac{1}{x_{2}^{3}}\left(-4 x_{1}^{0^{3}} x_{2}^{0^{2}}-4 x_{1}^{0^{2}} x_{2}^{0^{3}}\right)-8 x_{2}^{0^{2}}-10 x_{1}^{0^{2}}-18 x_{1}^{0} x_{2}^{0}\right]\right\} \\
& \left.-\left.2 x_{1 g}^{G}\left(x_{1}^{0}, x_{2}^{0}\right)\right|_{1 \leftrightarrow 2} \cdot 2 x_{1}^{0^{2}} x_{2}^{0}-12 x_{1}^{0} x_{2}^{0^{2}}\right)+\frac{1}{x_{2}^{2}}\left(4 x_{1}^{0^{3}} x_{2}^{0}-8 x_{1}^{0^{2}} x_{2}^{0^{2}}-12 x_{1}^{0} x_{2}^{0^{3}}\right)
\end{aligned}
$$




\section{Appendix B}

Here we present the $Y$-distributions which were too long to be published in section 5 . The Born contributions (LO) to the coefficient functions are

$$
\begin{aligned}
D_{q \bar{q}}^{S M,(0)}\left(x_{1}^{0}, x_{2}^{0}\right) & =\frac{2 \pi}{N} H_{q \bar{q}}\left(x_{1}^{0}, x_{2}^{0}, \mu_{F}^{2}\right), \\
D_{q \bar{q}}^{G,(0)}\left(x_{1}^{0}, x_{2}^{0}\right) & =\frac{\pi}{8 N} H_{q \bar{q}}\left(x_{1}^{0}, x_{2}^{0}, \mu_{F}^{2}\right), \\
D_{g g}^{G,(0)}\left(x_{1}^{0}, x_{2}^{0}\right) & =\frac{\pi}{2\left(N^{2}-1\right)} H_{g g}\left(x_{1}^{0}, x_{2}^{0}, \mu_{F}^{2}\right) .
\end{aligned}
$$

For the NLO corrections we start with processes with a photon or Z-boson in the intermediate state. The corrections are given by [6], [13]-[16]

$$
\begin{aligned}
& D_{q \bar{q}}^{S M,(1)}\left(x_{1}^{0}, x_{2}^{0}\right)=\frac{2 \pi}{N} C_{F}\left\{\int d x _ { 1 } H _ { q \overline { q } } ( x _ { 1 } , x _ { 2 } ^ { 0 } , \mu _ { F } ^ { 2 } ) \left[-2 \frac{x_{1}^{0}}{x_{1}^{2}}+\frac{2}{x_{1}}+\left(-2 \frac{x_{1}^{0}}{x_{1}^{2}}-\frac{2}{x_{1}}\right) \mathcal{K}_{a_{1}}\right.\right. \\
& \left.+\frac{4}{\left(x_{1}-x_{1}^{0}\right)} \mathcal{K}_{c_{1}}\right]+\int d x_{1} \frac{H_{q \bar{q}, 1}\left(x_{1}, x_{2}^{0}, \mu_{F}^{2}\right)}{\left(x_{1}-x_{1}^{0}\right)}\left[4 \mathcal{K}_{b_{1}}\right] \\
& +\int d x_{1} \int d x_{2} \frac{H_{q \bar{q}, 1}\left(x_{1}, x_{2}, \mu_{F}^{2}\right)}{\left(x_{1}-x_{1}^{0}\right)}\left[-2 \frac{x_{2}^{0}}{x_{2}^{2}}-\frac{2}{x_{2}}\right] \\
& +\int d x_{1} \int d x_{2} \frac{2 H_{q \bar{q}, 12}\left(x_{1}, x_{2}, \mu_{F}^{2}\right)}{\left(x_{1}-x_{1}^{0}\right)\left(x_{2}-x_{2}^{0}\right)} \\
& +H_{q \bar{q}}\left(x_{1}^{0}, x_{2}^{0}, \mu_{F}^{2}\right)\left[\left(3+2 \mathcal{K}\left(x_{1}^{0}, x_{2}^{0}\right)\right) \log \left(\frac{Q^{2}}{\mu_{F}^{2}}\right)+\left(\mathcal{K}\left(x_{1}^{0}, x_{2}^{0}\right)\right)^{2}\right. \\
& +6 \zeta(2)-8] \\
& +\int d x_{1} \int d x_{2} \frac{H_{q \bar{q}}\left(x_{1}, x_{2}, \mu_{F}^{2}\right)}{\left(x_{1}+x_{1}^{0}\right)\left(x_{2}+x_{2}^{0}\right)\left(x_{1} x_{2}^{0}+x_{1}^{0} x_{2}\right)^{2}}\left[\frac { 1 } { x _ { 1 } ^ { 2 } } \left(4 x_{1}^{0^{4}} x_{2}^{0^{2}}\right.\right. \\
& \left.+x_{2}^{2}\left(2 x_{1}^{0^{4}}\right)+x_{2}\left(4 x_{1}^{0^{4}} x_{2}^{0}\right)\right)+\frac{1}{x_{1}}\left(4 x_{1}^{0^{3}} x_{2}^{0^{2}}+x_{2}^{2}\left(4 x_{1}^{0^{3}}\right)+x_{2}\left(12 x_{1}^{0^{3}} x_{2}^{0}\right)\right) \\
& \left.\left.+x_{1}\left(4 x_{1}^{0} x_{2}^{0^{2}}\right)+x_{1}^{2}\left(4 x_{2}^{0^{2}}\right)+2 x_{1}^{0^{2}} x_{2}^{0^{2}}\right]+1 \leftrightarrow 2\right\}
\end{aligned}
$$




$$
\begin{aligned}
D_{q g}^{S M,(1)}\left(x_{1}^{0}, x_{2}^{0}\right)= & \frac{2 \pi}{N} T_{f}\left\{\int d x _ { 2 } H _ { q g } ( x _ { 1 } ^ { 0 } , x _ { 2 } , \mu _ { F } ^ { 2 } ) \left[4 \frac{x_{2}^{0}}{x_{2}^{2}}-4 \frac{x_{2}^{0^{2}}}{x_{2}^{3}}+\left(\frac{2}{x_{2}}-4 \frac{x_{2}^{0}}{x_{2}^{2}}+4 \frac{x_{2}^{0^{2}}}{x_{2}^{3}}\right) \mathcal{K}_{a_{2}}\right.\right. \\
& +\int d x_{1} \int d x_{2} \frac{H_{q g, 1}\left(x_{1}, x_{2}, \mu_{F}^{2}\right)}{\left(x_{1}-x_{1}^{0}\right)}\left[\frac{2}{x_{2}}-4 \frac{x_{2}^{0}}{x_{2}^{2}}+4 \frac{x_{2}^{0^{2}}}{x_{2}^{3}}\right] \\
& +\int d x_{1} \int d x_{2} \frac{H_{q g}\left(x_{1}, x_{2}, \mu_{F}^{2}\right)}{\left(x_{1}+x_{1}^{0}\right)\left(x_{1} x_{2}^{0}+x_{1}^{0} x_{2}\right)^{3}}\left[\frac{1}{x_{1}^{2}}\left(-4 \frac{x_{1}^{0^{5}} x_{2}^{0^{3}}}{x_{2}}\right)\right. \\
& +\frac{1}{x_{1}}\left(4 x_{1}^{0^{4}} x_{2}^{0^{2}}-4 \frac{x_{1}^{0^{4}} x_{2}^{0^{3}}}{x_{2}}-8 \frac{x_{1}^{0^{4}} x_{2}^{0^{4}}}{x_{2}^{2}}\right)+x_{1}^{3}\left(-2 \frac{x_{2}^{0^{3}}}{x_{2}}+4 \frac{x_{2}^{0^{4}}}{x_{2}^{2}}\right. \\
& \left.-4 \frac{x_{2}^{0^{5}}}{x_{2}^{3}}\right)+x_{1}^{2}\left(2 x_{1}^{0} x_{2}^{0^{2}}+8 \frac{x_{1}^{0} x_{2}^{0^{3}}}{x_{2}}-4 \frac{x_{1}^{0} x_{2}^{0^{4}}}{x_{2}^{2}}-8 \frac{x_{1}^{0} x_{2}^{0^{5}}}{x_{2}^{3}}\right) \\
& +x_{1}\left(8 x_{1}^{0^{2}} x_{2}^{0^{2}}+2 x_{2} x_{1}^{0^{2}} x_{2}^{0}+16 \frac{x_{1}^{0^{2}} x_{2}^{0^{3}}}{x_{2}}-16 \frac{x_{1}^{0^{2}} x_{2}^{0^{4}}}{x_{2}^{2}}-8 \frac{x_{1}^{0^{2}} x_{2}^{0^{5}}}{x_{2}^{3}}\right) \\
& \left.\left.+8 x_{1}^{0^{3}} x_{2}^{0^{2}}+2 x_{2}^{2} x_{1}^{0^{3}}+4 \frac{x_{1}^{0^{3}} x_{2}^{0^{3}}}{x_{2}}-8 \frac{x_{1}^{0^{3}} x_{2}^{0^{4}}}{x_{2}^{2}}-8 \frac{x_{1}^{0^{3}} x_{2}^{0^{5}}}{x_{2}^{3}}\right]\right\} \\
D_{g q}^{S M,(1)}\left(x_{1}^{0}, x_{2}^{0}\right)= & \left.D_{q g}^{S M}\left(x_{1}^{0}, x_{2}^{0}\right)\right|_{1 \leftrightarrow 2} \cdot(\mathrm{B} .4)
\end{aligned}
$$

We have introduced the following abbreviations

$$
\begin{aligned}
& \mathcal{K}_{a_{1}}=\ln \left(\frac{2 Q^{2}\left(1-x_{2}^{0}\right)\left(x_{1}-x_{1}^{0}\right)}{\mu_{F}^{2}\left(x_{1}+x_{1}^{0}\right) x_{2}^{0}}\right), \quad \mathcal{K}_{b_{1}}=\ln \left(\frac{Q^{2}\left(1-x_{2}^{0}\right)\left(x_{1}-x_{1}^{0}\right)}{\mu_{F}^{2} x_{1}^{0} x_{2}^{0}}\right), \\
& \mathcal{K}_{c_{1}}=\ln \left(\frac{2 x_{1}^{0}}{x_{1}+x_{1}^{0}}\right), \quad \mathcal{K}\left(x_{1}^{0}, x_{2}^{0}\right)=\ln \left(\frac{\left(1-x_{1}^{0}\right)\left(1-x_{2}^{0}\right)}{x_{1}^{0} x_{2}^{0}}\right) .
\end{aligned}
$$

The $\mathcal{K}_{a_{2}}, \mathcal{K}_{b_{2}}$ and $\mathcal{K}_{c_{2}}$ can be obtained from $\mathcal{K}_{a_{1}}, \mathcal{K}_{b_{1}}$ and $\mathcal{K}_{c_{1}}$ by using $1 \leftrightarrow 2$ symmetry. The NLO corrections with a graviton in the intermediate state are given by

$$
\begin{aligned}
D_{q \bar{q}}^{G,(1)}\left(x_{1}^{0}, x_{2}^{0}\right)= & \frac{\pi}{8 N} C_{F}\left\{\int d x _ { 1 } H _ { q \overline { q } } ( x _ { 1 } , x _ { 2 } ^ { 0 } ) \left[-2 \frac{x_{1}^{0}}{x_{1}^{2}}+\frac{2}{x_{1}}+\left(-2 \frac{x_{1}^{0}}{x_{1}^{2}}-\frac{2}{x_{1}}\right) \mathcal{K}_{a_{1}}\right.\right. \\
& \left.+\frac{4}{\left(x_{1}-x_{1}^{0}\right)} \mathcal{K}_{c_{1}}\right]+\int d x_{1} \frac{H_{q \bar{q}, 1}\left(x_{1}, x_{2}^{0}, \mu_{F}^{2}\right)}{\left(x_{1}-x_{1}^{0}\right)}\left[4 \mathcal{K}_{b_{1}}\right] \\
& +\int d x_{1} \int d x_{2} \frac{H_{q \bar{q}, 1}\left(x_{1}, x_{2}, \mu_{F}^{2}\right)}{\left(x_{1}-x_{1}^{0}\right)}\left[-2 \frac{x_{2}^{0}}{x_{2}^{2}}-\frac{2}{x_{2}}\right]
\end{aligned}
$$




$$
\begin{aligned}
& +\int d x_{1} \int d x_{2} \frac{2 H_{q \bar{q}, 12}\left(x_{1}, x_{2}, \mu_{F}^{2}\right)}{\left(x_{1}-x_{1}^{0}\right)\left(x_{2}-x_{2}^{0}\right)} \\
& +H_{q \bar{q}}\left(x_{1}^{0}, x_{2}^{0}, \mu_{F}^{2}\right)\left[\left(3+2 \mathcal{K}\left(x_{1}^{0}, x_{2}^{0}\right)\right) \log \left(\frac{Q^{2}}{\mu_{F}^{2}}\right)+\left(\mathcal{K}\left(x_{1}^{0}, x_{2}^{0}\right)\right)^{2}\right. \\
& +6 \zeta(2)-10] \\
& +\int d x_{1} \int d x_{2} \frac{H_{q \bar{q}}\left(x_{1}, x_{2}, \mu_{F}^{2}\right)}{\left(x_{1}+x_{1}^{0}\right)\left(x_{2}+x_{2}^{0}\right)\left(x_{1} x_{2}^{0}+x_{1}^{0} x_{2}\right)^{4}}\left[\frac { 1 } { x _ { 1 } ^ { 2 } } \left(16 x_{1}^{0^{6}} x_{2}^{0^{4}}\right.\right. \\
& \left.+2 x_{2}^{4} x_{1}^{0^{6}}+4 x_{2}^{3} x_{1}^{0^{6}} x_{2}^{0}+4 x_{2}^{2} x_{1}^{0^{6}} x_{2}^{0^{2}}+16 x_{2} x_{1}^{0^{6}} x_{2}^{0^{3}}\right) \\
& +\frac{1}{x_{1}}\left(16 x_{1}^{0^{5}} x_{2}^{0^{4}}+4 x_{2}^{4} x_{1}^{0^{5}}+16 x_{2}^{3} x_{1}^{0^{5}} x_{2}^{0}+60 x_{2}^{2} x_{1}^{0^{5}} x_{2}^{0^{2}}+72 x_{2} x_{1}^{0^{5}} x_{2}^{0^{3}}\right) \\
& +4 x_{1}^{4} x_{2}^{0^{4}}+60 x_{1}^{3} x_{1}^{0} x_{2}^{0^{4}}+x_{1}^{2}\left(126 x_{1}^{0^{2}} x_{2}^{0^{4}}+16 x_{2}^{4} x_{1}^{0^{2}}+16 x_{2}^{3} x_{1}^{0^{2}} x_{2}^{0}\right. \\
& \left.+4 x_{2}^{2} x_{1}^{0^{2}} x_{2}^{0^{2}}\right)+x_{1}\left(64 x_{1}^{0^{3}} x_{2}^{0^{4}}+16 x_{2}^{4} x_{1}^{0^{3}}+72 x_{2}^{3} x_{1}^{0^{3}} x_{2}^{0}+64 x_{2}^{2} x_{1}^{0^{3}} x_{2}^{0^{2}}\right. \\
& \left.\left.\left.+16 x_{2} x_{1}^{0^{3}} x_{2}^{0^{3}}\right)+4 x_{1}^{0^{4}} x_{2}^{0^{4}}\right]+1 \leftrightarrow 2\right\} \\
& D_{g g}^{G,(1)}\left(x_{1}^{0}, x_{2}^{0}\right)=\frac{\pi}{2\left(N^{2}-1\right)} C_{A}\left\{\int d x _ { 1 } H _ { g g } ( x _ { 1 } , x _ { 2 } ^ { 0 } , \mu _ { F } ^ { 2 } ) \left[\left(-4 \frac{x_{1}^{0^{2}}}{x_{1}^{3}}+4 \frac{x_{1}^{0}}{x_{1}^{2}}-\frac{8}{x_{1}}+\frac{4}{x_{1}^{0}}\right) \mathcal{K}_{a_{1}}\right.\right. \\
& \left.+\frac{4}{\left(x_{1}-x_{1}^{0}\right)} \mathcal{K}_{c_{1}}\right]+\int d x_{1} \frac{H_{g g, 1}\left(x_{1}, x_{2}^{0}, \mu_{F}^{2}\right)}{\left(x_{1}-x_{1}^{0}\right)}\left[4 \mathcal{K}_{b_{1}}\right] \\
& +\int d x_{1} \int d x_{2} \frac{H_{g g, 1}\left(x_{1}, x_{2}, \mu_{F}^{2}\right)}{\left(x_{1}-x_{1}^{0}\right)}\left[\left(-4 \frac{x_{2}^{0^{2}}}{x_{2}^{3}}+4 \frac{x_{2}^{0}}{x_{2}^{2}}-\frac{8}{x_{2}}+\frac{4}{x_{2}^{0}}\right)\right] \\
& +\int d x_{1} \int d x_{2} \frac{2 H_{g g, 12}\left(x_{1}, x_{2}, \mu_{F}^{2}\right)}{\left(x_{1}-x_{1}^{0}\right)\left(x_{2}-x_{2}^{0}\right)} \\
& +H_{g g}\left(x_{1}^{0}, x_{2}^{0}, \mu_{F}^{2}\right)\left[\left(\frac{11}{3}+2 \mathcal{K}\left(x_{1}^{0}, x_{2}^{0}\right)\right) \log \left(\frac{Q^{2}}{\mu_{F}^{2}}\right)+\left(\mathcal{K}\left(x_{1}^{0}, x_{2}^{0}\right)\right)^{2}\right.
\end{aligned}
$$




$$
\begin{aligned}
& \left.\left.+6 \zeta(2)-\frac{203}{18}\right]\right\} \\
& +n_{f} T_{f} H_{g g}\left(x_{1}^{0}, x_{2}^{0}, \mu_{F}^{2}\right)\left[-\frac{4}{3} \log \left(\frac{Q^{2}}{\mu_{F}^{2}}\right)+\frac{35}{9}\right] \\
& +C_{A}\left\{\int d x _ { 1 } \int d x _ { 2 } \frac { H _ { g g } ( x _ { 1 } , x _ { 2 } , \mu _ { F } ^ { 2 } ) } { ( x _ { 1 } + x _ { 1 } ^ { 0 } ) ( x _ { 2 } + x _ { 2 } ^ { 0 } ) ( x _ { 1 } x _ { 2 } ^ { 0 } + x _ { 1 } ^ { 0 } x _ { 2 } ) ^ { 4 } } \left[\frac { 1 } { x _ { 1 } ^ { 3 } } \left(4 x_{2}^{4} x_{1}^{0^{7}}\right.\right.\right. \\
& \left.+8 x_{2}^{3} x_{1}^{0^{7}} x_{2}^{0}+8 x_{2}^{2} x_{1}^{0^{7}} x_{2}^{0^{2}}+8 x_{2} x_{1}^{0^{7}} x_{2}^{0^{3}}\right)+\frac{1}{x_{1}^{2}}\left(16 x_{1}^{0^{6}} x_{2}^{0^{4}}+16 x_{2}^{3} x_{1}^{0^{6}} x_{2}^{0}\right. \\
& \left.+32 x_{2}^{2} x_{1}^{0^{6}} x_{2}^{0^{2}}+24 x_{2} x_{1}^{0^{6}} x_{2}^{0^{3}}\right)+\frac{1}{x_{1}}\left(40 x_{1}^{0^{5}} x_{2}^{0^{4}}+4 x_{2}^{4} x_{1}^{0^{5}}+8 x_{2}^{3} x_{1}^{0^{5}} x_{2}^{0}\right. \\
& \left.-16 x_{2}^{2} x_{1}^{0^{5}} x_{2}^{0^{2}}+12 \frac{x_{1}^{0^{5}} x_{2}^{0^{5}}}{x_{2}}\right)+4 \frac{x_{1}^{5} x_{2}^{0^{4}}}{x_{1}^{0}}+4 x_{1}^{4} x_{2}^{0^{4}}+x_{1}^{3}\left(-20 x_{1}^{0} x_{2}^{0^{4}}\right. \\
& \left.+12 x_{2}^{3} x_{1}^{0} x_{2}^{0}\right)+x_{1}^{2}\left(-48 x_{1}^{0^{2}} x_{2}^{0^{4}}+16 x_{2}^{4} x_{1}^{0^{2}}-28 x_{2}^{2} x_{1}^{0^{2}} x_{2}^{0^{2}}\right) \\
& +x_{1}\left(-76 x_{1}^{0^{3}} x_{2}^{0^{4}}+8 \frac{x_{2}^{5} x_{1}^{0^{3}}}{x_{2}^{0}}+4 x_{2}^{4} x_{1}^{0^{3}}-40 x_{2}^{3} x_{1}^{0^{3}} x_{2}^{0}-56 x_{2}^{2} x_{1}^{0^{3}} x_{2}^{0^{2}}\right. \\
& \left.\left.\left.-32 x_{2} x_{1}^{0^{3}} x_{2}^{0^{3}}\right)-8 x_{1}^{0^{4}} x_{2}^{0^{4}}\right]+1 \leftrightarrow 2\right\} \\
& D_{q g}^{G,(1)}\left(x_{1}^{0}, x_{2}^{0}\right)=\frac{\pi}{8 N} T_{f}\left\{\int d x_{2} H_{q g}\left(x_{1}^{0}, x_{2}, \mu_{F}^{2}\right)\right)\left[4 \frac{x_{2}^{0}}{x_{2}^{2}}-4 \frac{x_{2}^{0^{2}}}{x_{2}^{3}}+\left(\frac{2}{x_{2}}-4 \frac{x_{2}^{0}}{x_{2}^{2}}+4 \frac{x_{2}^{0^{2}}}{x_{2}^{3}}\right) \mathcal{K}_{a_{2}}\right. \\
& +\int d x_{1} \int d x_{2} \frac{H_{q g, 1}\left(x_{1}, x_{2}, \mu_{F}^{2}\right)}{\left(x_{1}-x_{1}^{0}\right)}\left[\frac{2}{x_{2}}-4 \frac{x_{2}^{0}}{x_{2}^{2}}+4 \frac{x_{2}^{0^{2}}}{x_{2}^{3}}\right] \\
& +\int d x_{1} H_{q g}\left(x_{1}, x_{2}^{0}, \mu_{F}^{2}\right)\left[8 \frac{x_{1}^{0}}{x_{1}^{2}}+\left(8 \frac{x_{1}^{0}}{x_{1}^{2}}-\frac{16}{x_{1}}+\frac{16}{x_{1}^{0}}\right) \mathcal{K}_{a_{1}}\right] \\
& +\int d x_{1} \int d x_{2} \frac{H_{q g, 2}\left(x_{1}, x_{2}, \mu_{F}^{2}\right)}{\left(x_{2}-x_{2}^{0}\right)}\left[8 \frac{x_{1}^{0}}{x_{1}^{2}}-\frac{16}{x_{1}}+\frac{16}{x_{1}^{0}}\right] \\
& +\int d x_{1} \int d x_{2} \frac{H_{q g}\left(x_{1}, x_{2}, \mu_{F}^{2}\right)}{\left(x_{1}+x_{1}^{0}\right)\left(x_{2}+x_{2}^{0}\right)\left(x_{1} x_{2}^{0}+x_{1}^{0} x_{2}\right)^{3}}\left[\frac { 1 } { x _ { 1 } ^ { 2 } } \left(-4 x_{1}^{0^{5}} x_{2}^{0^{3}}\right.\right.
\end{aligned}
$$




$$
\begin{aligned}
& \left.-8 x_{2}^{3} x_{1}^{0^{5}}-16 x_{2}^{2} x_{1}^{0^{5}} x_{2}^{0}-16 x_{2} x_{1}^{0^{5}} x_{2}^{0^{2}}-4 \frac{x_{1}^{0^{5}} x_{2}^{0^{4}}}{x_{2}}\right)+\frac{1}{x_{1}}\left(-32 x_{1}^{0^{4}} x_{2}^{0^{3}}\right. \\
& \left.+8 x_{2}^{3} x_{1}^{0^{4}}-8 x_{2}^{2} x_{1}^{0^{4}} x_{2}^{0}-44 x_{2} x_{1}^{0^{4}} x_{2}^{0^{2}}-12 \frac{x_{1}^{0^{4}} x_{2}^{0^{4}}}{x_{2}}-8 \frac{x_{1}^{0^{4}} x_{2}^{0^{5}}}{x_{2}^{2}}\right) \\
& +x_{1}^{4}\left(16 \frac{x_{2}^{0^{3}}}{x_{1}^{0}}+32 \frac{x_{2} x_{2}^{0^{2}}}{x_{1}^{0}}\right)+x_{1}^{3}\left(-2 x_{2}^{0^{3}}+32 x_{2}^{2} x_{2}^{0}+16 x_{2} x_{2}^{0^{2}}+2 \frac{x_{2}^{0^{4}}}{x_{2}}\right. \\
& \left.-4 \frac{x_{2}^{0^{6}}}{x_{2}^{3}}\right)+x_{1}^{2}\left(-30 x_{1}^{0} x_{2}^{0^{3}}+16 x_{2}^{3} x_{1}^{0}-78 x_{2} x_{1}^{0} x_{2}^{0^{2}}+4 \frac{x_{1}^{0} x_{2}^{0^{4}}}{x_{2}}-12 \frac{x_{1}^{0} x_{2}^{0^{5}}}{x_{2}^{2}}\right. \\
& \left.-8 \frac{x_{1}^{0} x_{2}^{0^{6}}}{x_{2}^{3}}\right)+x_{1}\left(-32 x_{1}^{0^{2}} x_{2}^{0^{3}}-30 x_{2}^{2} x_{1}^{0^{2}} x_{2}^{0}-30 x_{2} x_{1}^{0^{2}} x_{2}^{0^{2}}-32 \frac{x_{1}^{0^{2}} x_{2}^{0^{4}}}{x_{2}}\right. \\
& \left.-24 \frac{x_{1}^{0^{2}} x_{2}^{0^{5}}}{x_{2}^{2}}-8 \frac{x_{1}^{0^{2}} x_{2}^{0^{6}}}{x_{2}^{3}}\right)-52 x_{1}^{0^{3}} x_{2}^{0^{3}}+2 x_{2}^{3} x_{1}^{0^{3}}+10 x_{2}^{2} x_{1}^{0^{3}} x_{2}^{0}-36 \frac{x_{1}^{0^{3}} x_{2}^{0^{4}}}{x_{2}} \\
& \left.-16 \frac{x_{1}^{0^{3}} x_{2}^{0^{5}}}{x_{2}^{2}}-8 \frac{x_{1}^{0^{3}} x_{2}^{0^{6}}}{x_{2}^{3}}\right), \\
D_{g q}^{G,(1)}\left(x_{1}^{0}, x_{2}^{0}\right)= & \left.D_{q g}^{G,(1)}\left(x_{1}^{0}, x_{2}^{0}\right)\right|_{1 \leftrightarrow 2} \cdot(\mathrm{B} .8)
\end{aligned}
$$




\section{References}

[1] N. Arkani-Hamed, S. Dimopoulos, and G. Dvali, Phys. Lett B429, (1998) 263; I. Antoniadis, N. Arkani-Hamed, S. Dimopoulos, and G. Dvali, Phys. Lett. B436, (1998) 257; N. Arkani-Hamed, S. Dimopoulos, and G. Dvali, Phys. Rev. D59, (1999) 086004.

[2] G. Giudice, R. Rattazzi, and J. Wells, Nucl. Phys. B544, (1999) 3 and revised version 2, hep-ph/9811291.

[3] T. Han, J.D. Lykken, and R.-J. Zhang, Phys. Rev. D 59, (1999) 105006 and revised version 4 , hep-ph/9811350.

[4] For an exhaustive list of references on the subject, see: A. Perez-Lorenzana, AIP Conf.Proc.562 (2001) 53, hep-ph/0008333; K. Sridhar, Int. J. Mod. Phys. A 15 (2000) 2397, hep-ph/0004053; K. Cheung, hep-ph/0305003.

[5] P. Mathews, V. Ravindran, K. Sridhar, J. High Energy Phys. 08 (2004) 048, hep-ph/0405292.

[6] G. Altarelli, R.K. Ellis and G. Martinelli, Nucl. Phys. B157 (1979) 461.

[7] B. Humpert and W.L. van Neerven, Phys. Lett. B84 (1979) 327, Erratum: B85 (1979) 471; ibid. B89 (1979) 69; ibid. Nucl. Phys. B184 (1981) 225.

[8] J.L. Hewett, Phys. Rev. Lett. 82 (1999) 4765, hep-ph/9811356;

[9] A. K. Gupta, N. K. Mondal and S. Raychaudhuri, hep-ph/9904234; K. Cheung and G. Landsberg, Phys. Rev. D62, (2000) 076003; K. Cheung, Phys. Lett. B460, (1999) 383.

[10] L. Randall and R. Sundrum, Phys. Rev. Lett. 83 (1999) 3370.

[11] J. Kubar-André and F.E. Paige, Phys. Rev. D19 (1979) 221.

[12] G. Altarelli and G. Parisi, Nucl. Phys. B126 (1977) 298.

[13] J. Kubar, M. le Bellac, J.L. Meunier and G. Plaut, Nucl. Phys. B175 (1980) 251.

[14] P. Aurenche and P. Chiappetta, Z. Phys. C34 (1987) 201.

[15] P.J. Sutton, A.D. Martin, R.G. Roberts, W.J. Stirling, Phys. Rev. D45 (1992) 2349 .

[16] P.J. Rijken and W.L. van Neerven, Phys. Rev. D51 (1995) 44. 
[17] A. D. Martin, R. G. Roberts, W. J. Stirling and R. S. Thorne, Eur. Phys. J. C23 (2002) 73, hep-ph/0110215.

[18] M. Sanders, To appear in the proceedings of the International Europhysics Conference on High-Energy Physics, Aachen, (2003) eprint hep-ex/0310033; B. Abbott et al., Phys. Rev. Lett. 86 (2001) 1151.

[19] U. Baur, S. Keller and W. K. Sakumoto, Phys. Rev. D57 (1998) 199; B. Kamal, Phys. Rev. D57 (1998) 6663. 


\section{Figure Captions}

Fig. 10a The invariant di-lepton pair mass distribution $d \sigma / d Q$ for $M_{s}=2 \mathrm{TeV}$ and $d=3$ at $\sqrt{S}=14 \mathrm{TeV}$ (LHC). Standard model (dotted line), gravity (long dashed line), standard model plus gravity (solid line).

Fig. 10b The double differential cross section $d^{2} \sigma / d Q d x_{F}$ where $x_{F}$ is the longitudinal momentum fraction of the di-lepton pair. The plot is given for $M_{s}=2 \mathrm{TeV}$ and $d=3$ at $\sqrt{S}=14 \mathrm{TeV}$ and $Q=700 \mathrm{GeV}$ (LHC). Standard model (dotted line), gravity (long dashed line), standard model plus gravity (solid line).

Fig. 10c The double differential cross section $d^{2} \sigma / d Q d Y$ where $Y$ is the rapidity of the di-lepton pair. The plot is given for $M_{s}=2 \mathrm{TeV}$ and $d=3$ at $\sqrt{S}=14 \mathrm{TeV}$ and $Q=700 \mathrm{GeV}$ (LHC). Standard model (dotted line), gravity (long dashed line), standard model plus gravity (solid line).

Fig. 11a The $K^{I}$-factor for the cross section $d \sigma / d Q$ at $M_{s}=2 \mathrm{TeV}$ and $d=3$. The plot is made for the LHC $(\sqrt{S}=14 \mathrm{TeV})$. Standard model (dotted line), gravity (long dashed line), standard model plus gravity (solid line).

Fig. 11b The $K^{I}$-factor for the double differential cross section $d^{2} \sigma / d Q d x_{F}$ at $M_{s}=$ $2 \mathrm{TeV}$ and $d=3$. The plot is made for the LHC with $\sqrt{S}=14 \mathrm{TeV}$ and $Q=700 \mathrm{GeV}$. Standard model (dotted line), gravity (long dashed line), standard model plus gravity (solid line).

Fig. 11c The $K^{I}$-factor for the double differential cross section $d^{2} \sigma / d Q d Y$ at $M_{s}=$ $2 \mathrm{TeV}$ and $d=3$. The plot is made for the LHC with $\sqrt{S}=14 \mathrm{TeV}$ and $Q=700 \mathrm{GeV}$. Standard model (dotted line), gravity (long dashed line), standard model plus gravity (solid line).

Fig. 12a The ratio $R^{I}$ Eq. (7.7) for the cross section $d \sigma / d Q$ at $M_{s}=2 \mathrm{TeV}$ and $d=3$. The plot is made for the LHC $(\sqrt{S}=14 \mathrm{TeV})$. Gravity alone in LO (dot-dashed line), standard model plus gravity in LO (short dashed line), Gravity alone in NLO (long dashed line), standard model plus gravity in NLO (solid line),

Fig. 12b The ratio $R^{I}$ Eq. (7.8) for the cross section $d^{2} \sigma / d Q d x_{F}$ at $M_{s}=2 \mathrm{TeV}$ and $d=3$. The plot is made for the LHC with $\sqrt{S}=14 \mathrm{TeV}), Q=700 \mathrm{GeV}$ and $x_{F}=0$. Gravity alone in LO (dot-dashed line), standard model plus gravity in LO (short dashed line), Gravity alone in NLO (long dashed line), standard model plus gravity in NLO (solid line),

Fig. 12c The ratio $R^{I}$ Eq. (7.9) for the cross section $d^{2} \sigma / d Q d Y$ at $M_{s}=2 \mathrm{TeV}$ and $d=3$. The plot is made for the LHC with $\sqrt{S}=14 \mathrm{TeV}$ ), $Q=700 \mathrm{GeV}$ and $Y=0$. Gravity alone in LO (dot-dashed line), standard model plus gravity 
in LO (short dashed line), Gravity alone in NLO (long dashed line), standard model plus gravity in NLO (solid line),

Fig. 13a The invariant di-lepton pair mass distribution $d \sigma / d Q$ for $M_{s}=2 \mathrm{TeV}$ and $d=2$ at $\sqrt{S}=1.96 \mathrm{TeV}$ (TEVATRON). Standard model (dotted line), gravity (long dashed line), standard model plus gravity (solid line).

Fig. 13b The double differential cross section $d^{2} \sigma / d Q d x_{F}$ where $x_{F}$ is the longitudinal momentum fraction of the di-lepton pair. The plot is given for $M_{s}=2 \mathrm{TeV}$ and $d=2$ at $\sqrt{S}=1.96 \mathrm{TeV}$ and $Q=400 \mathrm{GeV}$ (TEVATRON). Standard model (dotted line), gravity (long dashed line), standard model plus gravity (solid line).

Fig. 13c The double differential cross section $d^{2} \sigma / d Q d Y$ where $Y$ is the rapidity of the di-lepton pair. The plot is given for $M_{s}=2 \mathrm{TeV}$ and $d=2$ at $\sqrt{S}=1.96 \mathrm{TeV}$ and $Q=400 \mathrm{GeV}$ (TEVATRON). Standard model (dotted line), gravity (long dashed line), standard model plus gravity (solid line).

Fig. 14a The $K^{I}$-factor for the cross section $d \sigma / d Q$ at $M_{s}=2 \mathrm{TeV}$ and $d=2$. The plot is made for the TEVATRON $(\sqrt{S}=1.96 \mathrm{TeV})$. Standard model (dotted line), gravity (long dashed line), standard model plus gravity (solid line).

Fig. 14b The $K^{I}$-factor for the double differential cross section $d^{2} \sigma / d Q d x_{F}$ at $M_{s}=$ $2 \mathrm{TeV}$ and $d=2$. The plot is made for the TEVATRON with $\sqrt{S}=1.96 \mathrm{TeV}$ and $Q=400 \mathrm{GeV}$. Standard model (dotted line), gravity (long dashed line), standard model plus gravity (solid line).

Fig. 14c The $K^{I}$-factor for the double differential cross section $d^{2} \sigma / d Q d Y$ at $M_{s}=$ $2 \mathrm{TeV}$ and $d=2$. The plot is made for the TEVATRON with $\sqrt{S}=1.96 \mathrm{TeV}$ and $Q=400 \mathrm{GeV}$. Standard model (dotted line), gravity (long dashed line), standard model plus gravity (solid line).

Fig. 15a The ratio $R^{I}$ Eq. (7.7) for the cross section $d \sigma / d Q$ at $M_{s}=2 \mathrm{TeV}$ and $d=2$. The plot is made for the TEVATRON $(\sqrt{S}=1.96 \mathrm{TeV})$. Gravity alone in LO (dot-dashed line), standard model plus gravity in LO (short dashed line), Gravity alone in NLO (long dashed line), standard model plus gravity in NLO (solid line),

Fig. 15b The ratio $R^{I}$ Eq. (7.8) for the cross section $d^{2} \sigma / d Q d x_{F}$ at $M_{s}=2 \mathrm{TeV}$ and $d=2$. The plot is made for the TEVATRON with $\sqrt{S}=1.96 \mathrm{TeV}, Q=400 \mathrm{GeV}$ and $x_{F}=0$. Gravity alone in LO (dot-dashed line), standard model plus gravity in LO (short dashed line), Gravity alone in NLO (long dashed line), standard model plus gravity in NLO (solid line), 
Fig. 15c The ratio $R^{I}$ Eq. (7.9) for the cross section $d^{2} \sigma / d Q d Y$ at $M_{s}=2 \mathrm{TeV}$ and $d=2$. The plot is made for the TEVATRON with $\sqrt{S}=1.96 \mathrm{TeV}, Q=400 \mathrm{GeV}$ and $Y=0$. Gravity alone in LO (dot-dashed line), standard model plus gravity in LO (short dashed line), Gravity alone in NLO (long dashed line), standard model plus gravity in NLO (solid line),

Fig. 16 The forward backward asymmetry $A_{F B}$ in NLO for the TEVATRON $\sqrt{S}=$ $1.96 \mathrm{TeV}$. The parameters are $M_{s}=1.5 \mathrm{TeV}$ and $d=2$. Standard model (dotted line), standard model plus gravity (solid line). 


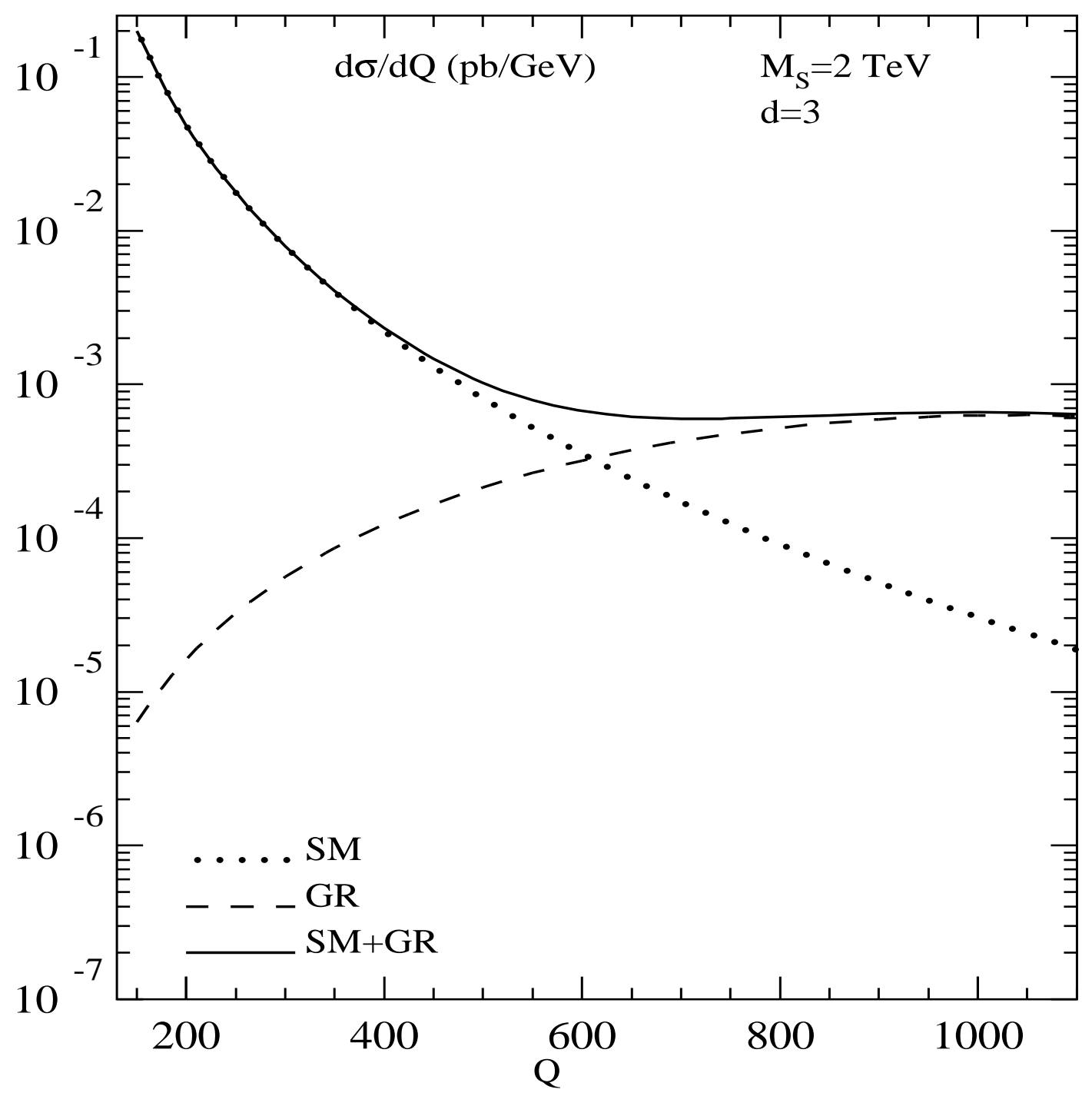

Fig. 10a 


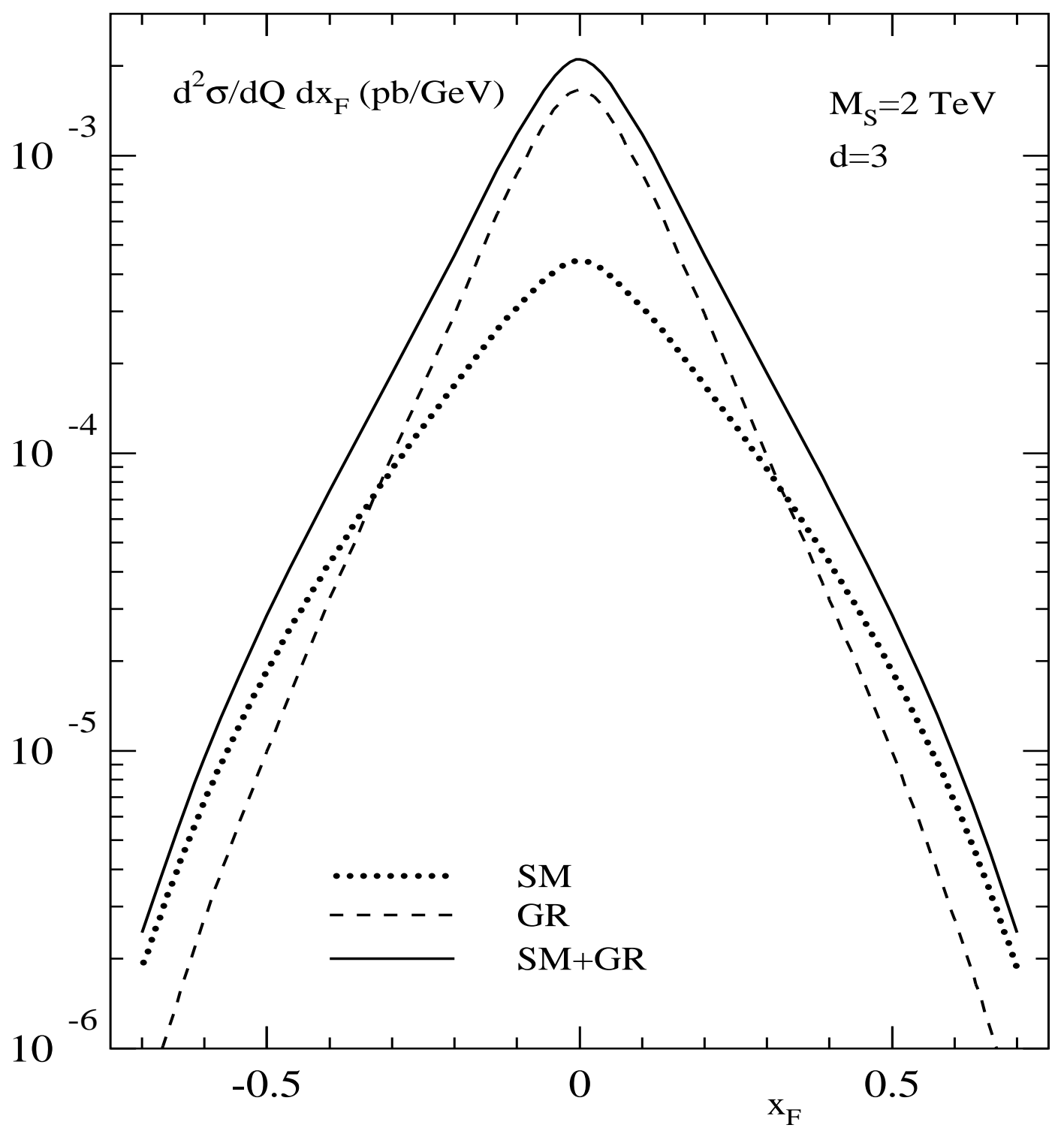

Fig. 10b 


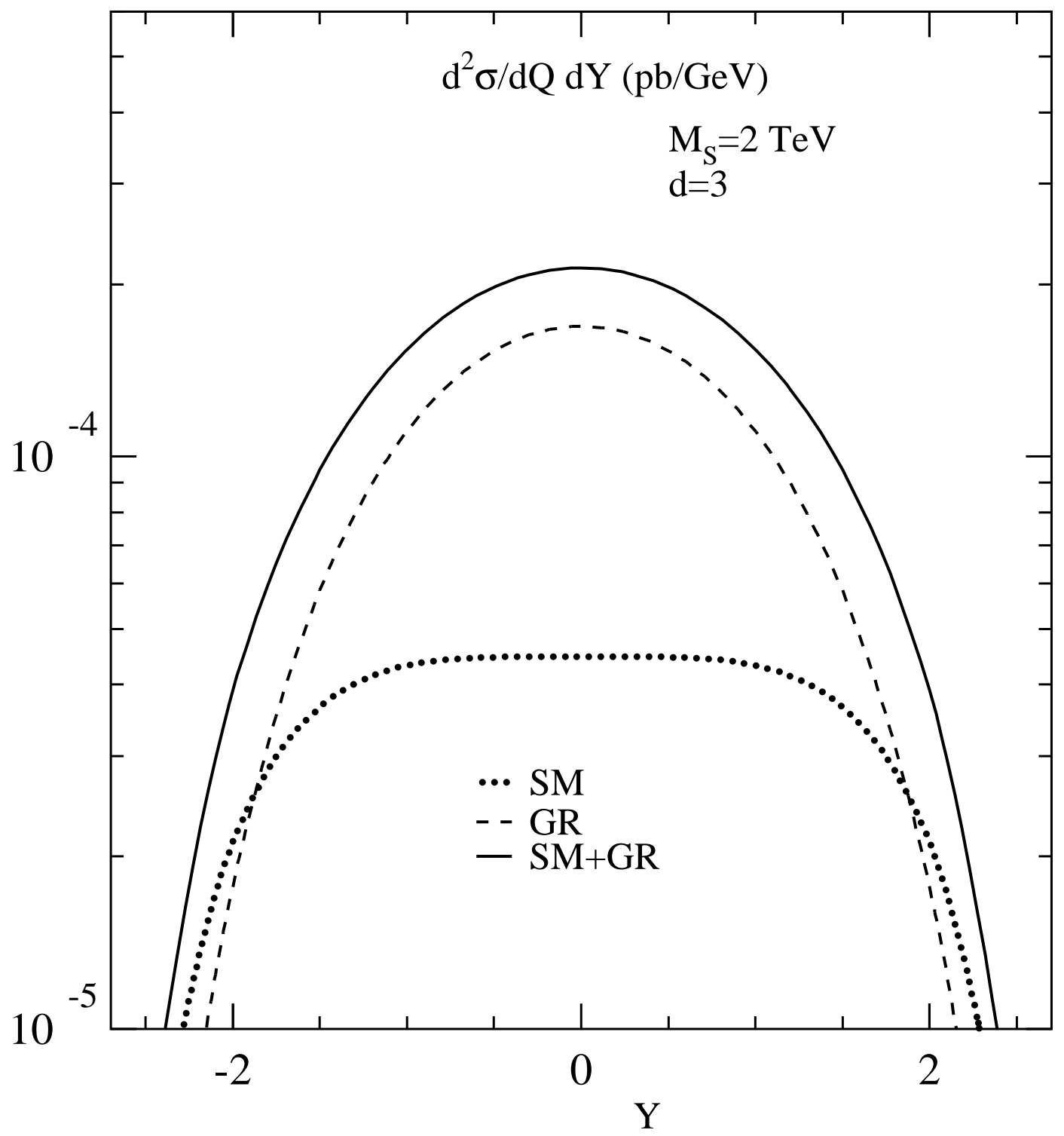

Fig. 10c 


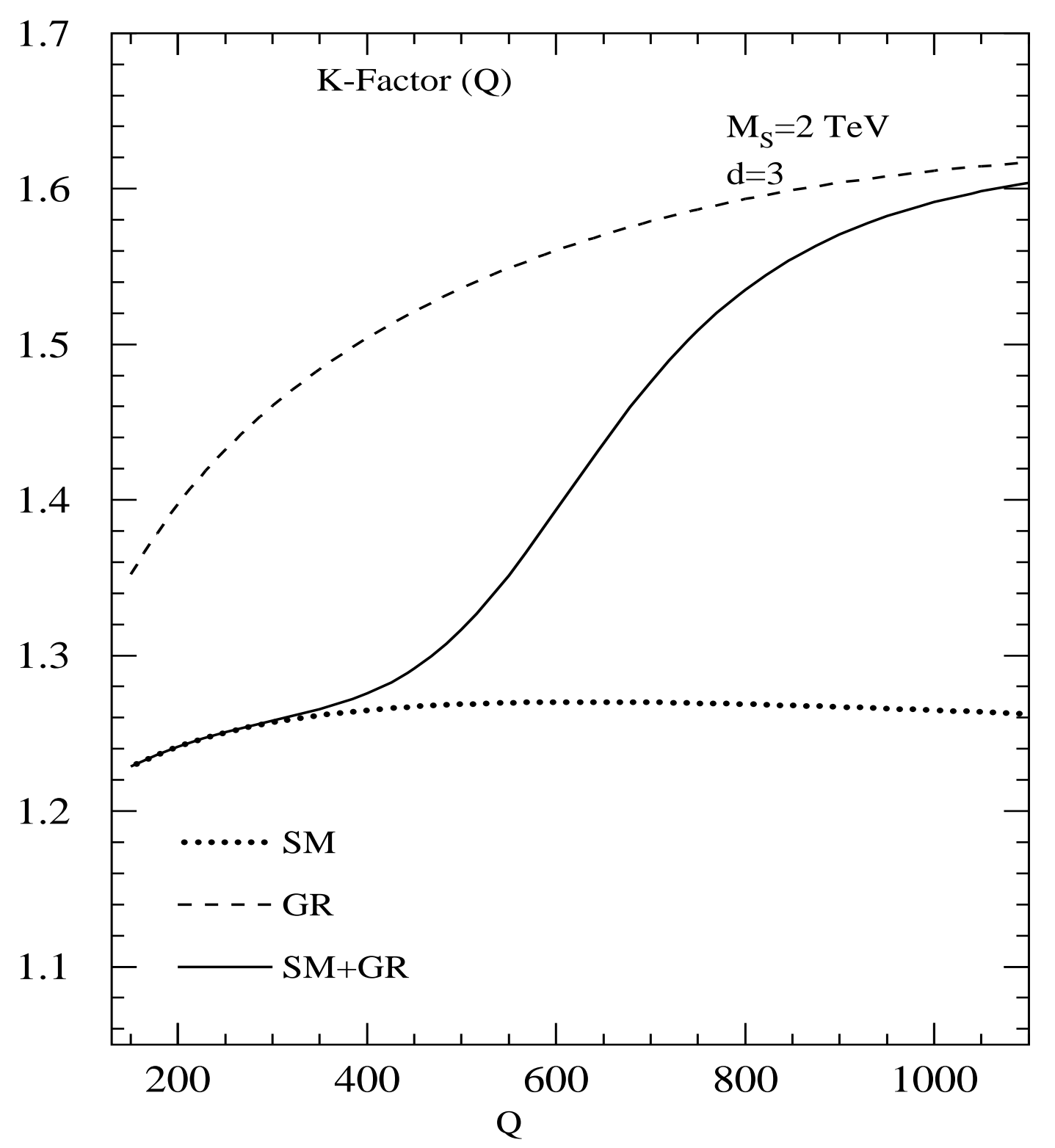

Fig. 11a 


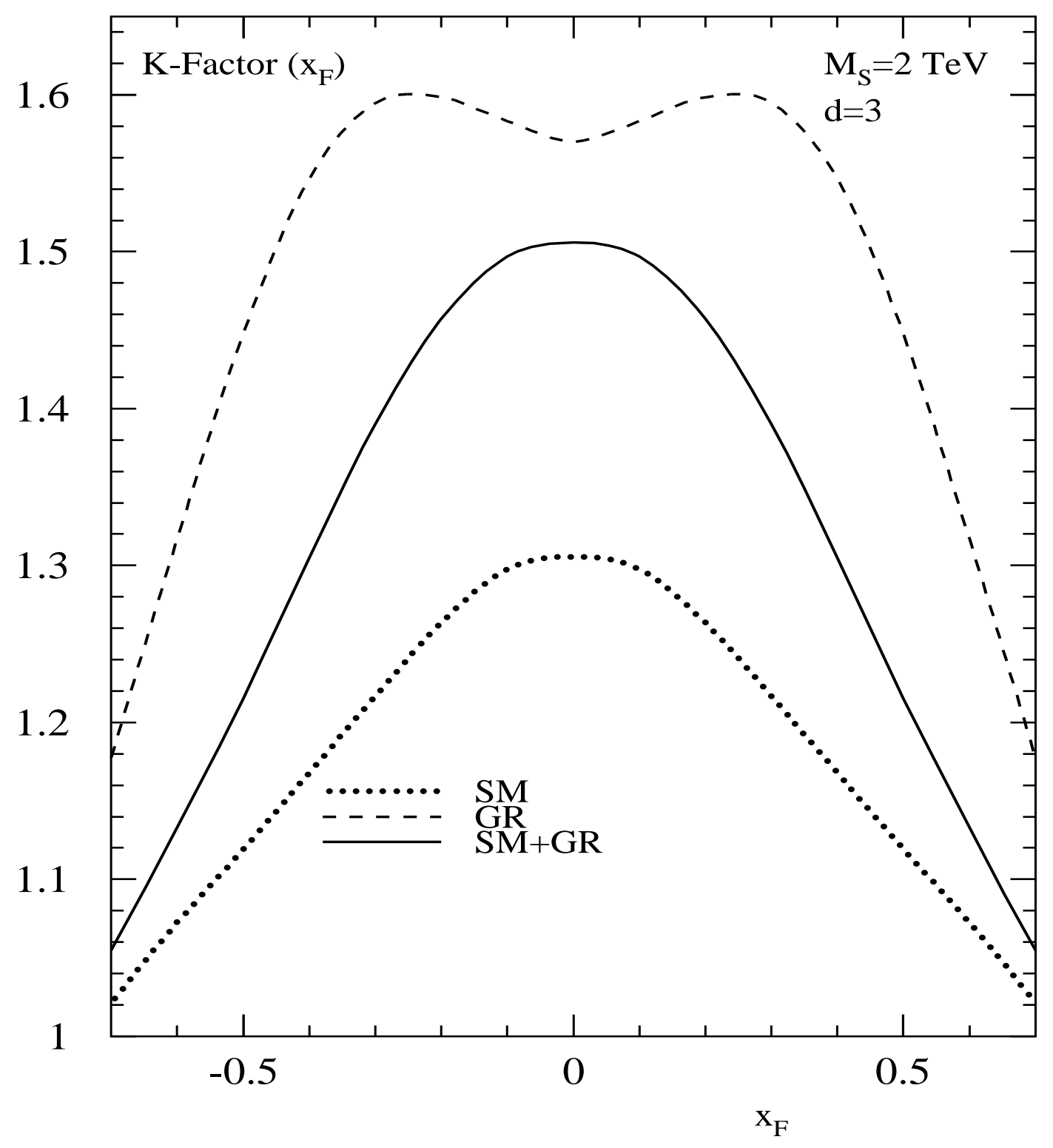

Fig. 11b 


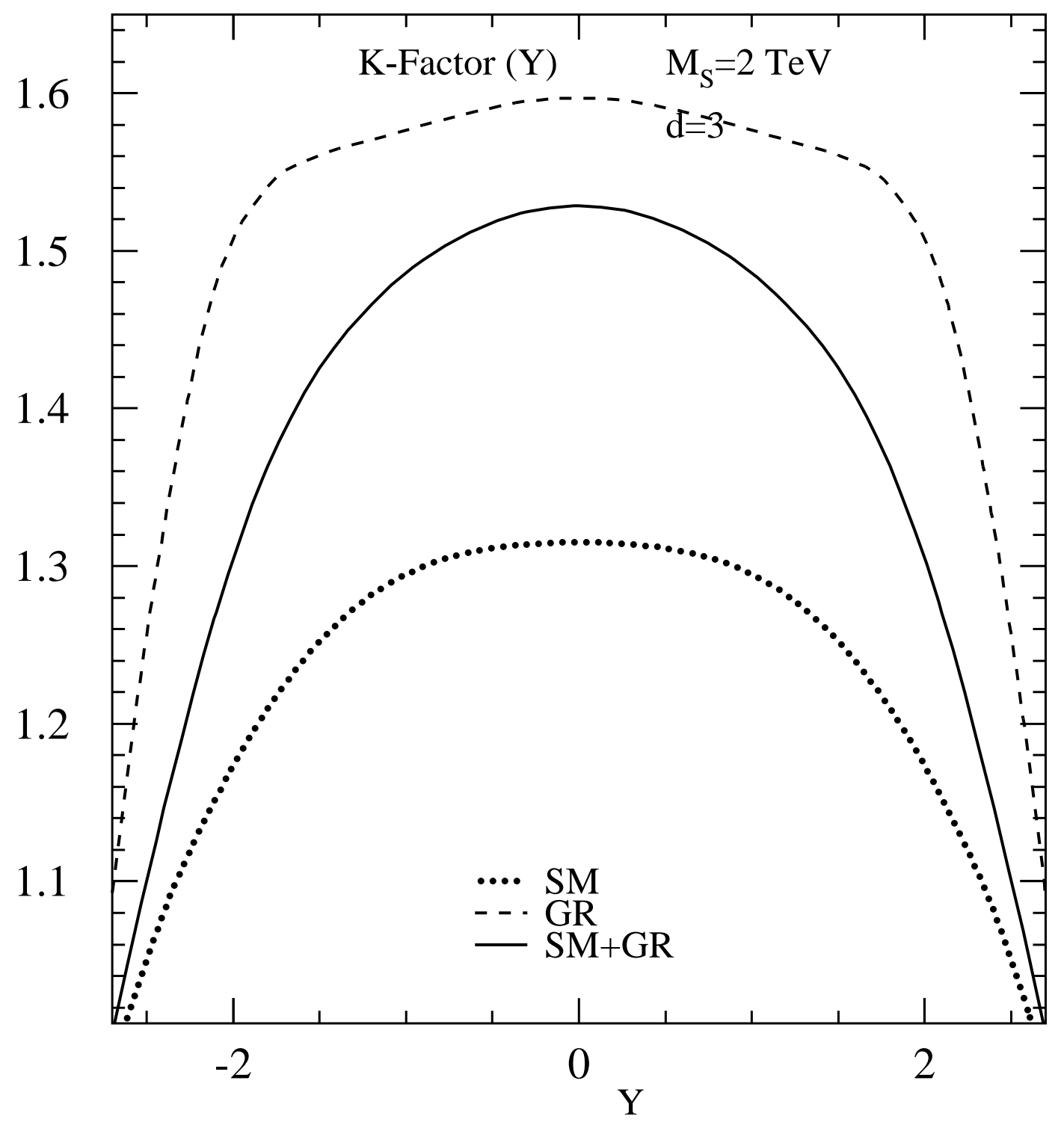

Fig. 11c 


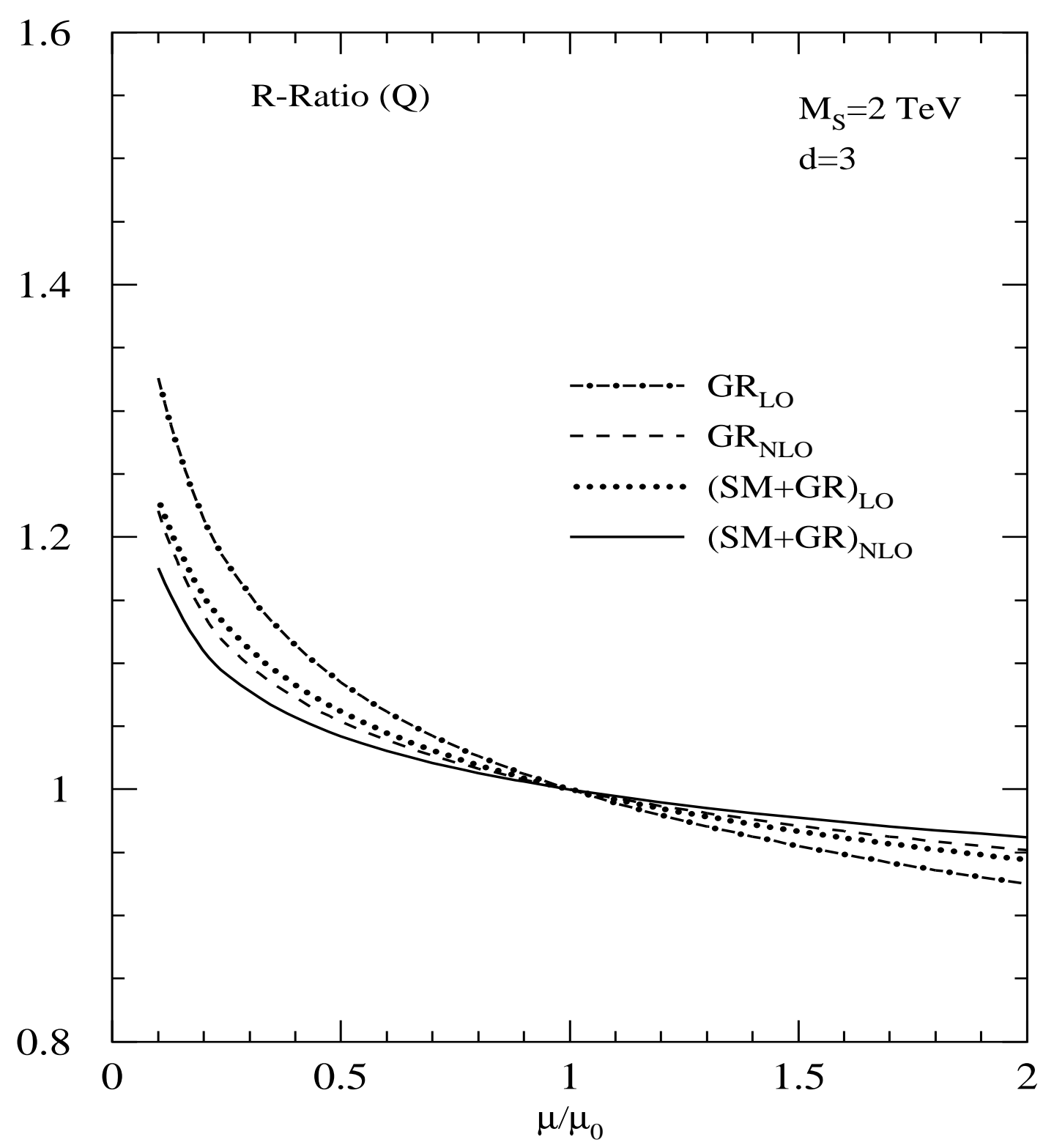

Fig. 12a 


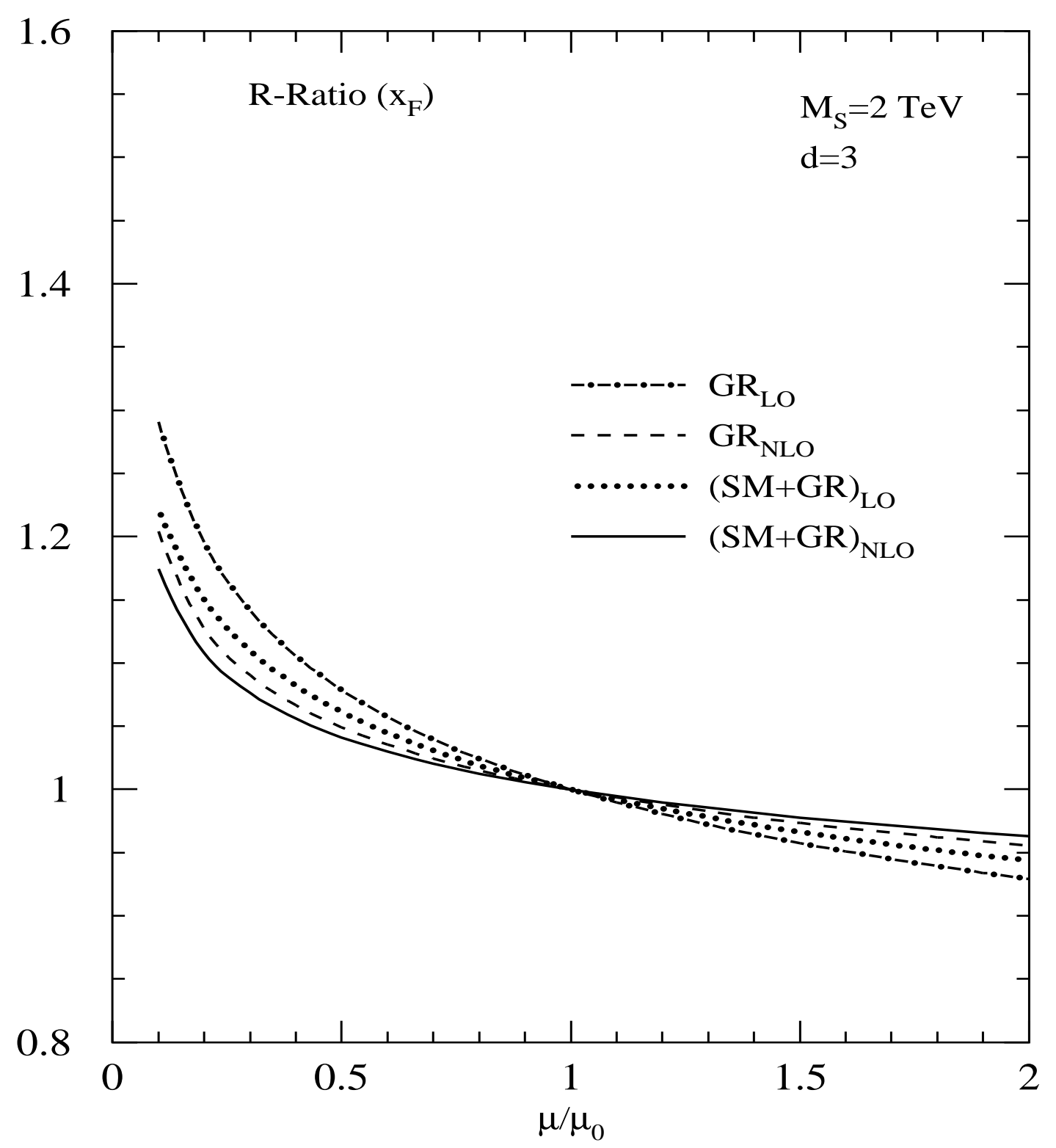

Fig. 12b 


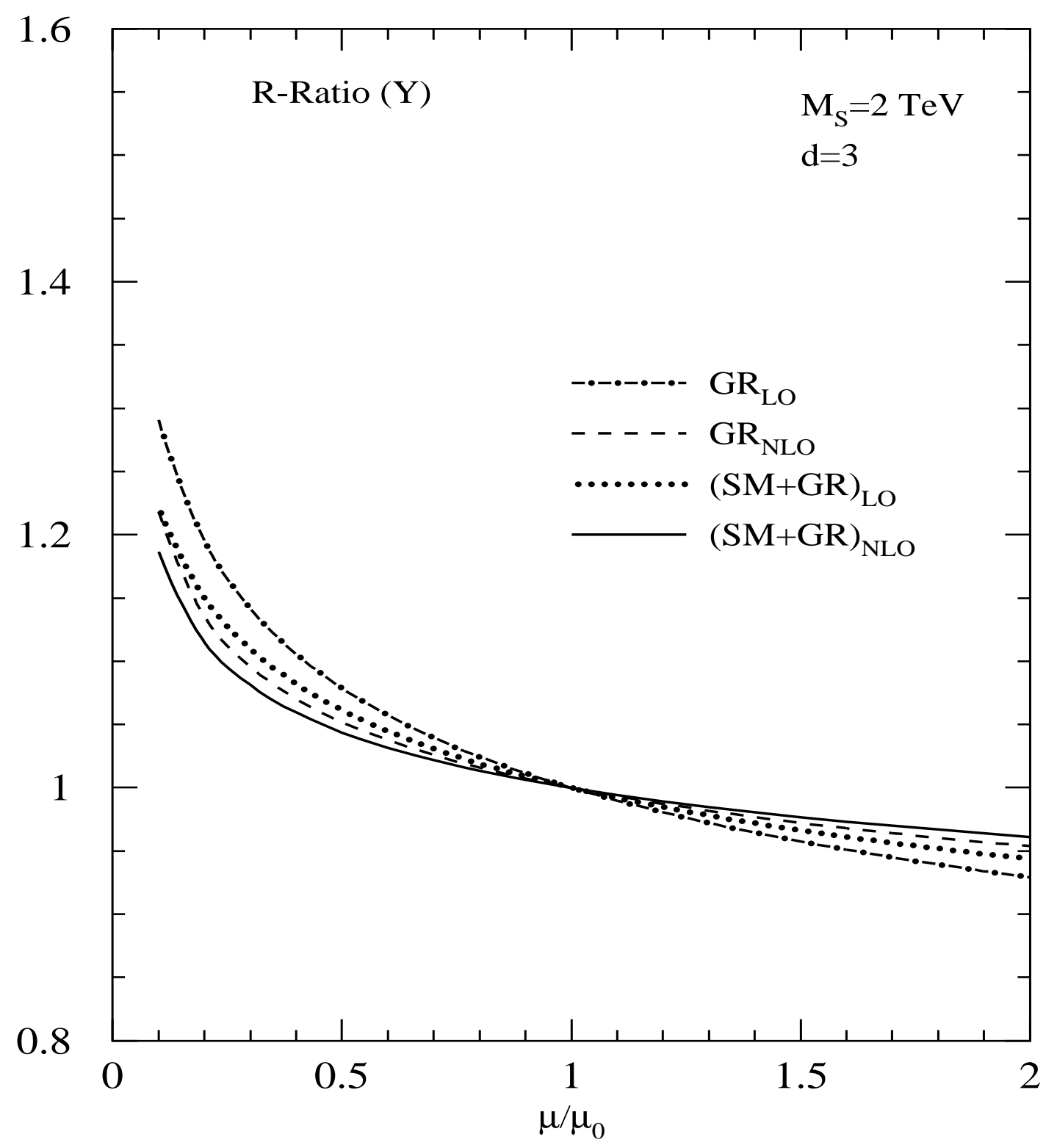

Fig. 12c 


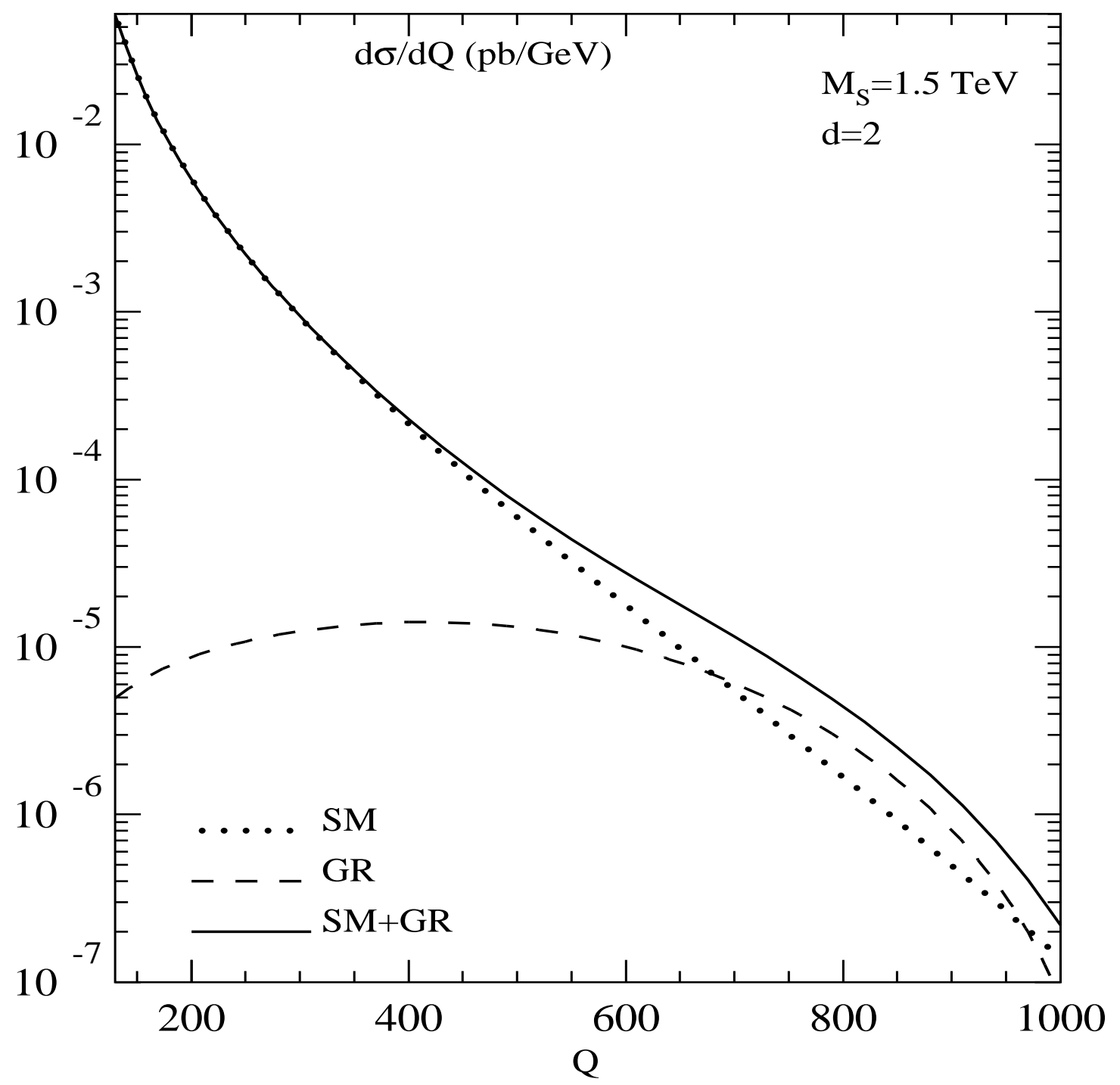

Fig. 13a 


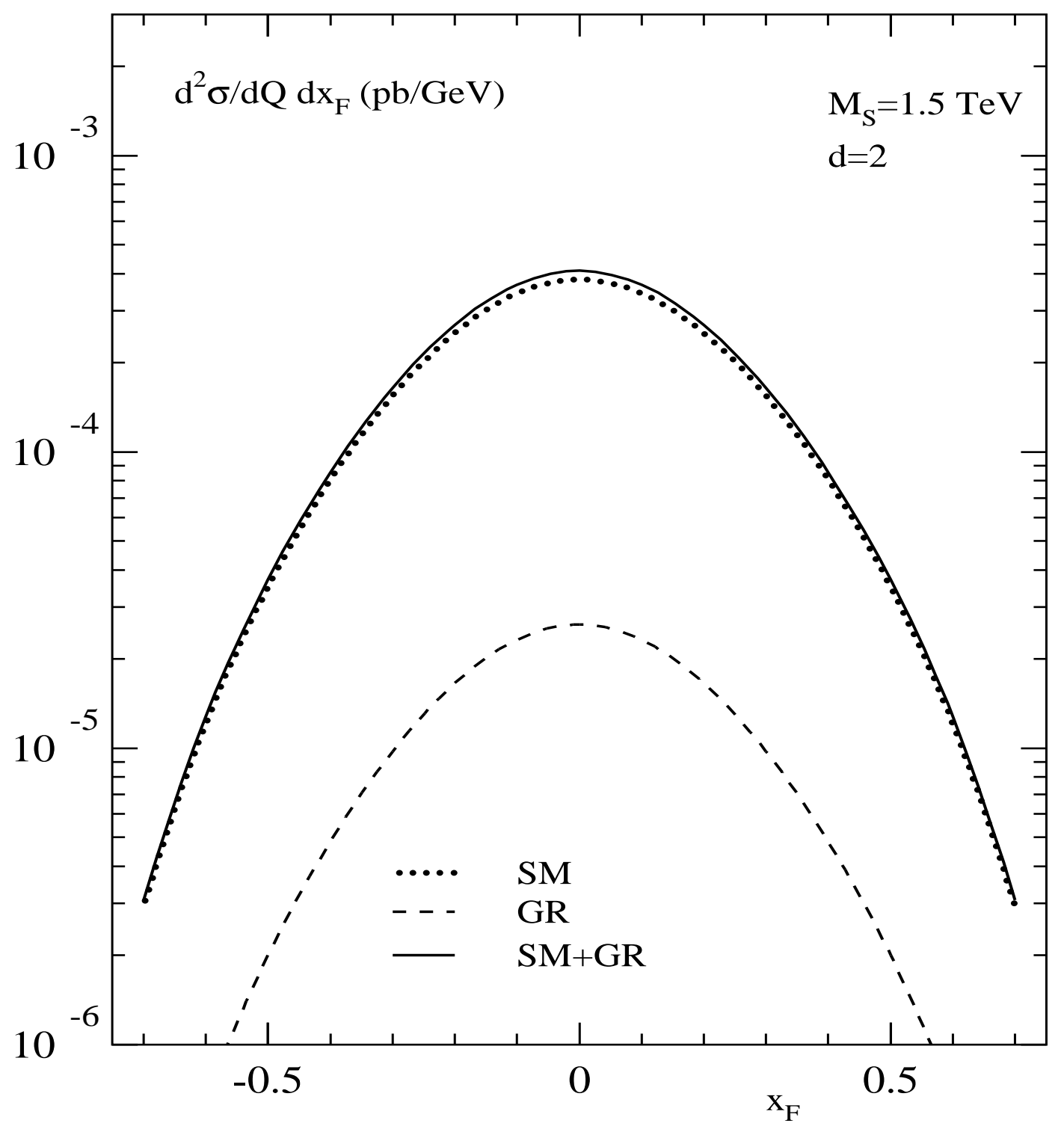

Fig. 13b 


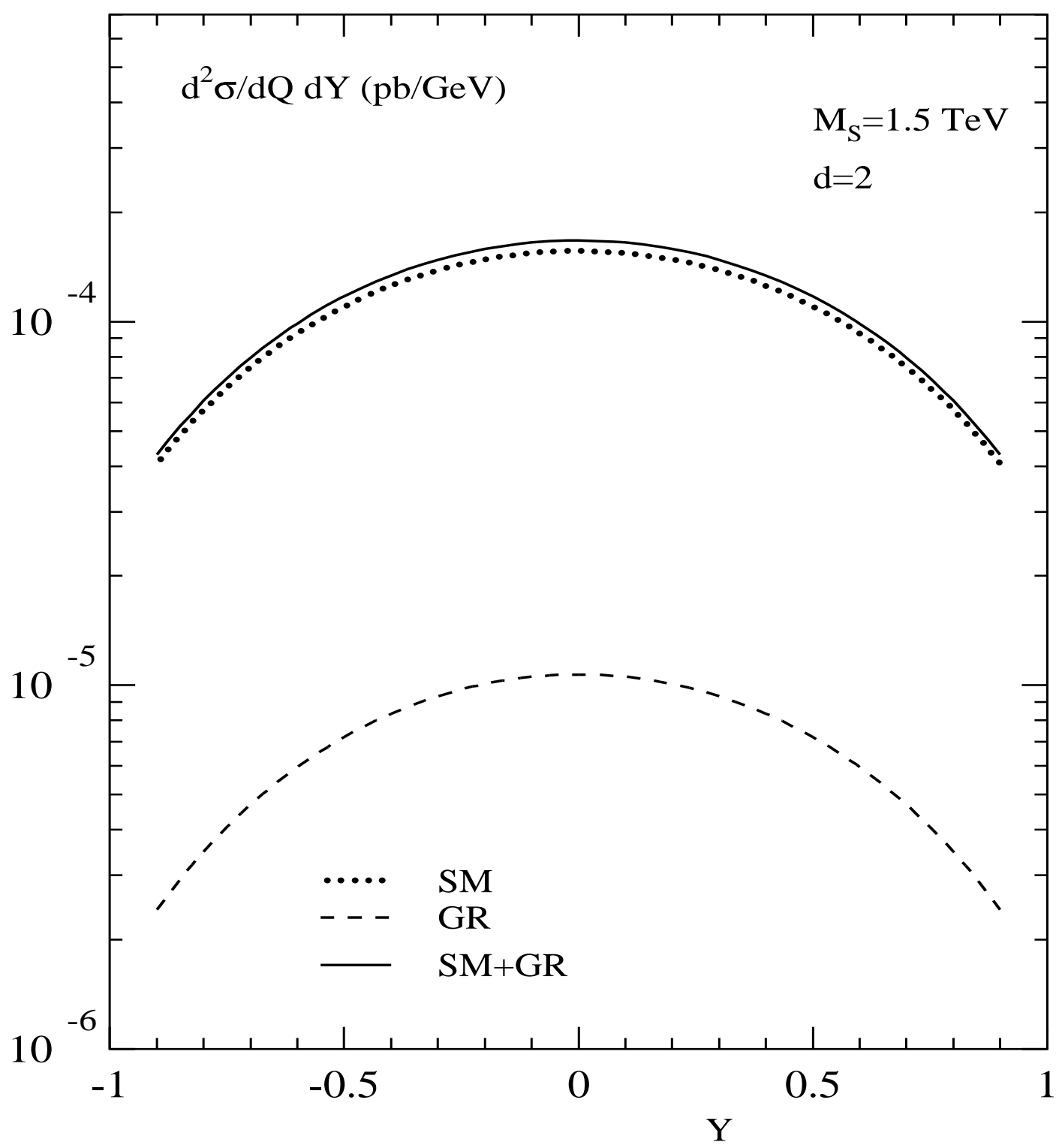

Fig. 13c 


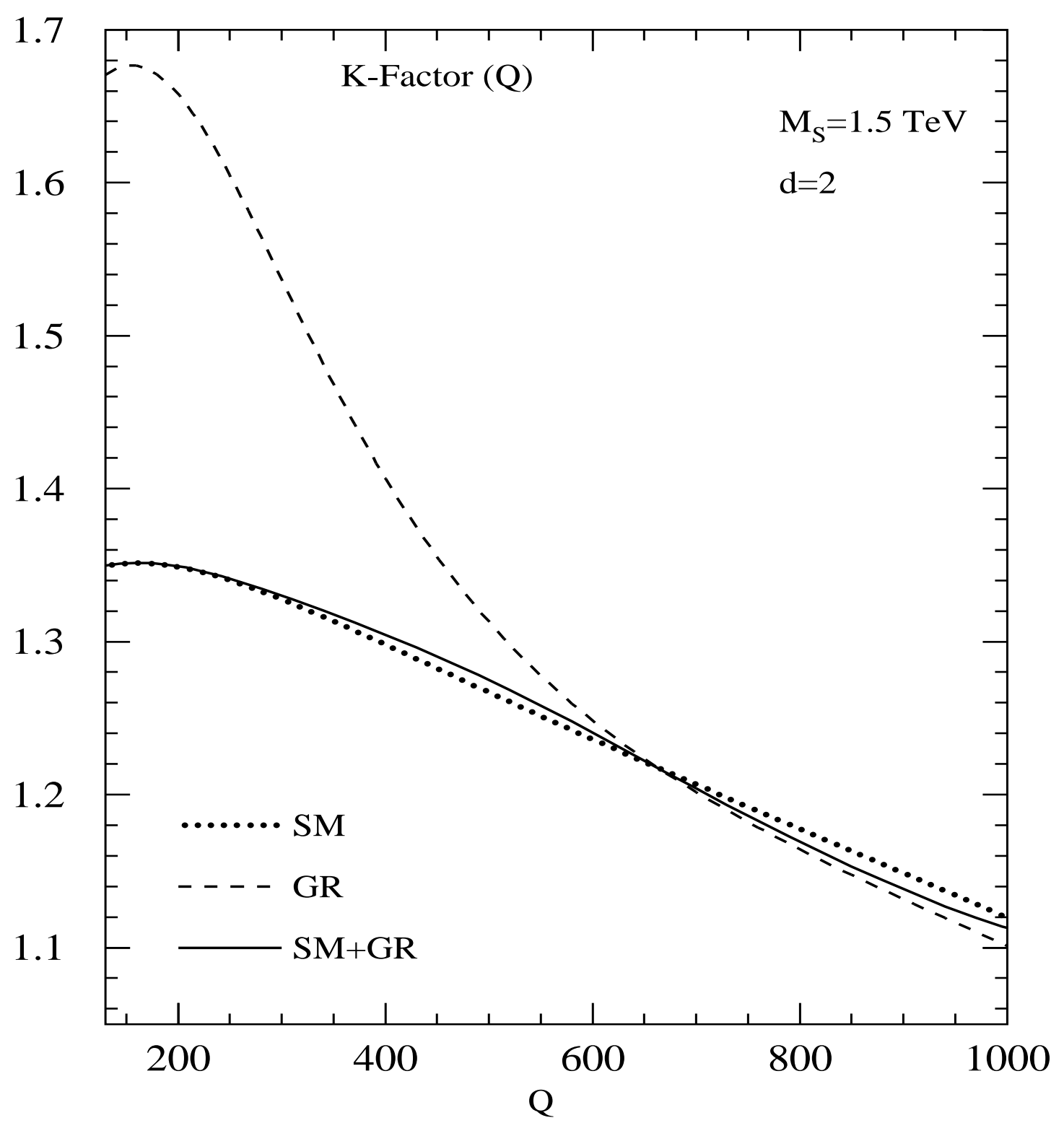

Fig. 14a 


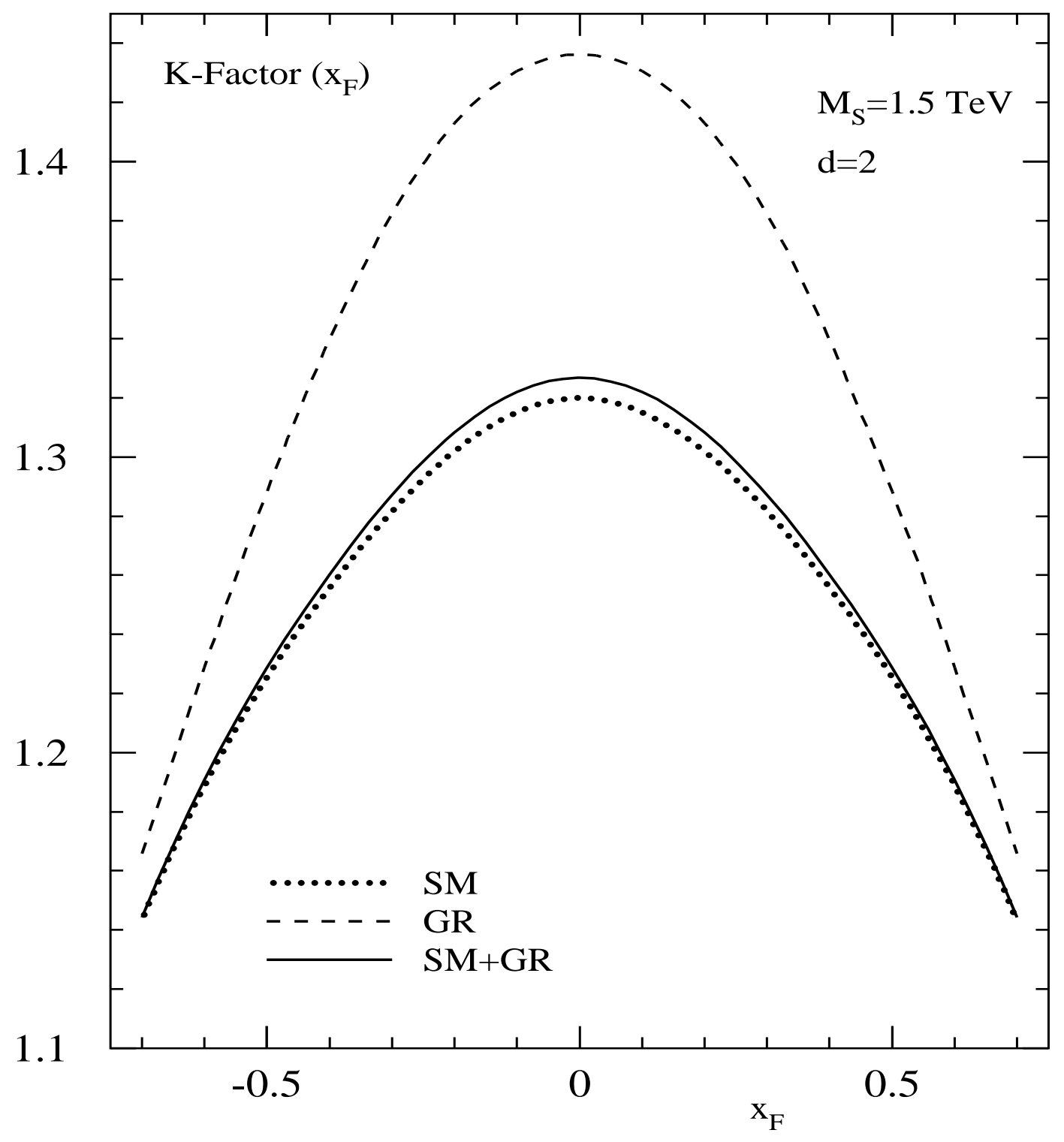

Fig. 14b 


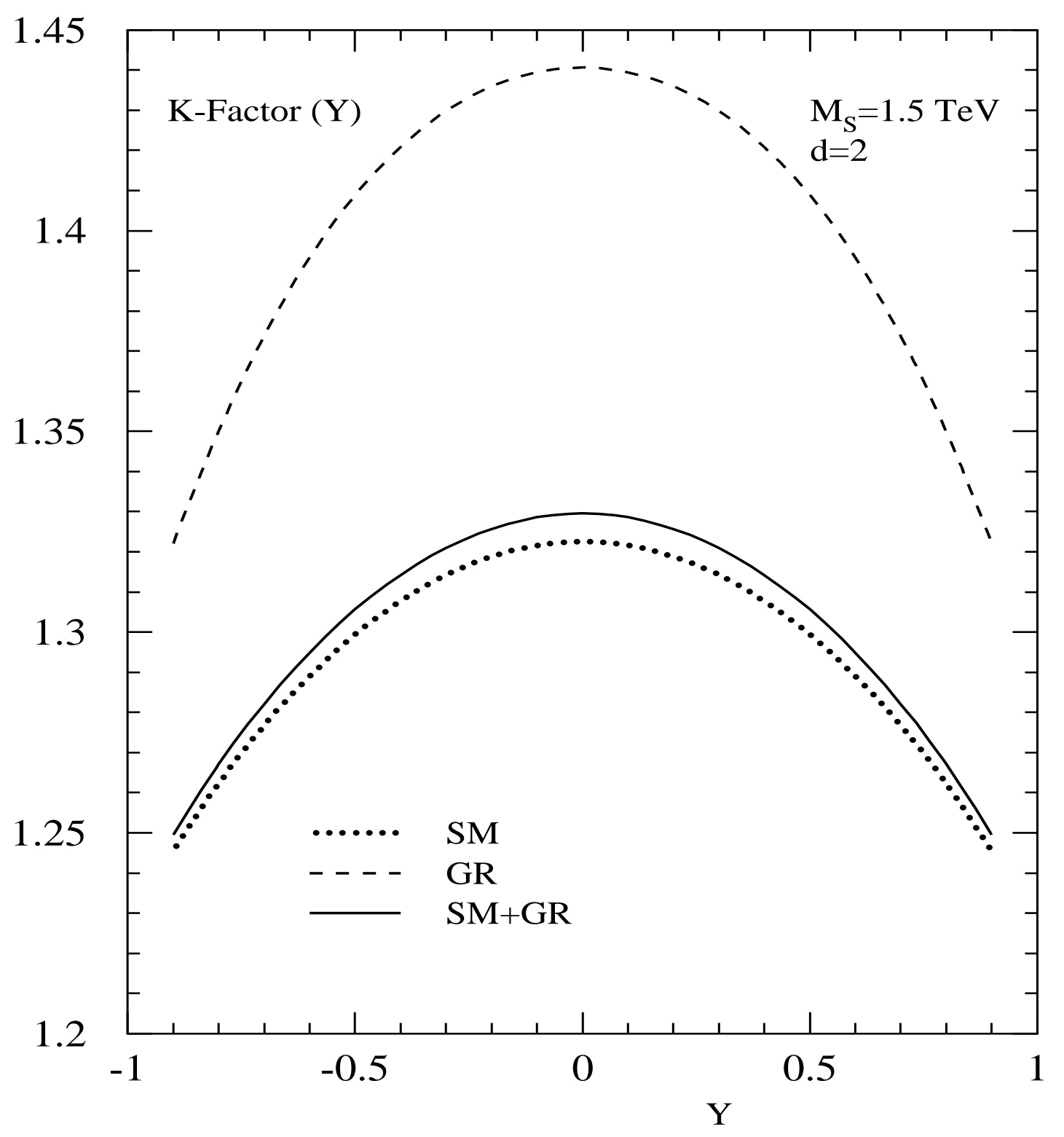

Fig. 14c 


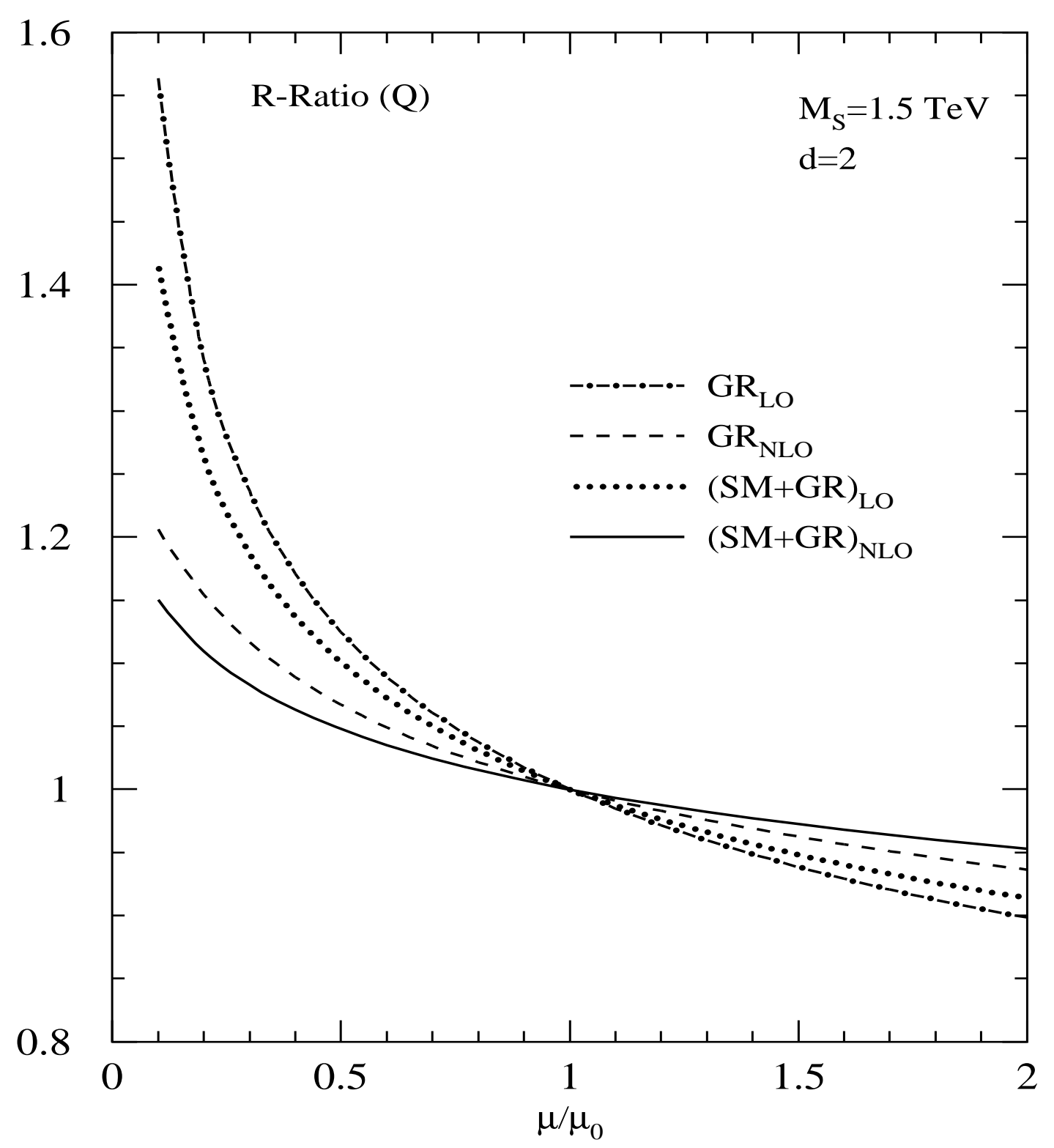

Fig. 15a 


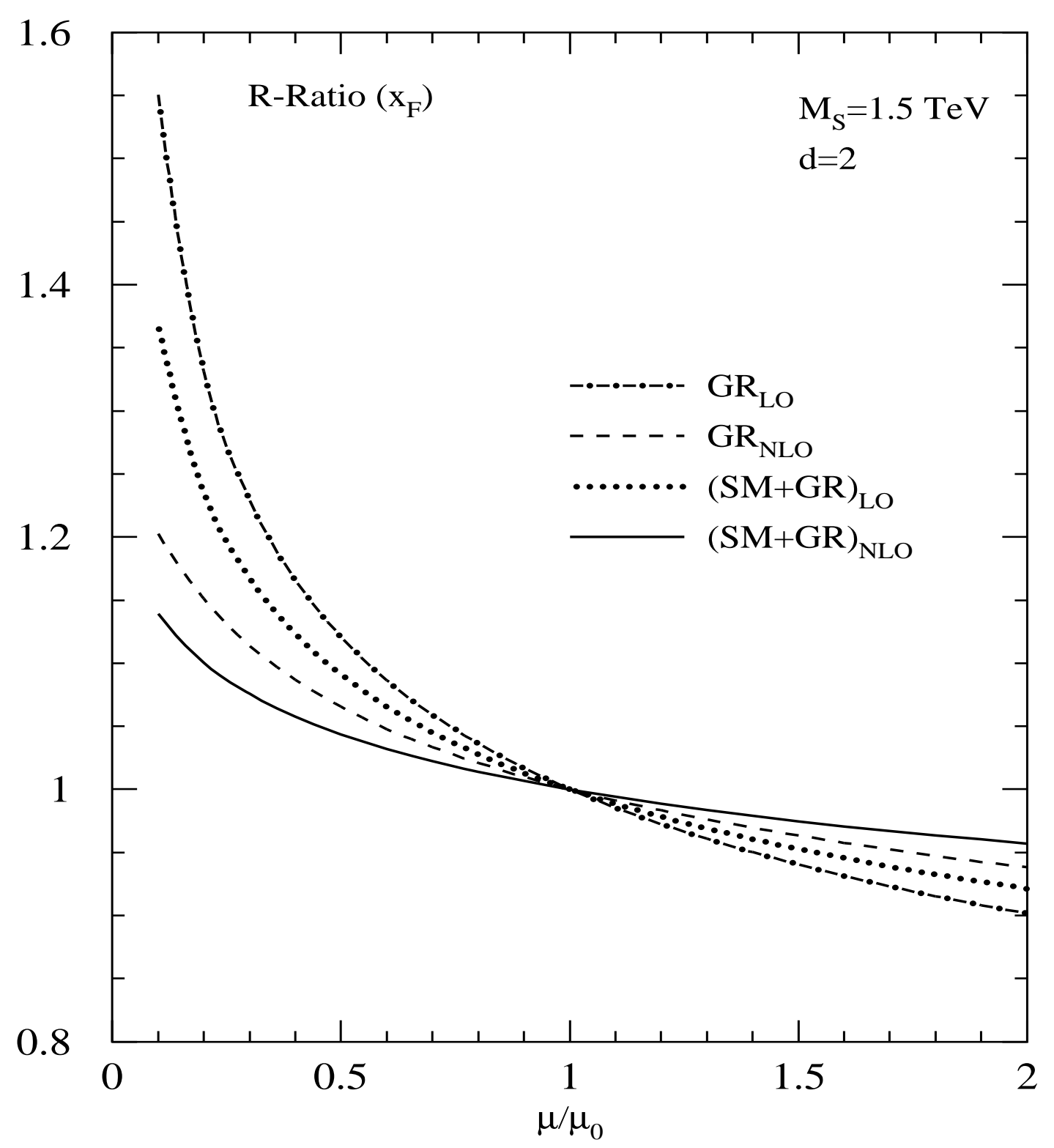

Fig. 15b 


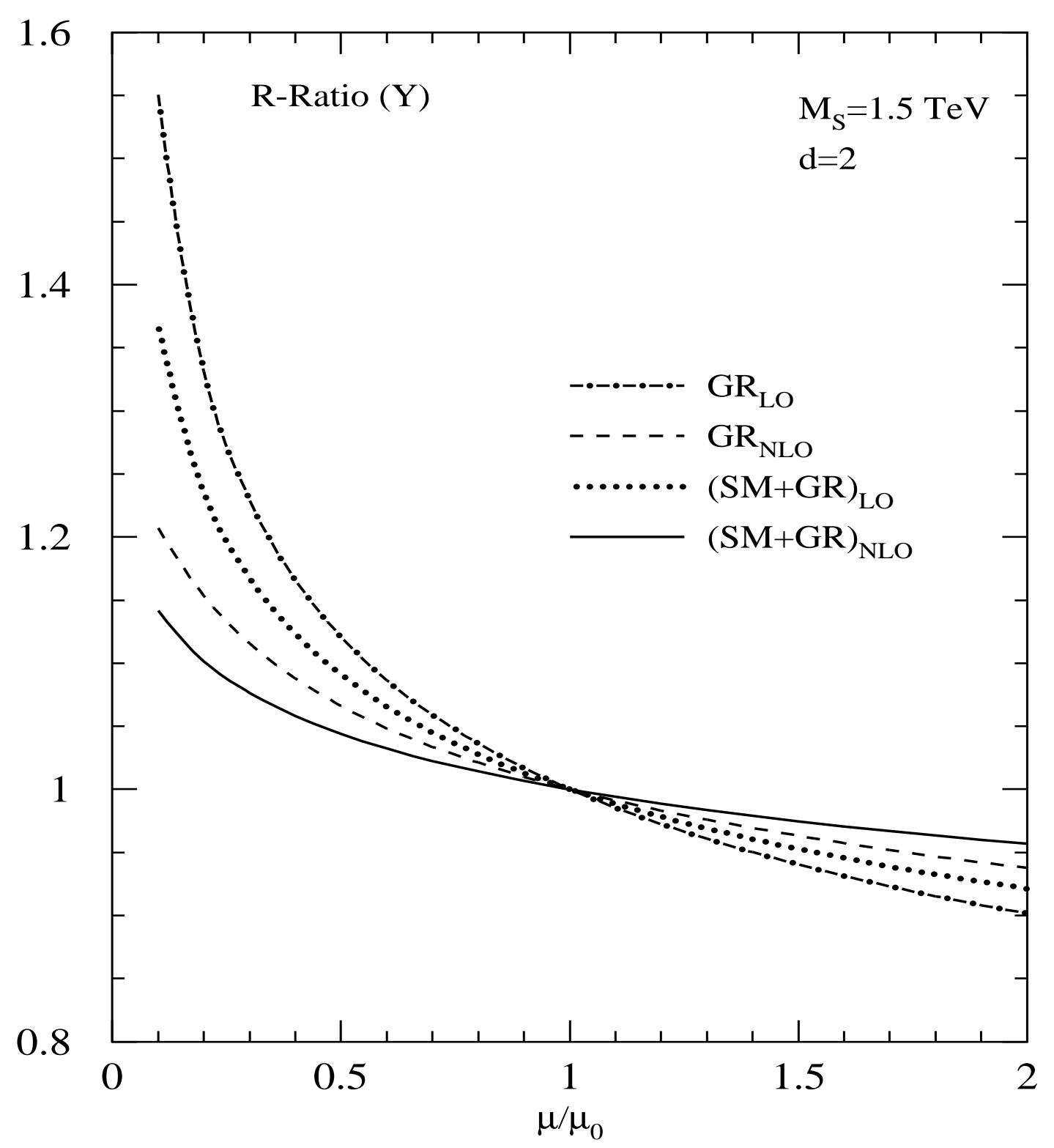

Fig. 15c 


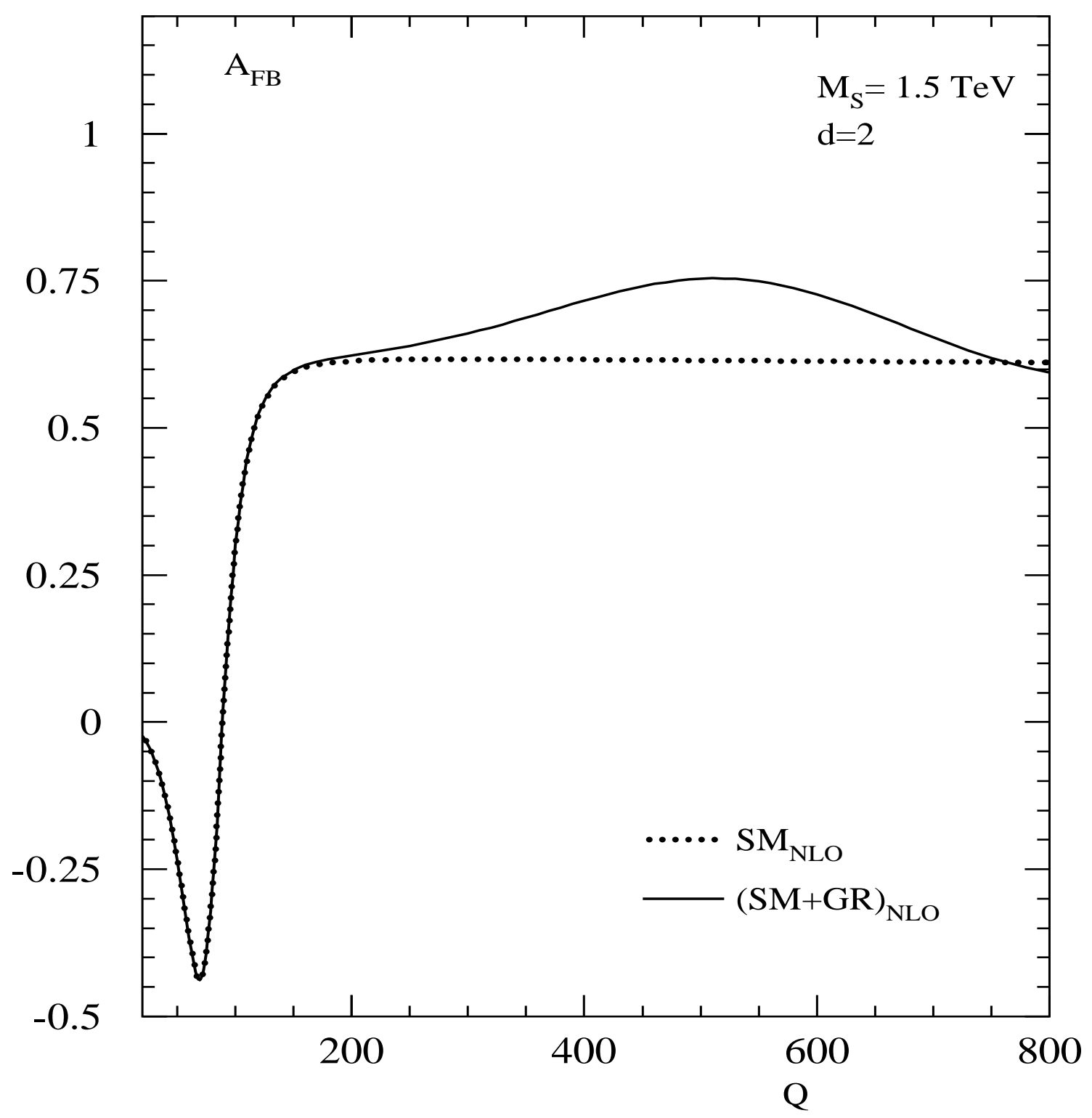

Fig. 16 